\title{
FINITE GAP JACOBI MATRICES, I. THE ISOSPECTRAL TORUS
}

\author{
JACOB S. CHRISTIANSEN*, BARRY SIMON*, , AND MAXIM ZINCHENKO*
}

\begin{abstract}
Let $\mathfrak{e} \subset \mathbb{R}$ be a finite union of disjoint closed intervals. In the study of OPRL with measures whose essential support is $\mathfrak{e}$, a fundamental role is played by the isospectral torus. In this paper, we use a covering map formalism to define and study this isospectral torus. Our goal is to make a coherent presentation of properties and bounds for this special class as a tool for ourselves and others to study perturbations. One important result is the expression of Jost functions for the torus in terms of theta functions.
\end{abstract}

\section{INTRODUCTION}

Let $\mathfrak{e} \subset \mathbb{R}$ be a union of $\ell+1$ disjoint closed intervals

$$
\begin{aligned}
& \mathfrak{e}=\mathfrak{e}_{1} \cup \mathfrak{e}_{2} \cup \cdots \cup \mathfrak{e}_{\ell+1} \\
& \mathfrak{e}_{j}=\left[\alpha_{j}, \beta_{j}\right] \\
& \alpha_{1}<\beta_{1}<\alpha_{2}<\cdots<\alpha_{\ell+1}<\beta_{\ell+1}
\end{aligned}
$$

$\ell$ counts the number of gaps.

For later purposes, we will need to exploit potential theoretic objects associated to $\mathfrak{e}$. $\operatorname{cap}(\mathfrak{e})$ will be its logarithmic capacity, $d \rho_{\mathfrak{e}}$ the equilibrium measure (normalized by $\rho_{\mathfrak{e}}(\mathbb{R})=1$ )

$$
d \rho_{\mathfrak{e}}(x)=\rho_{\mathfrak{e}}(x) d x
$$

and $\rho_{\mathfrak{e}}\left(\mathfrak{e}_{j}\right)$ the harmonic measures. For reasons that become clear soon, we say $\mathfrak{e}$ is periodic if all harmonic measures, $\rho_{\mathfrak{e}}\left(\mathfrak{e}_{j}\right), j=1, \ldots, \ell+1$, are rational. See [34, 40, 54, 61, 68, 73] for discussions of potential theory.

Date: September 25, 2008.

2000 Mathematics Subject Classification. 42C05, 58J53, $14 \mathrm{H} 30$.

Key words and phrases. Isospectral torus, covering map, orthogonal polynomials.

* Mathematics 253-37, California Institute of Technology, Pasadena, CA 91125.

E-mail: stordal@caltech.edu; bsimon@caltech.edu; maxim@caltech.edu.

$\dagger$ Supported in part by NSF grant DMS-0652919. 
We will be interested in one- and two-sided Jacobi matrices: onesided with parameters labelled $\left\{a_{n}, b_{n}\right\}_{n=1}^{\infty}$,

$$
J=\left(\begin{array}{ccccc}
b_{1} & a_{1} & 0 & 0 & \ldots \\
a_{1} & b_{2} & a_{2} & 0 & \ldots \\
0 & a_{2} & b_{3} & a_{3} & \ldots \\
\vdots & \vdots & \vdots & \vdots & \ddots
\end{array}\right)
$$

and two-sided with $\left\{a_{n}, b_{n}\right\}_{n=-\infty}^{\infty}$ extended to the top and left in the obvious way. And, of course, we want to consider the orthogonal polynomials on the real line (OPRL) [27, 65, 70] defined by

$$
\begin{gathered}
p_{-1}(x)=0 \quad p_{0}(x)=1 \\
x p_{n}(x)=a_{n+1} p_{n+1}(x)+b_{n+1} p_{n}(x)+a_{n} p_{n-1}(x)
\end{gathered}
$$

If $d \mu$ is the spectral measure for $J$ and vector $(1,0,0, \ldots)^{t}$, then the $p_{n}$ 's are orthonormal

$$
\int p_{n}(x) p_{m}(x) d \mu(x)=\delta_{n m}
$$

We will also want to consider monic OPs, $P_{n}$, the multiple of $p_{n}$ with leading coefficient 1 ,

$$
\begin{aligned}
p_{n}(x) & =\left(a_{1} \cdots a_{n}\right)^{-1} P_{n}(x) \\
x P_{n}(x) & =P_{n+1}(x)+b_{n+1} P_{n}(x)+a_{n}^{2} P_{n-1}(x)
\end{aligned}
$$

We want to analyze the case where

$$
\sigma_{\mathrm{ess}}(J) \equiv \sigma_{\mathrm{ess}}(d \mu)=\mathfrak{e}
$$

Here $\sigma_{\text {ess }}(J)$ is the essential spectrum of $J$, aka the derived set of $\operatorname{supp}(d \mu)$. We will use $\sigma(J)$ (or $\sigma(d \mu)$ ) for the spectrum of $J$ and $\Sigma_{\mathrm{ac}}(d \mu)=\left\{x \mid \frac{d \mu}{d x} \neq 0\right\}$ for the essential support of the a.c. part of $d \mu$. In this paper, we will focus on the isospectral torus, in [15] on the Szegö class, and in [16] on results that go beyond the Szegö class. Some of our results were announced in [14].

The goal is to extend what is known about the case $\mathfrak{e}=[-1,1]$. This can be viewed as a problem in approximation theory where polynomial asymptotics is critical or as a problem in spectral theory where Jacobi parameter asymptotics is critical. As usual, there are three main levels from the point of view of polynomial asymptotics:

(a) Root asymptotics. Asymptotics of $\left|P_{n}(x)\right|^{1 / n}$. For $[-1,1]$, the theory is due to Erdös-Turán [22] and Ullman [74, 75, 76]. For general sets, including finite gap sets, the theory is due to Stahl-Totik [68] (see 
Simon [61] for a review). One has for $x \notin \sigma(d \mu)$ and $d \mu$ regular (i.e., $\sigma_{\text {ess }}(d \mu)=\mathfrak{e}$ and $\left.\lim \left(a_{1} \cdots a_{n}\right)^{1 / n}=\operatorname{cap}(\mathfrak{e})\right)$ that

$$
\left|P_{n}(x)\right|^{1 / n} \rightarrow \exp \left(\int \log |x-y| d \rho_{\mathfrak{e}}(y)\right)
$$

(b) Ratio asymptotics. Traditionally, this involves the ratio $P_{n+1}(x) / P_{n}(x)$ having a limit. Nevai [47] showed that if $a_{n} \rightarrow a$, $b_{n} \rightarrow b(a \neq 0)$ so that $\sigma_{\mathrm{ess}}(d \mu)=[b-2 a, b+2 a]$, then the limit exists for $x \notin \sigma(d \mu)$. Simon [58] proved a converse: if the limit exists at a single point in $\mathbb{C}_{+}=\{z \mid \operatorname{Im} z>0\}$, then for some $a, b$, we have that $a_{n} \rightarrow a, b_{n} \rightarrow b$. Thus, the proper analog for $\sigma_{\text {ess }}(d \mu)=\mathfrak{e}$ will not be existence of a limit but something more subtle. This is an interesting open question which we will not address.

(c) Szegö asymptotics. This says that for $z \notin \sigma(d \mu)$, $P_{n}(z) / D(z) E(z)^{n} \rightarrow 1$ for an explicit function $E\left(\left(z+\sqrt{z^{2}-1}\right)\right.$ for $\mathfrak{e}=[-1,1])$ and a function $D$ which is $\mu$-dependent. The proper analog for general finite gap sets was obtained by Widom [81] (see also Aptekarev [3]) and by Peherstorfer-Yuditskii [50] using variational methods. The ratio is only asymptotically (almost) periodic. One of our main goals in this series is to provide a new nonvariational approach to this result. In addition, following Damanik-Simon [19] for $[-1,1]$, we want to consider cases where the Szego" condition fails.

From the spectral theory point of view, the analogs of $a_{n} \rightarrow \frac{1}{2}$, $b_{n} \rightarrow 0$ (aka the Nevai class) concern the isospectral torus, an object we will discuss extensively in this paper. For now, we note that if $\mathfrak{e}$ is periodic, the $J$ 's in the isospectral torus are all periodic Jacobi matrices with $\sigma_{\text {ess }}(J)=\mathfrak{e}$. In the general case, it is an $\ell$-dimensional torus of almost periodic $J$ 's with $\sigma_{\text {ess }}(J)=\mathfrak{e}$. It can be singled out via minimal Herglotz functions [65] or reflectionless potentials [55]; see Section 6 below.

The key realization is that the Nevai class needs to be replaced by approach to an isospectral torus. This was first noted by Simon [59, 60] as conjectures in the context of the OPUC case. In turn, Simon was motivated by work of López and collaborators [6, 9] who studied the case of a single gap for OPUC.

From a spectral point of view, the analogs of the asymptotics results are:

(a) Regularity implies more restrictions on the Jacobi parameters than $\left(a_{1} \cdots a_{n}\right)^{1 / n} \rightarrow \operatorname{cap}(\mathfrak{e})$. For example, for $\mathfrak{e}=[-1,1]$, it is known that $\frac{1}{n} \sum_{j=1}^{n}\left(a_{j}-\frac{1}{2}\right)^{2}+b_{j}^{2} \rightarrow 0$ and, for $\mathfrak{e}$ periodic, a similar Cesàro convergence result for distances to the isospectral torus is proven in 
63. The analog for general finite gap sets remains an interesting open question.

(b) The key result here in the case $\mathfrak{e}=[-1,1]$ is the theorem of Denisov-Rakhmanov [20] stating that if $\Sigma_{\mathrm{ac}}(d \mu)=\sigma_{\mathrm{ess}}(d \mu)=[-1,1]$, then $a_{n} \rightarrow \frac{1}{2}, b_{n} \rightarrow 0$. Simon [60] conjectured that for periodic $\mathfrak{e}$, the proper result is that if $\Sigma_{\mathrm{ac}}(d \mu)=\sigma_{\mathrm{ess}}(d \mu)=\mathfrak{e}$, then all right limits lie in the isospectral torus. For periodic $\mathfrak{e}$, this was proven by DamanikKillip-Simon [18] who conjectured the result for general $\mathfrak{e}$. It was then proven for general finite gap sets by Remling [55]. Remling's result plays a key role in our work in paper II [15]. We note that in the opposite direction, Last-Simon [41] have shown that if all right limits lie in the isospectral torus, then $\sigma_{\text {ess }}(d \mu)=\mathfrak{e}$.

(c) Here there are two main results. When $\sigma(d \mu)=\mathfrak{e}$ (no bound states), Widom proved that a Szegö condition implies

$$
\begin{gathered}
\liminf \frac{a_{1} \cdots a_{n}}{\operatorname{cap}(\mathfrak{e})^{n}}>0 \\
\limsup \frac{a_{1} \cdots a_{n}}{\operatorname{cap}(\mathfrak{e})^{n}}<\infty
\end{gathered}
$$

The Szegö condition in this situation is

$$
\int_{\mathfrak{e}} \operatorname{dist}(x, \mathbb{R} \backslash \mathfrak{e})^{-1 / 2} \log \left(\frac{d \mu}{d x}\right) d x>-\infty
$$

Widom allowed no eigenvalues outside $\mathfrak{e}$. Peherstorfer-Yuditskii [50] had eigenvalues, but only in a later note [51] did they have the natural (from their paper [49]) condition

$$
\sum_{j} \operatorname{dist}\left(x_{j}, \mathfrak{e}\right)^{1 / 2}<\infty
$$

where $x_{j}$ are the point masses of $d \mu$ (or eigenvalues of $J$ ) outside $\mathfrak{e}$. Thus, Peherstorfer-Yuditskii [51] showed

$$
1.14+1.15 \Rightarrow 1.12+1.13
$$

One of our main results in paper II [15] is to show

$$
1.12+1.15 \Rightarrow 1.14+1.13
$$

Peherstorfer remarked to us that, while this result is new, it can also be derived from the results of [50].

The key to our analysis is a machinery developed by Sodin-Yuditskii [66] and exploited by Peherstorfer-Yuditskii [50, 51]. To explain it, we 
note that the key to recent sum rule discussions (summarized in [65]) is to take the $m$-function given by

$$
m(z)=\int \frac{d \mu(x)}{x-z}
$$

and in the case $\mathfrak{e}=[-2,2]$, move it to $\mathbb{D}=\{z|| z \mid<1\}$ via

$$
M(z)=-m\left(z+z^{-1}\right)
$$

The map

$$
\mathbf{x}(z)=z+z^{-1}
$$

is the unique analytic bijection of $\mathbb{D}$ to $\mathbb{C} \cup\{\infty\} \backslash[-2,2]$ with

$$
\mathbf{x}(0)=\infty \quad \lim _{\substack{z \rightarrow 0 \\ z \neq 0}} z \mathbf{x}(z)>0
$$

The minus sign in 1.19 comes from the fact that $\mathbf{x}$ maps $\mathbb{D} \cap \mathbb{C}_{+}$to $-\mathbb{C}_{+}$(where $\mathbb{C}_{+}=\{z \mid \operatorname{Im} z>0\}$ ).

In our case, there cannot be an analytic bijection of $\mathbb{D}$ to $\mathbb{C} \cup\{\infty\} \backslash \mathfrak{e}$ since $\mathbb{C} \cup\{\infty\} \backslash \mathfrak{e}$ is not simply connected. However, because the holomorphic universal cover of $\mathbb{C} \cup\{\infty\} \backslash \mathfrak{e}$ is $\mathbb{D}$, there is an analytic map $\mathbf{x}: \mathbb{D} \rightarrow \mathbb{C} \cup\{\infty\} \backslash \mathfrak{e}$ which is locally one-one and obeys (1.21). Moreover, there is a group $\Gamma$ of Möbius transformations of $\mathbb{D}$ to $\mathbb{D}$ so that

$$
\mathbf{x}(z)=\mathbf{x}(w) \Leftrightarrow \exists \gamma \in \Gamma \text { so that } z=\gamma(w)
$$

This group is isomorphic to $\pi_{1}(\mathbb{C} \cup\{\infty\} \backslash \mathfrak{e})=\mathbb{F}_{\ell}$, the free nonabelian group on $\ell$ generators. We mention that $\mathbf{x}$ is uniquely determined if (1.21) - 1.22 hold and $\mathbf{x}$ is locally one-one.

Our goal in this paper is to discuss the isospectral torus in terms of this formalism. It turns out that basic objects for the isospectral torus, like Bloch waves and Green's function behavior, are not discussed in detail anywhere. We will remedy that here. While these results will not be surprising to experts, they are exceedingly useful both in our further works [15, 16] and in [10, 26, 35, 64].

We should expand on the point we already remarked upon that there are two distinct ways of describing the isospectral torus: as a set of minimal Herglotz functions or as the family of reflectionless Jacobi matrices with spectrum $\mathfrak{e}$. The view as minimal Herglotz functions goes back to the earliest periodic KdV work [21, 44] (see also [24, 39, 78]), while the reflectionless definition goes back at least to Sodin-Yuditskii [66] (see also [55]).

There is an important distinction: reflectionless objects are natural whole-line (doubly infinite) Jacobi matrices, while minimal Herglotz functions are associated to half-line objects. Of course, the passage 
from whole-line to half-line objects is by restriction-but the converse is not so simple. From our point of view, the key is that the $J$ 's associated to minimal Herglotz functions are quasiperiodic and such functions are determined by their values on a half-line (because a quasiperiodic function vanishing on a half-line is identically zero). Alternatively, if $m(z)$ is a minimal Herglotz function, the demand that

$$
m_{0}(z)=\mathcal{M}\left(a_{0}, b_{0}, m(z)\right)
$$

where

$$
\mathcal{M}(a, b, f(z))=\frac{1}{-z+b-a^{2} f(z)}
$$

be a minimal Herglotz function determines $a_{0}$ and $b_{0}$, and so inductively, minimality allows a unique continuation from the half-line.

In Section 2, we describe the map $\mathbf{x}$ in (1.21) and its natural extension to a covering (albeit not universal covering) map of the two-sheeted Riemann surface, $\mathcal{S}$, that the $m$-function for elements of the isospectral torus lives on. In Section 3, we describe a critical result of Beardon [8] on the Poincaré index of $\Gamma$. Section 4 reviews the facts about character automorphic Blaschke products and their connection to potential theory. We will also present estimates on these products needed in later papers [15, 16]. In Section 5, we use this machinery to prove Abel's theorem. In Section 6, we describe the isospectral torus as the family of minimal Herglotz functions on $\mathcal{S}$. Sections 7 and 8 will describe the Jost functions of elements of the isospectral torus and will prove that the natural map from the isospectral torus to the group of characters of $\Gamma$, given by the character of the Jost function, is an isomorphism of tori. We will also relate Jost functions to theta functions, one of the more significant results of the present paper. Section 9 will discuss Jost solutions and the associated Bloch waves. Finally, Section 10 will apply these solutions to the study of the Green's function. Some of the material in Sections 2, 4, and 6 is in suitable texts but included here because we wish to make this paper more accessible to approximation theorists who may be unfamiliar with it.

We also mention the enormous debt this paper owes to the seminal work of Sodin-Yuditskii [66] and Peherstorfer-Yuditskii [50]. About the only real advantage of our presentation in this first paper over ideas implicit in [66, 50] is that we are more explicit and our Jost functions, unlike the close relatives in [66, 50], are strictly character automorphic. We emphasize that [66, 50] had as their focus the theory of certain infinite gap sets for which $\mathfrak{e}$ is typically a Cantor set of positive Lebesgue measure. But they include finite gap sets and provide useful 
tools in that special case. Our work makes use of some results special to this finite gap situation.

We note that while we discuss Jost functions and solutions here for the isospectral torus, in [15, 16] we will present them for any $J$ in the Szegö class. For us, they are the key to understanding Szegő asymptotics in this finite gap situation.

We want to thank D. Calegari, H. Farkas, F. Gesztesy, I. Kra, N. Makarov, F. Peherstorfer, and P. Yuditskii.

\section{The Covering Map and the Fuchsian Group}

In this section, we describe the basic objects and setup that we will use. We emphasize that these constructs are not new here, and more than anything else, this section sets up notation and gives a pedagogical introduction. The Riemann surface, $\mathcal{S}$, was introduced for finite gap $\mathrm{KdV}$ in [21, 44] and for finite gap Jacobi matrices in [24, 39, 78]. The Fuchsian group formalism is from [66].

Let $\mathcal{S}_{+}$be the set $\mathbb{C} \cup\{\infty\} \backslash \mathfrak{e}$ viewed as a Riemann surface. First of all, we want to view this as one sheet of the Riemann surface of the function

$$
w=(R(z))^{1 / 2}
$$

where

$$
R(z)=\prod_{j=1}^{\ell+1}\left(z-\alpha_{j}\right)\left(z-\beta_{j}\right)
$$

More explicitly, we consider pairs $(w, z)$ in $\mathbb{C}^{2}$ obeying

$$
w^{2}-R(z) \equiv G(w, z)=0
$$

Since $\frac{\partial G}{\partial z} \neq 0$ at those $2 \ell+2$ points where $\frac{\partial G}{\partial w}=0$, this set is a onedimensional complex manifold, aka a Riemann surface.

With two points at infinity added, this set becomes a compact surface $\mathcal{S}$. One can formally define $\mathcal{S}$ by looking in $\mathbb{C}^{3} \backslash\{0\}$ at triples, $(w, z, u)$, with

$$
w^{2} u^{2 \ell}=\prod_{j=1}^{\ell+1}\left(z-\alpha_{j} u\right)\left(z-\beta_{j} u\right)
$$

and regarding $(w, z, u)$ as equivalent to $\left(w^{\prime}, z^{\prime}, u^{\prime}\right)$ if there is $\lambda \in \mathbb{C} \backslash\{0\}$, so $w=\lambda w^{\prime}, z=\lambda z^{\prime}, u=\lambda u^{\prime}$. Rather than this formal projective space view, we will think of two points $\infty_{ \pm} \in \mathcal{S}$, obtained by using $\zeta=1 / z$ coordinates on $\mathcal{S}_{ \pm}$and adding the missing point $\zeta=0$.

There is a natural map $\pi: \mathcal{S} \rightarrow \mathbb{C} \cup\{\infty\}$ given by $(w, z) \rightarrow z$. It sets up $\mathcal{S}$ as a branched cover of $\mathbb{C} \cup\{\infty\} . \pi$ is two-one on all points in $\mathbb{C} \cup\{\infty\}$ except $\left\{\alpha_{j}, \beta_{j}\right\}_{j=1}^{\ell+1}$ - these latter points are the branch points. 
There is a second natural map $\tau: \mathcal{S} \rightarrow \mathcal{S}$ that in $(w, z)$ coordinates takes $w \rightarrow-w$. $\mathcal{S}_{-}$will denote the image of $\mathcal{S}_{+}$under $\tau . \tau\left(\infty_{+}\right)=\infty_{-}$. $\mathcal{S} \backslash\left(\mathcal{S}_{+} \cup \mathcal{S}_{-}\right)$is thus $\pi^{-1}(\mathfrak{e})$. Each $\pi^{-1}\left(\mathfrak{e}_{j}\right)$ is topologically a circle.

There is a close connection between $\mathcal{S}$ and the potential theory associated to $\mathfrak{e}$. In terms of the equilibrium measure, $d \rho_{\mathfrak{e}}$, consider its Borel transform,

$$
M_{\mathfrak{e}}(z)=\int \frac{d \rho_{\mathfrak{e}}(x)}{x-z}
$$

It is a basic fact (due to Craig [17]; see also [61, 65]) that for suitable points, $x_{j} \in\left(\beta_{j}, \alpha_{j+1}\right)$, we have

$$
M_{\mathfrak{e}}(z)=\frac{-\prod_{j=1}^{\ell}\left(z-x_{j}\right)}{\left(\prod_{j=1}^{\ell+1}\left(z-\alpha_{j}\right)\left(z-\beta_{j}\right)\right)^{1 / 2}}
$$

so $M_{\mathfrak{e}}$ has a natural analytic continuation from $\mathbb{C} \cup\{\infty\} \backslash \mathfrak{e}$ to $\mathcal{S}$.

Topologically, $\mathcal{S}$ is the sphere with $\ell$ handles attached - the canonical surface of genus $\ell$. Its first homology group (see [4, 32, 79] for basic topological notions we use here) is $\mathbb{Z}^{2 \ell}$. One way of looking at the generators is picking curves that loop around each $\pi^{-1}\left(\mathfrak{e}_{j}\right)$ but one (the sum of all $\ell+1$ is homologous to zero) and also curves that loop around each

$$
\pi^{-1}\left(\left[\beta_{j}, \alpha_{j+1}\right]\right) \equiv G_{j} \quad j=1, \ldots, \ell
$$

For a while, we put $\mathcal{S}$ aside and focus on $\mathcal{S}_{+} . \mathcal{S}_{+}$is not simply connected. Its fundamental group is the free nonabelian group on $\ell$ generators. We will pick $\infty$ as the base point. One way of picking generators is to pick $\tilde{\gamma}_{1}, \ldots, \tilde{\gamma}_{\ell}$ where $\tilde{\gamma}_{j}$ is the curve that starts at $\infty$, traverses in $\mathbb{C}_{-}$to $\frac{1}{2}\left(\beta_{j}+\alpha_{j+1}\right)$ (in the $j$-th gap), and returns to $\infty$ in $\mathbb{C}_{+}$(see the lower half of Fig. 2 below).

The universal cover of $\mathcal{S}_{+}$inherits the local complex structure of $\mathcal{S}_{+}$ and so is a Riemann surface. The deck transformations preserve this complex structure so there is a discrete group, $\Gamma$, of complex automorphisms of the universal cover where each $\gamma \in \Gamma$ has no fixed points.

It is a fundamental result in the theory of Riemann surfaces (the uniformization theorem; see [23, 30, 46]) that the only simply connected Riemann surfaces are the Riemann sphere, the complex plane, and the unit disk, $\mathbb{D}$. The sphere has no fixed point free complex automorphism and the only discrete groups of automorphisms on $\mathbb{C}$ are one- and twodimensional lattices, so the only Riemann surfaces with cover $\mathbb{C}$ are the tori and the punctured disk. Since $\mathcal{S}_{+}$is neither of these, its universal cover is $\mathbb{D}$. 
Thus, there exists a map $\mathbf{x}: \mathbb{D} \rightarrow \mathcal{S}_{+}$, which is locally one-one, and a group, $\Gamma$, of Möbius transformations on $\mathbb{D}$ so that $(1.22$ holds. By requiring (1.21) (which, by the action of maps on $\mathbb{D}$, we can always do), we uniquely fix $\mathbf{x}$. There is a lovely proof of the existence of $\mathbf{x}$ due to Radó [53] that follows the standard proof of the Riemann mapping theorem ([1]); see [65, Sect. 9.5].

$\Gamma$ is a discrete group of Möbius transformations leaving $\mathbb{D}$ setwise fixed, aka a Fuchsian group. For background on such groups, see [37] or [65, Ch. 9].

$\mathcal{S}_{+}$is invariant under complex conjugation, as is $\mathbb{D}$, so $\overline{\mathbf{x}(\bar{z})}$ is also a covering map of $\mathbb{D}$ over $\mathcal{S}_{+}$. But it obeys 1.21 , so by uniqueness,

$$
\mathbf{x}(\bar{z})=\overline{\mathbf{x}(z)}
$$

We define the fundamental region, $\mathcal{F}^{\text {int }} \subset \mathbb{D}$, as follows: $\mathbf{x}^{-1}(\mathbb{C} \cup$ $\left.\{\infty\} \backslash\left[\alpha_{1}, \beta_{\ell+1}\right]\right)$ consists of connected components on which $\mathbf{x}$ is a bijection (this is because $\mathbb{C} \cup\{\infty\} \backslash\left[\alpha_{1}, \beta_{\ell+1}\right]$ is simply connected, and so contains no closed curve nonhomotopic to the trivial curve in $\mathcal{S}_{+}$). We let $\mathcal{F}^{\text {int }}$ be the component containing $0 \in \mathbf{x}^{-1}(\{\infty\})$; we will shortly enlarge $\mathcal{F}^{\text {int }}$ to a fundamental set, $\mathcal{F}$.

In $\mathcal{F}^{\text {int }}$, consider $\mathbf{x}^{-1}\left(\mathbb{R} \cup\{\infty\} \backslash\left[\alpha_{1}, \beta_{\ell+1}\right]\right)$. By (2.8), the set is a subset of $\mathbb{D} \cap \mathbb{R}$. But as $y \rightarrow \alpha_{1}$ or $\beta_{\ell+1}, \mathbf{x}^{-1}(y)$ must approach the boundary of $\mathbb{D}$. It follows that $\mathbf{x}^{-1}\left(\mathbb{R} \cup\{\infty\} \backslash\left[\alpha_{1}, \beta_{\ell+1}\right]\right)=(-1,1) \subset \mathbb{D}$. The other inverse images of this set are, by 1.22 , images of $(-1,1)$ under Möbius transformations, so arcs of orthocircles, that is, pieces of circles orthogonal to $\partial \mathbb{D}$.

In place of 1.21 , we could have required $\mathbf{x}(0)=\frac{1}{2}\left(\beta_{j}+\alpha_{j+1}\right)$ together with $\mathbf{x}^{\prime}(0)>0$ and seen that for this $\mathbf{x}$, one has $(-1,1)$ in the inverse image of the gap $\left(\beta_{j}, \alpha_{j+1}\right)$. Since the two $\mathbf{x}$ 's are related by a Möbius transformation, we conclude that under our $\mathbf{x}$ (normalized by (1.21) the inverse images of gaps are also arcs of orthocircles. The boundary of $\mathcal{F}^{\text {int }}$ in $\mathbb{D}$ (not $\overline{\mathbb{D}}$ ) clearly has $2 \ell$ pieces corresponding to the tops and bottoms of the $\ell$ gaps. Thus, $\mathcal{F}^{\text {int }}$ is $\mathbb{D}$ with $2 \ell$ orthocircles (and their interior) removed - $\ell$ in each half-plane - these are conjugate to one another. We label the boundary pieces in the upper half-disk $C_{1}^{+}, \ldots, C_{\ell}^{+}$. Figure 1 shows a typical $\mathcal{F}^{\text {int }}$ for $\ell=2$ with the inverse image of $\mathbb{C}_{-} \cap \mathcal{S}_{+}$shaded.

We now define a fundamental set, $\mathcal{F}$, by adding the $\operatorname{arcs} C_{1}^{+}, \ldots, C_{\ell}^{+}$ to $\mathcal{F}^{\text {int }}$. With this definition, every point in $\mathbb{D}$ can be uniquely written as $\gamma(w)$ for some $w \in \mathcal{F}$ and some $\gamma \in \Gamma$. The fundamental region $\mathcal{F}^{\text {int }}$ is indeed the interior of $\mathcal{F}$. As a subset of $\mathbb{D}, \overline{\mathcal{F}}$ has $C_{1}^{-}, \ldots, C_{\ell}^{-}$added. 


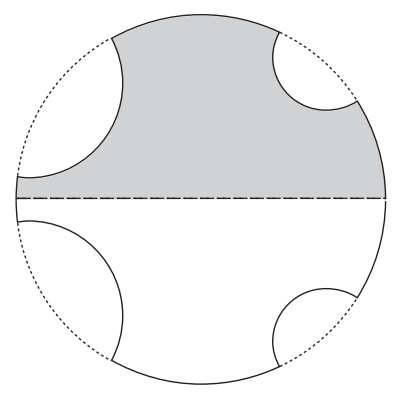

Figure 1. The fundamental region

Here $C_{j}^{-}$denotes the complex conjugate of $C_{j}^{+}$. Sometimes we want to consider the closure of $\mathcal{F}$ in $\overline{\mathbb{D}}$, that is, also add the $2 \ell$ arcs in $\partial \mathbb{D}$ at the ends.

To describe the Fuchsian group, $\Gamma$, we begin with the $\ell$ generators: the deck transformations that go into the generators, $\tilde{\gamma}_{1}, \ldots, \tilde{\gamma}_{\ell}$, of the homotopy group, $\pi_{1}\left(\mathcal{S}_{+}\right)$. Figure 2 shows the lift of the curve associated to $\tilde{\gamma}_{2}$ in our example. The bottom half of the curve in $\mathcal{S}_{+}$under $\mathbf{x}^{-1}$ goes from 0 to a point on $C_{2}^{+}$. Since that half of $\tilde{\gamma}_{2}$ lies in $\mathbb{C}_{-}$, this piece of curve lies in $\mathbb{C}_{+} \cap \mathcal{F}$. The second half must be the inversion in the curve $C_{2}^{+}$of the first half, and so it is as shown.

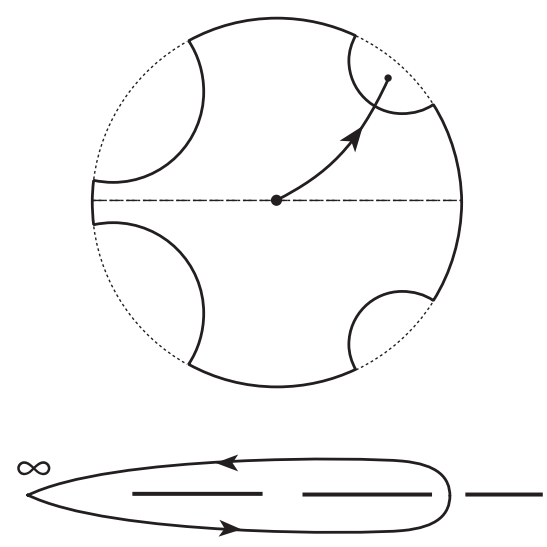

FiguRE 2. Fuchsian group generators

A little thought shows that the Möbius map that corresponds to $\tilde{\gamma}_{2}$, which we will call $\gamma_{2}$, is what one gets by composing complex conjugation with inversion in $C_{2}^{+}$. Inversion in the circle $\left|z-z_{0}\right|=r$ is the 
map

$$
z \rightarrow z_{0}+\frac{r^{2}}{\bar{z}-\bar{z}_{0}}
$$

Thus,

$$
\gamma_{j}=r_{j}^{+} c
$$

where $c(z)=\bar{z}$ and $r_{j}^{+}$is inversion in $C_{j}^{+}$.

$\Gamma$ is the free nonabelian group generated by $\left\{\gamma_{j}\right\}_{j=1}^{\ell}$. Every element of $\Gamma$ can be uniquely written as $\alpha_{w(\gamma)} \cdots \alpha_{2} \alpha_{1}$ where each $\alpha_{j}$ is either a $\gamma_{j}$ or a $\gamma_{j}^{-1}$ and no $\alpha_{j}$ is an $\alpha_{j-1}^{-1} . w(\gamma)$ is the word length of $\gamma$. It will be convenient to define

$$
\Gamma_{k}=\{\gamma \mid w(\gamma)=k\}
$$

We have $\# \Gamma_{k}=2 \ell(2 \ell-1)^{k-1}$ since $\alpha_{1}$ has $2 \ell$ choices $\left(\gamma_{1}, \ldots, \gamma_{\ell}\right.$, $\left.\gamma_{1}^{-1}, \ldots, \gamma_{\ell}^{-1}\right)$ and each other $\alpha_{j}$ has $2 \ell-1$ choices. By definition, $\Gamma_{0}=\{1\}$.

Alternatively, one can write for $\gamma \in \Gamma_{2 m}$,

$$
\gamma=s_{1} \cdots s_{2 m}
$$

with each $s_{k}$ an $r_{j}^{ \pm}\left(r_{j}^{-}\right.$is inversion in $\left.C_{j}^{-}\right)$, and for $\gamma \in \Gamma_{2 m+1}$,

$$
\gamma=s_{1} \cdots s_{2 m+1} c
$$

We point out that $\mathcal{F}$ is the Dirichlet fundamental region for $\Gamma$, that is,

$$
\overline{\mathcal{F}}=\{z|| \gamma(z)|\geq| z \mid \text { for all } \gamma \in \Gamma\}
$$

Moreover, $C_{j}^{+}$is the perpendicular bisector in the hyperbolic metric of 0 and $\gamma_{j}(0)$ (see, e.g., [65, Sect. 9.3]).

Since $\gamma \in \Gamma$ has no fixed points in $\mathbb{D}$, it cannot be elliptic, and it is not hard to see [65] that it is, in fact, hyperbolic.

The fact that $\mathcal{F}$ is a fundamental set implies that

$$
\mathbb{D}=\bigcup_{\gamma \in \Gamma} \gamma(\mathcal{F})
$$

We will let

$$
\mathbb{D}_{k}=\bigcup_{w(\gamma) \leq k} \gamma(\mathcal{F})
$$

and

$$
\mathcal{R}_{k}=\mathbb{D} \backslash \mathbb{D}_{k}
$$

and finally define $\partial \mathcal{R}_{k} \subset \partial \mathbb{D}$ as

$$
\partial \mathcal{R}_{k}=\overline{\mathcal{R}}_{k} \cap \partial \mathbb{D}
$$

where the closure is taken in $\overline{\mathbb{D}}$. Thus, $\mathbb{D}_{k}$ is connected, while $\mathcal{R}_{k}$ consists of $2 \ell(2 \ell-1)^{k}$ disks (intersected with $\mathbb{D}$ ) with only some boundaries 
included and $\partial \mathcal{R}_{k}$ is $2 \ell(2 \ell-1)^{k}$ connected arcs in $\partial \mathbb{D}$. Figure 3 shows the $\operatorname{arcs} C_{j}^{ \pm}$and their images under $\Gamma_{1}$ and $\Gamma_{2}$.

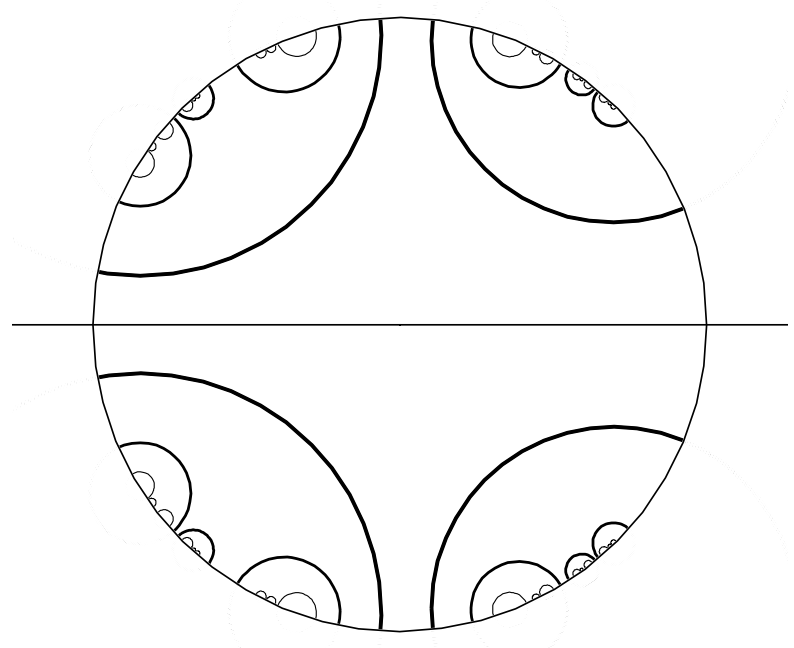

FiguRE 3. Iterated generators

In Figure 3, the union of the large partial disks is $\mathcal{R}_{0}$, the union of the medium partial disks, $\mathcal{R}_{1}$, and the union of the tiny partial disks, $\mathcal{R}_{2}$.

Notice the geometry is such that

$$
z \in \mathcal{R}_{k} \Rightarrow \frac{z}{|z|} \in \partial \mathcal{R}_{k}
$$

which we will need in Section 7 and [15].

We denote by $\mathcal{L}$ the set of limit points of $\Gamma$. It is a subset of $\partial \mathbb{D}$ and can be defined via several equivalent definitions:

(i) $\mathcal{L}=\cap_{k} \overline{\mathcal{R}}_{k}$

(ii) $\mathcal{L}=\overline{\{z \in \partial \mathbb{D} \mid \gamma(z)=z \text { for some } \gamma \neq 1 ; \gamma \in \Gamma\}}$

(iii) $\mathcal{L}=\overline{\{\gamma(0) \mid \gamma \in \Gamma\}} \cap \partial \mathbb{D}$.

In (ii), each $\gamma \in \Gamma$ is hyperbolic, so it has two fixed points on $\partial \mathbb{D}$, each of which is either $\lim _{n \rightarrow \infty} \gamma^{n}(0)$ or $\lim _{n \rightarrow \infty} \gamma^{-n}(0)$. This is the key to proving that (ii) and (iii) are equivalent and that (iii) is the same if $\gamma(0)$ is replaced by $\gamma\left(z_{0}\right)$ for any fixed $z_{0} \in \mathbb{D}$. The key to understanding $(\mathrm{i})$ is that, by definition, $\mathbb{D}_{k}$ contains only finitely many $\gamma(0)$, all of which are a finite distance from $\partial \mathbb{D}$. As we will explain in the next section, $\mathcal{L}$ is of one-dimensional Lebesgue measure zero, indeed, of Hausdorff dimension strictly smaller than one. 
Now, we return to $\mathcal{S}_{+}$and the two-sheeted Riemann surface $\mathcal{S}$. The basic fact here is that the map $\mathbf{x}: \mathbb{D} \rightarrow \mathcal{S}_{+}$has an analytic continuation both to a map of $\mathbb{C} \cup\{\infty\} \backslash \mathcal{L}$ to $\mathbb{C} \cup\{\infty\}$ and to a map

$$
\mathbf{x}^{\sharp}: \mathbb{C} \cup\{\infty\} \backslash \mathcal{L} \rightarrow \mathcal{S} .
$$

By construction, $\mathbf{x}(z)$ approaches $\mathbb{R}$ as $z \rightarrow \partial \mathbb{D}$ with $z \in \mathcal{F}$. So, by the strong form of the reflection principle, $\mathbf{x}$ is continuous and real-valued up to $\partial \mathbb{D} \cap \overline{\mathcal{F}}$ and can be meromorphically continued to $(\partial \mathbb{D} \cap \overline{\mathcal{F}}) \cup \mathcal{F}^{-1}$. Utilizing 1.22 , we can thus extend $\mathbf{x}$ to a map of $\mathbb{C} \cup\{\infty\} \backslash \mathcal{L}$ onto $\mathbb{C} \cup\{\infty\}$. x outside $\overline{\mathbb{D}}$ is defined by

$$
\mathbf{x}(1 / z)=\mathbf{x}(z)
$$

At points, $z_{0}$, where $\mathbf{x}\left(z_{0}\right)$ is real and $\mathbf{x}(z)-\mathbf{x}\left(z_{0}\right)$ has a zero of order $k$, there are $2 k$ curves (asymptotically rays) coming out of $z_{0}$ on which $\mathbf{x}(z)$ is real. On $\mathbb{C}_{+} \cap \mathcal{F}, \mathbf{x}$ has negative imaginary part and so, by reflection, at points, $z_{0}$, in $\mathbb{C}_{+} \cap \partial \mathbb{D} \cap \overline{\mathcal{F}}$ - except for the endpoints, there are two rays near $z_{0}$ where $\mathbf{x}$ is real. It follows that on the set

$$
\left\{z \in \mathbb{C} \cup\{\infty\} \backslash \mathcal{L} \mid \mathbf{x}(z) \notin\left\{\alpha_{j}, \beta_{j}\right\}_{j=1}^{\ell+1}\right\}
$$

$\mathbf{x}^{\prime}$ is nonzero. At points, $z_{0}$, where $\mathbf{x}\left(z_{0}\right) \in\left\{\alpha_{j}, \beta_{j}\right\}_{j=1}^{\ell+1}$, images of $\mathbb{R}$ or a $C_{j}^{+}$under an element $\gamma \in \Gamma$ intersect $\partial \mathbb{D}$ orthogonally, so four rays on which $\mathbf{x}$ is real come out of $z_{0}$. Hence, $\mathbf{x}(z)-\mathbf{x}\left(z_{0}\right)$ has a double zero, that is, $\mathbf{x}^{\prime}\left(z_{0}\right)=0$ and $\mathbf{x}^{\prime \prime}\left(z_{0}\right) \neq 0$, and the extended map $\mathbf{x}$ is therefore not a local bijection at such points $z_{0}$. The same is true for the canonical projection $\pi: \mathcal{S} \rightarrow \mathbb{C} \cup\{\infty\}$ so if we think of $\mathrm{x}: \mathbb{D} \rightarrow \mathcal{S}_{+} \subset \mathcal{S}$ (rather than into a subset of $\mathbb{C} \cup\{\infty\}$ ), we can extend it to a map $\mathbf{x}^{\sharp}: \mathbb{C} \cup\{\infty\} \backslash \mathcal{L} \rightarrow \mathcal{S}$ via

$$
\mathbf{x}^{\sharp}(1 / z)=\tau(\mathbf{x}(z))
$$

Then the maps $\mathbf{x}$ and $\mathbf{x}^{\sharp}$ are related via

$$
\mathbf{x}=\pi \circ \mathbf{x}^{\sharp}
$$

The elements of $\Gamma$ are rational functions and so maps of $\mathbb{C} \cup\{\infty\}$ to itself. It is easy to see that each $\gamma$ maps $\mathcal{L}$ to $\mathcal{L}$ (for if $\gamma_{n}(0) \rightarrow z_{0}$, then $\left.\gamma \circ \gamma_{n}(0) \rightarrow \gamma\left(z_{0}\right)\right)$ and so $\mathbb{C} \cup\{\infty\} \backslash \mathcal{L}$ to itself. Of course, we have

$$
\mathbf{x}(\gamma(z))=\mathbf{x}(z)
$$

since $\gamma$ is analytic and this holds on $\mathbb{D}$. $\mathbf{x}^{\sharp}$ has a similar relation. Indeed, since 2.22 holds, we have 1.22 for $\mathbf{x}^{\sharp}$ on all of $\mathcal{S}$. By the unfolding discussed above, $x^{\sharp}$ is a local bijection on all of $\mathbb{C} \cup\{\infty\} \backslash \mathcal{L}$, that is, a covering map, albeit not the universal cover.

A major role will be played by automorphic and character automorphic functions. These are functions, $f$, defined on $\mathbb{D}$ (usually analytic 
but occasionally meromorphic and occasionally only harmonic) or on $\mathbb{C} \cup\{\infty\} \backslash \mathcal{L}$ (always meromorphic) which obey

$$
f(\gamma(z))=c(\gamma) f(z)
$$

for all $\gamma$ and $z$. Here $c \neq 0$ and must obey

$$
c\left(\gamma \gamma^{\prime}\right)=c(\gamma) c\left(\gamma^{\prime}\right)
$$

If $c \equiv 1, f$ is called automorphic. If $|c|=1$ so that $c$ is a unitary group character, we call $f$ character automorphic. A character is determined by $\left\{c\left(\gamma_{j}\right)\right\}_{j=1}^{\ell}$ which can be chosen independently so the set of all characters is an $\ell$-dimensional torus, $\Gamma^{*}$. This set has a group structure if $c \tilde{c}(\gamma)=c(\gamma) \tilde{c}(\gamma)$ is the product and $c(\gamma)=1$ the identity. Moreover, $c^{-1}(\gamma)=\overline{c(\gamma)}$

Notice that $\mathbf{x}$ is automorphic on $\mathbb{D}$ and $\mathbf{x}^{\sharp}$ is automorphic on $\mathbb{C} \cup\{\infty\} \backslash \mathcal{L}$ if we extend the notion to include $\mathcal{S}$-valued functions. Moreover, $f$ is automorphic and analytic (resp. meromorphic) on $\mathbb{D}$ if and only if there is a function, $F$, on $\mathcal{S}_{+}$which is analytic (resp. meromorphic) with

$$
F(\mathbf{x}(z))=f(z)
$$

Similarly, 2.27) with $\mathbf{x}$ replaced by $\mathbf{x}^{\sharp}$ sets up a one-one correspondence between meromorphic functions on $\mathcal{S}$ and meromorphic automorphic functions on $\mathbb{C} \cup\{\infty\} \backslash \mathcal{L}$.

In particular, we have that the analog of 1.19 ,

$$
M(z)=-m(\mathbf{x}(z))
$$

is an automorphic function with $\operatorname{Im} M(z)>0$ for $z \in \mathcal{F}^{\text {int }} \cap \mathbb{C}_{+}$.

We will require the following result:

Theorem 2.1. Fix $\ell$ and let $Q_{\ell} \subset \mathbb{R}^{2 \ell+2}$ be the set of $\left(\alpha_{1}, \beta_{1}, \ldots, \alpha_{\ell+1}, \beta_{\ell+1}\right)$ for which (1.3) holds. Given $q \in Q_{\ell}$, let $\mathbf{x}_{q}$ be the covering map and $\gamma_{j ; q}$ the Fuchsian group generators. Then $\mathbf{x}_{q}$ and $\gamma_{j ; q}$ are continuous in $q$ on $Q_{\ell}$.

This is a special case of a theorem of Hejhal [33] who noted that one can also base a proof using ideas from Ahlfors-Bers [2]. We have found a proof for the case at hand and given it in [65, Sect. 9.8]. We note that the Blaschke product, $B(z)$, that we discuss in Section 4 below is also continuous in $q$.

We will also need the following well-known fact about functions on $\mathcal{S}$ (see [23, 30] or [65, Thm. 5.12.5]):

Theorem 2.2. Let $F$ be a nonconstant meromorphic function on $\mathcal{S}$. Then $F$ has a degree, $d$, so that for all $a,\{w \mid F(w)=a\}$ has $d$ points, counting multiplicity (i.e., $d$ is the sum of the orders of zeros of 
$F(w)-a$ in local coordinates). If $F \circ \tau \not \equiv F$, then the degree of $F$ is at least $\ell+1$.

Remark. In particular, if $F$ is analytic on $\mathcal{S}$ it must be constant.

\section{BeARDON's TheOREM}

From our point of view, a theorem of Beardon [8] plays a critical role. To state the theorem, we need some notions. Fix $s>0$ and a Fuchsian group, $\Gamma$. The Poincaré series is given by

$$
\sum_{\gamma \in \Gamma}\left|\gamma^{\prime}(0)\right|^{s}
$$

We are interested in when this series is convergent. It is a basic fact (see [37, 65]) that if the series in (3.1) converges, then uniformly for $z$ in compacts of $\mathbb{D}$, the series

$$
\sum_{\gamma \in \Gamma}\left|\gamma^{\prime}(z)\right|^{s}
$$

converges, as does uniformly on compacts of $\mathbb{D}$, the series

$$
\sum_{\gamma \in \Gamma}(1-|\gamma(z)|)^{s}
$$

Indeed, convergence of 3.2 for one $z$ implies convergence uniformly on compacts. What is also true (see, e.g., [65, Sect. 9.4]) is that if $K \subset \overline{\mathbb{D}} \backslash \mathcal{L}$ is compact, then there is $C>0$ so that for all $z \in K$ and all $\gamma \in \Gamma$,

$$
\left|\gamma^{\prime}(z)\right| \leq C\left|\gamma^{\prime}(0)\right|
$$

so convergence of (3.1) implies convergence of (3.2) uniformly for $z \in$ $K$. In particular, since $\overline{\mathbb{D}} \backslash \mathcal{L}$ is connected, we see that the series

$$
\sum_{\gamma \in \Gamma}|\gamma(z)-\gamma(w)|^{s}
$$

converges uniformly for $z, w$ in compacts $K \subset \overline{\mathbb{D}} \backslash \mathcal{L}$.

Poincaré [52] proved that for any Fuchsian group, (3.1) converges if $s=2$ and Burnside [12, 13] proved that if the set of limit points is not all of $\partial \mathbb{D}$, then $(3.1)$ converges if $s=1$. Beardon proved

Theorem 3.1 (Beardon [8]). If $\Gamma$ is a finitely generated Fuchsian group whose limit points are not all of $\partial \mathbb{D}$, then there is some $s<1$ so that (3.1) converges. 
As Beardon noted, this is equivalent to the set of limit points having Hausdorff dimension less than 1. Indeed, it is known (work later than Beardon, see [48, 69]) that the infimum over $s$ for which (3.1) converges is the Hausdorff dimension of $\mathcal{L}$. We note that Beardon's proof is very involved, in part because of the need to consider issues such as elliptic and parabolic elements that are irrelevant to our setup. The result for our case is proven using some simple geometry in Simon [65].

There is an important consequence of Beardon's theorem that we need. Let

$$
\tilde{\mathcal{R}}=\partial \mathbb{D} \backslash \partial \mathcal{R}_{0}
$$

that is, $\overline{\mathcal{F}} \cap \partial \mathbb{D}$. This set consists of $2 \ell$ arcs. For each $\gamma \in \Gamma, \gamma(\tilde{\mathcal{R}})$ is also $2 \ell$ arcs, so

$$
\partial \mathcal{R}_{k}=\mathcal{L} \cup\left[\bigcup_{w(\gamma)>k} \gamma(\tilde{\mathcal{R}})\right]
$$

It is not hard to see that on $\tilde{\mathcal{R}}$ and its images, each $\gamma_{j}^{ \pm}$, but one, decreases sizes by a fixed amount so that $(|\cdot|$ is total arc length)

$$
|\gamma(\tilde{\mathcal{R}})| \leq C e^{-D w(\gamma)}
$$

for some fixed constants $C, D>0$ (proven in [65, Sect. 9.6]).

By (3.4), for some constant $Q$,

$$
|\gamma(\tilde{\mathcal{R}})| \leq Q\left|\gamma^{\prime}(0)\right|
$$

Hence,

$$
|\gamma(\tilde{\mathcal{R}})| \leq\left|C e^{-D w(\gamma)}\right|^{1-s}|Q| \gamma^{\prime}(0)||^{s}
$$

So, by Beardon's theorem for some $s<1$,

$$
\left|\partial \mathcal{R}_{k}\right| \leq C^{1-s} Q^{s} e^{-D(1-s) k} \sum_{\gamma \in \Gamma}\left|\gamma^{\prime}(0)\right|^{s}
$$

and thus,

Theorem 3.2. For some constants $C_{0}, D_{0}>0$, we have

$$
\left|\partial \mathcal{R}_{k}\right| \leq C_{0} e^{-D_{0} k}
$$

\section{Blaschke Products and (Potential Theorist's) Green's FUNCTION}

The initial elements of this section are classical; see, for example, Tsuji [73]. Given $w \in \mathbb{D}$, we define $b(z, w)$ by

$$
b(z, w)=\left\{\begin{array}{cc}
\frac{|w|}{w} \frac{w-z}{1-\bar{w} z} & w \neq 0 \\
z & w=0
\end{array}\right.
$$


which is meromorphic in $z$ on $\mathbb{C} \cup\{\infty\}$, analytic in $z$ on $\mathbb{D}$, and is the unique bijective map of $\mathbb{D} \rightarrow \mathbb{D}$ with

$$
b(w, w)=0
$$

and

$$
b(0, w)>0 \quad(w \neq 0) ; \quad b^{\prime}(0, w)>0 \quad(w=0)
$$

Note that $b$ is continuous in $z$ on $\overline{\mathbb{D}}$ and

$$
\left|b\left(e^{i \theta}, w\right)\right|=1
$$

The following is standard (see Rudin [56]):

Lemma 4.1. Let $\left(w_{j}\right)_{j=1}^{\infty}$ be a sequence of points in $\mathbb{D}$. Then

(a) If

$$
\sum_{j=1}^{\infty}\left(1-\left|w_{j}\right|\right)=\infty
$$

then as $N \rightarrow \infty$,

$$
\prod_{j=1}^{N} b\left(z, w_{j}\right) \rightarrow 0
$$

uniformly on compact subsets of $\mathbb{D}$.

(b) If

$$
\sum_{j=1}^{\infty}\left(1-\left|w_{j}\right|\right)<\infty
$$

then as $N \rightarrow \infty$,

$$
\prod_{j=1}^{N} b\left(z, w_{j}\right) \rightarrow B\left(z,\left(w_{j}\right)\right)
$$

uniformly on compact subsets of $\mathbb{D}$, where $B$ is analytic in $\mathbb{D}$ and obeys

$$
B\left(z,\left(w_{j}\right)\right)=0 \Leftrightarrow z \in\left(w_{j}\right)
$$

Moreover, for Lebesgue a.e. $\theta$,

$$
\lim _{r \uparrow 1} B\left(r e^{i \theta},\left(w_{j}\right)\right) \equiv B\left(e^{i \theta},\left(w_{j}\right)\right)
$$

exists obeying

$$
\left|B\left(e^{i \theta},\left(w_{j}\right)\right)\right|=1
$$


Remarks. 1. The refined form of (4.9) says that the order of the zero at some $w_{j}$ is the number of times it occurs in $\left(w_{j}\right)$.

2. The proof shows that when (4.7) holds, uniformly for $|z| \leq \rho<1$, we have

$$
\sum_{j=1}^{\infty}\left|1-b\left(z, w_{j}\right)\right|<\infty
$$

3. The proof of 4.12 follows from the simple inequality

$$
|1-b(z, w)| \leq \frac{1+|z|}{|1-\bar{w} z|}(1-|w|)
$$

which also proves that the product converges on $\mathbb{C} \backslash\left[\overline{\mathbb{D}} \cup\left(1 / \bar{w}_{j}\right)\right]$ and on any set $K \subset \partial \mathbb{D}$ with

$$
\inf _{e^{i \theta} \in K, w_{j}}\left|e^{i \theta}-w_{j}\right|>0
$$

In particular, if there is any set $K \subset \partial \mathbb{D}$ for which (4.14) holds, we can find such an open set and so get a product analytic across $K$. This product is meromorphic, with poles at the points $1 / \bar{w}_{j}$.

We also need the following:

Lemma 4.2. Suppose $\gamma$ is an analytic bijection of $\mathbb{D}$ to $\mathbb{D}$. For any $z, w \in \mathbb{D}$, we have

$$
\begin{array}{ll}
\text { (i) } & |b(z, w)|=|b(w, z)| \\
\text { (ii) } & |b(\gamma(z), \gamma(w))|=|b(z, w)|
\end{array}
$$

Proof. (i) is immediate. For 4.16), fix $w$ and let

$$
h(z)=\frac{b(\gamma(z), \gamma(w))}{b(z, w)}
$$

It is easy to see that $h$ has a removable singularity at $z=w$ and so, it is analytic in $\mathbb{D}$ and continuous on $\overline{\mathbb{D}}$. By $(4.4),\left|h\left(e^{i \theta}\right)\right|=1$ so, by the maximum principle, $|h(z)| \leq 1$ on $\mathbb{D}$. But $1 / h$ has the same properties as $h$, so $|1 / h(z)| \leq 1$, which implies that $|h(z)|=1$, that is, 4.16 holds.

The following is true for any Fuchsian group whose limit points are not dense in $\partial \mathbb{D}$ - but we only care here about the $\Gamma$ 's associated to finite gap sets:

Theorem 4.3. Let $\Gamma$ be the Fuchsian group of a finite gap set. For any $w \in \mathbb{D}$, the product

$$
\prod_{\gamma \in \Gamma} b(z, \gamma(w)) \equiv B(z, w)
$$


converges for all $z \in \mathbb{C} \cup\{\infty\} \backslash\left[\mathcal{L} \cup\left\{\overline{\gamma(w)}^{-1}\right\}_{\gamma \in \Gamma}\right]$ and defines a function analytic there and meromorphic in $\mathbb{C} \cup\{\infty\} \backslash \mathcal{L}$. B has simple poles at the points $\left\{\overline{\gamma(w)}^{-1}\right\}_{\gamma \in \Gamma}$, simple zeros at $\{\gamma(w)\}_{\gamma \in \Gamma}$ and no other zeros or poles. Moreover,

(i) For $z, w \in \mathbb{D}$,

$$
|B(z, w)|=|B(w, z)|
$$

(ii) Each $B(\cdot, w)$ is character automorphic, that is, for every $w \in \mathbb{D}$ there is a character, $c_{w}$, on $\Gamma$ so that

$$
B(\gamma(z), w)=c_{w}(\gamma) B(z, w)
$$

(iii) If

$$
\begin{gathered}
B(z) \equiv B(z, 0) \\
\text { then for } z \in \mathbb{C} \cup\{\infty\} \backslash\left[\mathcal{L} \cup\left\{\overline{\gamma(0)}^{-1}\right\}_{\gamma \in \Gamma}\right], \\
|B(z)|=\prod_{\gamma \in \Gamma}|\gamma(z)|
\end{gathered}
$$

(iv) For $e^{i \theta} \in \partial \mathbb{D} \backslash \mathcal{L}\left(\right.$ with $\left.^{\prime}=\partial / \partial \theta\right)$,

$$
\left|B^{\prime}\left(e^{i \theta}\right)\right|=\sum_{\gamma \in \Gamma}\left|\gamma^{\prime}\left(e^{i \theta}\right)\right|
$$

Remarks. 1. We will see below (Theorem 4.4) that $c_{0}(\gamma)$ is not the identity.

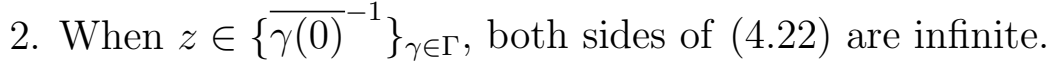

Proof. By Theorem 3.1 (or Burnside's theorem), for any $w \in \mathbb{D}$,

$$
\sum_{\gamma \in \Gamma}|1-\gamma(w)|<\infty
$$

So, by Lemma 4.1 and 4.13), the product converges where claimed and has the claimed analytic/zero/pole properties.

(i) is immediate from 4.15). By the proof of (4.16), there exists $\eta(w, \gamma) \in \partial \mathbb{D}$ so that

$$
b(\gamma(z), \gamma(w))=\eta(w, \gamma) b(z, w)
$$

Thus, for any finite subset, $G \subset \Gamma$,

$$
\begin{aligned}
\prod_{\gamma^{\prime} \in G} b\left(\gamma(z), \gamma^{\prime}(w)\right) & =\prod_{\gamma^{\prime} \in G} b\left(\gamma(z), \gamma\left(\gamma^{-1} \gamma^{\prime}(w)\right)\right) \\
& =\prod_{\gamma^{\prime} \in G} \eta\left(\gamma^{-1} \gamma^{\prime}(w), \gamma\right) \prod_{\gamma^{\prime \prime} \in \gamma^{-1}(G)} b\left(z, \gamma^{\prime \prime}(w)\right)
\end{aligned}
$$


If $G_{n} \subset G_{n+1}$ with $\cup_{n} G_{n}=\Gamma$ and $\gamma$ is fixed, $\gamma^{-1}\left(G_{n}\right) \subset \gamma^{-1}\left(G_{n+1}\right)$ and $\cup_{n} \gamma^{-1}\left(G_{n}\right)=\Gamma$, so the left side of (4.26) and the last factor on the right converge to $B(\gamma(z), w)$ and $B(z, w)$, respectively. Thus, the product of $\eta$ 's converges to some $c_{w}(\gamma) \in \partial \mathbb{D}$, that is, 4.20 holds. From 4.20, it is easy to see that $c_{w}\left(\gamma \gamma^{\prime}\right)=c_{w}(\gamma) c_{w}\left(\gamma^{\prime}\right)$. That proves (ii).

To get 4.22 , suppose first $z \in \mathbb{D}$. Then by 4.19 ,

$$
|B(z)|=|B(z, 0)|=|B(0, z)|=\left|\prod_{\gamma \in \Gamma} \gamma(z)\right|
$$

proving 4.22 for $z \in \mathbb{D}$.

Since for $z \in \mathbb{D}$,

$$
\overline{B(1 / \bar{z})}=B(z)^{-1} \quad \overline{\gamma(1 / \bar{z})}=\gamma(z)^{-1}
$$

(on account of $\left|B\left(e^{i \theta}\right)\right|=\left|\gamma\left(e^{i \theta}\right)\right|=1$ and the reflection principle), we get 4.22 on $\mathbb{C} \cup\{\infty\} \backslash\left[\overline{\mathbb{D}} \cup\left\{\overline{\gamma(0)}^{-1}\right\}_{\gamma \in \Gamma}\right]$. Finally, on $\partial \mathbb{D} \backslash \mathcal{L}$, both sides of (4.22) are 1. This completes (iii).

To prove (iv), we note that if $g$ is an analytic function on $\mathbb{D}$ with $|g(z)|<1$ on $\mathbb{D}$ and $I \subset \partial \mathbb{D}$ is an open interval so that $g$ has an analytic continuation across $I$ with $\left|g\left(e^{i \theta}\right)\right|=1$ on $I$, then

$$
\frac{\partial}{\partial \theta}\left|g\left(e^{i \theta}\right)\right|=0
$$

so, by Cauchy-Riemann equations,

$$
\left.\frac{\partial}{\partial r} \arg \left(g\left(r e^{i \theta}\right)\right)\right|_{r=1}=0
$$

and

$$
\frac{\partial}{\partial \theta} \arg \left(g\left(e^{i \theta}\right)\right)=\left.\frac{\partial}{\partial r}\left|g\left(r e^{i \theta}\right)\right|\right|_{r=1}>0
$$

Hence, with ${ }^{\prime}=\frac{\partial}{\partial \theta}$,

$$
\left|g^{\prime}\left(e^{i \theta}\right)\right|=\left.\frac{\partial}{\partial r}\left|g\left(r e^{i \theta}\right)\right|\right|_{r=1}=\left|\frac{d g}{d z}\left(e^{i \theta}\right)\right|
$$

and

$$
g^{\prime}\left(e^{i \theta}\right)=\left|g^{\prime}\left(e^{i \theta}\right)\right| g\left(e^{i \theta}\right)
$$

In 4.30, we have strict positivity by the same argument that shows boundary values of Herglotz functions are strictly monotone.

Let $G$ be a finite subset of $\Gamma$ and

$$
B_{G}(z)=\prod_{\gamma \in G} b(z, \gamma(0))
$$


Then

$$
\left.\frac{\partial}{\partial r}\left|B_{G}(z)\right|\right|_{z=e^{i \theta}}=\left.\sum_{\gamma \in G} \frac{\partial}{\partial r}|b(z, \gamma(0))|\right|_{z=e^{i \theta}}
$$

by Leibnitz's rule and $\left|b\left(e^{i \theta}, \gamma(0)\right)\right|=1$. If $z_{0} \in \partial \mathbb{D} \backslash \mathcal{L}, B_{G}(z) \rightarrow$ $B(z)$ for $z$ in a neighborhood of $z_{0}$, so derivatives converge. Since $\frac{\partial}{\partial r}\left|B_{G}(z)\right|=\left|\frac{d}{d z} B_{G}(z)\right|$, the $\frac{\partial}{\partial r}$ derivatives converge. The terms in the sum are positive, so the sum over all of $\Gamma$ is absolutely convergent and (4.34) extends to the limit. By 4.30-4.31), we get 4.23).

We emphasize for later use that (4.30-4.31) imply

$$
\frac{\partial}{\partial \theta} \arg \left(\gamma\left(e^{i \theta}\right)\right)=\left|\gamma^{\prime}\left(e^{i \theta}\right)\right|>0
$$

Recall that the (real-valued) potential theoretic Green's function, $G_{\mathfrak{e}}$, is uniquely determined by requiring $G_{\mathfrak{e}}(z)-\log |z|$ to be harmonic on $\mathbb{C} \cup\{\infty\} \backslash \mathfrak{e}$, and for quasi-every $x \in \mathfrak{e}$,

$$
\lim _{\substack{z \rightarrow x \\ z \notin \mathfrak{e}}} G_{\mathfrak{e}}(z)=0 .
$$

In fact, when $\mathfrak{e}$ has the form $11.1-(1.3), G_{\mathfrak{e}}$ can be chosen globally continuous on $\mathbb{C}$ with

$$
G_{\mathfrak{e}} \uparrow \mathfrak{e}=0
$$

Moreover, near infinity,

$$
G_{\mathfrak{e}}(z)=\log |z|-\log (\operatorname{cap}(\mathfrak{e}))+O\left(z^{-1}\right)
$$

and, by the reflection principle, $G_{\mathfrak{e}}(z)$ is real analytic in $x$ and $|y|$ near any $z_{0} \in \mathfrak{e}^{\text {int }}$. For $x \in \mathfrak{e}^{\text {int }}$, we have

$$
\rho_{\mathfrak{e}}(x)=\lim _{\varepsilon \downarrow 0} \frac{1}{\pi} \frac{\partial G_{\mathfrak{e}}}{\partial y}(x+i \varepsilon)
$$

which we will write as

$$
\frac{1}{\pi} \frac{\partial}{\partial n} G_{\mathfrak{e}}(x+i 0)
$$

the normal derivative in the positive direction.

Theorem 4.4. Let $\Gamma$ be the Fuchsian group of a finite gap set, $\mathfrak{e}$. Then for all $z \in \mathbb{D} \backslash\{0\}$,

$$
|B(z)|=e^{-G_{\mathfrak{e}}(\mathbf{x}(z))}
$$

Moreover,

(i) If $x_{\infty}$ is given by

$$
\mathbf{x}(z)=\frac{x_{\infty}}{z}+O(1)
$$


near $z=0$, then, also near $z=0$,

$$
B(z)=\frac{\operatorname{cap}(\mathfrak{e})}{x_{\infty}} z+O\left(z^{2}\right)
$$

(ii) The character, $c_{0}$, of $B(z)$ is given by

$$
c_{0}\left(\gamma_{j}\right)=\exp \left(2 \pi i \rho_{\mathfrak{e}}\left(\left[\alpha_{1}, \beta_{j}\right]\right)\right)
$$

(iii) At any $x_{0} \in\left\{\alpha_{j}, \beta_{j}\right\}_{j=1}^{\ell+1}, G_{\mathfrak{e}}$ has a square root zero in the sense that

$$
\begin{aligned}
& \lim _{x \uparrow \alpha_{j}} G_{\mathfrak{e}}(x)\left(\alpha_{j}-x\right)^{-1 / 2}=a_{j} \\
& \lim _{x \downarrow \beta_{j}} G_{\mathfrak{e}}(x)\left(x-\beta_{j}\right)^{-1 / 2}=b_{j}
\end{aligned}
$$

for nonzero $a_{j}, b_{j}$.

Proof. By 4.20), $|B(z)|$ is automorphic, so there exists a function $h$ on $\mathbb{C} \cup\{\infty\} \backslash \mathfrak{e}$ such that

$$
|B(z)|=h(\mathbf{x}(z))
$$

Since $\mathbf{x}$ is analytic and $\mathbf{x} B$ is nonvanishing and analytic in a neighborhood of $\overline{\mathcal{F}}, \log (h(x))+\log |x|$ is harmonic on $\mathbb{C} \cup\{\infty\} \backslash \mathfrak{e}$. By $|B(z)|=1$ on $\overline{\mathcal{F}} \cap \partial \mathbb{D}, \log (h(x)) \rightarrow 0$ as $x \rightarrow \mathfrak{e}$. Thus, $-\log (h(x))$ is $G_{\mathfrak{e}}(x)$, proving 4.41 ).

(4.43) is immediate from $(4.42)$ and 4.38$)$. 4.45) and 4.46) follow from $(4.41), B^{\prime}(z) \neq 0$ on $\overline{\mathcal{F}} \cap \partial \mathbb{D}$, and the fact that on $\mathbf{x}^{-1}\left(\left\{\alpha_{j}, \beta_{j}\right\}_{j=1}^{\ell+1}\right)$, we have $\mathbf{x}^{\prime}(z)=0, \mathbf{x}^{\prime \prime}(z) \neq 0$. That leaves (4.44).

Consider the generator, $\gamma_{\ell}$, whose action takes 0 into the endpoint of the curve in the top of Figure 2. Since

$$
B\left(\gamma_{\ell}(0)\right)=c_{0}\left(\gamma_{\ell}\right) B(0)
$$

we see that

$$
\arg \left(c_{0}\left(\gamma_{\ell}\right)\right)=\oint_{0}^{\gamma_{\ell}(0)} \frac{d}{d z} \arg (B(z)) d z
$$

$G_{\mathfrak{e}}(z)$ is harmonic on $\mathbb{C} \backslash \mathfrak{e}$ so that, locally, it is the real part of an analytic function, $\tilde{G}_{\mathfrak{e}}(z)$, but that function has a multivalued imaginary part. Thus, $e^{-\tilde{G}_{\mathfrak{e}}(z)} \equiv E(z)$ has a multivalued argument. Clearly,

$$
B(z)=E(\mathbf{x}(z))
$$

and the change of argument in 4.49 is given by the change of argument of $E(z)$ over the curve in the bottom of Figure 2. This is given by a sum of change of argument around curves surrounding each band, since in the gaps and in $\left(-\infty, \alpha_{1}\right)$, there is cancellation between top and bottom. 
By a Cauchy-Riemann equation,

$$
\frac{\partial}{\partial x} \arg (E(z))=\frac{\partial}{\partial y} \log |E(z)|=\frac{\partial G_{\mathrm{e}}}{\partial n}
$$

The normal derivatives on top and bottom of a band have opposite sign, so given the opposite directions,

$$
\begin{aligned}
\arg \left(c_{0}\left(\gamma_{\ell}\right)\right) & =\sum_{j=1}^{\ell} \int_{\alpha_{j}}^{\beta_{j}} 2 \frac{\partial G_{\mathfrak{e}}(x+i 0)}{\partial n} d x \\
& =2 \pi \sum_{j=1}^{\ell} \int_{\alpha_{j}}^{\beta_{j}} \rho_{\mathfrak{e}}(x) d x=2 \pi \rho_{\mathfrak{e}}\left(\left[\alpha_{1}, \beta_{\ell}\right]\right)
\end{aligned}
$$

which is 4.44 for $j=\ell$. 4.52 follows from 4.39 . The argument for general $j=1, \ldots, \ell-1$ is similar.

Corollary 4.5. $B(z)^{p}$ is automorphic if and only if

$$
\rho_{\mathfrak{e}}\left(\left[\alpha_{j}, \beta_{j}\right]\right)=\frac{q_{j}}{p}
$$

for integers $q_{j}$.

Remark. We will eventually see (Corollary 6.4) that this relates periodic $\mathfrak{e}$ to periodic Jacobi matrices.

Proof. $B(z)^{p}$ is automorphic if and only if $c_{0}\left(\gamma_{j}\right)^{p}=1$ for $j=1,2, \ldots, \ell$ and this, given (4.44), is equivalent to 4.53).

Corollary 4.6. Let $\mathfrak{e}$ be a finite gap set and $d \rho_{\mathfrak{e}}$ its equilibrium measure. Then

$$
\int_{\partial \mathbb{D}} f\left(\mathbf{x}\left(e^{i \theta}\right)\right) \frac{d \theta}{2 \pi}=\int_{\mathfrak{e}} f(x) d \rho_{\mathfrak{e}}(x)
$$

for all continuous $f$ on $\mathfrak{e}$ and, if integrals are allowed to be infinite, for any positive measurable $f$ on $\mathfrak{e}$. In particular, $f \in L^{p}\left(\mathfrak{e}, d \rho_{\mathfrak{e}}\right)$ if and only if $f \circ \mathbf{x} \in L^{p}\left(\partial \mathbb{D}, \frac{d \theta}{2 \pi}\right)$ so the Szego" conditions,

$$
\int_{\partial \mathbb{D}} \log \left(f\left(\mathbf{x}\left(e^{i \theta}\right)\right)\right) \frac{d \theta}{2 \pi}>-\infty
$$

and

$$
\int_{\mathfrak{e}} \log (f(x)) d \rho_{\mathfrak{e}}(x)>-\infty
$$

are equivalent for $f \in L^{1}\left(\mathfrak{e}, d \rho_{\mathfrak{e}}\right)$. 
Proof. It suffices to prove 4.54 for continuous functions, $f$, and then use standard approximation arguments. Recall that $\tilde{\mathcal{R}}$ is given by (3.6) and consists of $2 \ell$ arcs. Except for endpoints, it is a fundamental domain for the action of $\Gamma$ on $\partial \mathbb{D}$, so

$$
\begin{aligned}
\int_{\partial \mathbb{D}} f\left(\mathbf{x}\left(e^{i \theta}\right)\right) \frac{d \theta}{2 \pi} & =\sum_{\gamma \in \Gamma} \int_{\gamma(\tilde{\mathcal{R}})} f\left(\mathbf{x}\left(e^{i \theta}\right)\right) \frac{d \theta}{2 \pi} \\
& =\sum_{\gamma \in \Gamma} \int_{\tilde{\mathcal{R}}} f\left(\mathbf{x}\left(e^{i \theta}\right)\right)\left|\gamma^{\prime}\left(e^{i \theta}\right)\right| \frac{d \theta}{2 \pi}
\end{aligned}
$$

by a change of variables and the invariance of $\mathbf{x}$ under $\Gamma$, that is, $\mathbf{x} \circ \gamma=\mathbf{x}$. Thus, by 4.23,

$$
\begin{aligned}
\int_{\partial \mathbb{D}} f\left(\mathbf{x}\left(e^{i \theta}\right)\right) \frac{d \theta}{2 \pi} & =\int_{\tilde{\mathcal{R}}} f\left(\mathbf{x}\left(e^{i \theta}\right)\right)\left|B^{\prime}\left(e^{i \theta}\right)\right| \frac{d \theta}{2 \pi} \\
& =\int_{\tilde{\mathcal{R}}} f\left(\mathbf{x}\left(e^{i \theta}\right)\right)\left|\frac{\partial}{\partial n} e^{-G_{\mathfrak{e}}\left(\mathbf{x}\left(e^{i \theta}\right)\right)}\right| \frac{d \mathbf{x}\left(e^{i \theta}\right)}{d \theta} \frac{d \theta}{2 \pi}
\end{aligned}
$$

where we use 4.41), $\left|B^{\prime}\left(e^{i \theta}\right)\right|=\left.\frac{d}{d r}\left|B\left(r e^{i \theta}\right)\right|\right|_{r=1}$, and the chain rule to go from $d \theta$ to $d \mathbf{x}$ derivatives. $\mathbf{x}$ on $\tilde{\mathcal{R}}$ is a two-fold cover of $\mathfrak{e}$, so using $\frac{\partial}{\partial n} e^{-G_{\mathfrak{e}}(\mathbf{x})}=-\frac{\partial}{\partial n} G_{\mathfrak{e}}\left(\right.$ since $G_{\mathfrak{e}}$ is 0 on $\mathfrak{e}$ ) and $4.39-4.40$, we get

$$
\int_{\partial \mathbb{D}} f\left(\mathbf{x}\left(e^{i \theta}\right)\right) \frac{d \theta}{2 \pi}=2 \int_{\mathfrak{e}} \pi \rho_{\mathfrak{e}}(x) f(x) \frac{d x}{2 \pi}=\int_{\mathfrak{e}} f(x) d \rho_{\mathfrak{e}}(x)
$$

That concludes what we need about Blaschke products in this paper, but we put in some results on products of Blaschke products which will be critical in later papers in this series.

Theorem 4.7. Let $\left(w_{k}\right)_{k=1}^{\infty}$ be a sequence in $\mathcal{F}$. Then

$$
\sum_{k=1}^{\infty}\left(1-\left|w_{k}\right|\right)<\infty \Leftrightarrow \sum_{k=1}^{\infty}\left(1-\left|B\left(w_{k}\right)\right|\right)<\infty
$$

Moreover,

(i) If $\sum_{k=1}^{\infty}\left(1-\left|w_{k}\right|\right)=\infty$, then $\prod_{k=1}^{N} B\left(z, w_{k}\right)$ converges to 0 uniformly on compact subsets of $\mathbb{D}$.

(ii) If

$$
\sum_{k=1}^{\infty}\left(1-\left|w_{k}\right|\right)<\infty
$$


then for all $z \in \mathbb{D}$,

$$
\sum_{k=1}^{\infty}\left|1-B\left(z, w_{k}\right)\right|<\infty
$$

uniformly on compact subsets of $\mathbb{D}$, so $\prod_{k=1}^{N} B\left(z, w_{k}\right)$ converges to an analytic limit vanishing if and only if $z \in\left\{\gamma\left(w_{k}\right)\right\}_{\gamma \in \Gamma, k=1, \ldots}$.

Remarks. 1. If $\left\{\gamma_{k}\right\}$ is a countable set of distinct elements in $\Gamma$ and $w_{k}=\gamma_{k}(0)$, then $\sum_{k=1}^{\infty}\left(1-\left|w_{k}\right|\right)<\infty$ (by Burnside or Beardon), but $\left|B\left(z, \gamma_{k}(0)\right)\right|=|B(z)|$ and $\prod_{k=1}^{N} B\left(z, w_{k}\right)$ converges to 0 uniformly on compact subsets of $\mathbb{D}$. Thus, the condition $w_{k} \in \mathcal{F}$ cannot be replaced by $w_{k} \in \mathbb{D}$ in (ii).

2. As in the case of Theorem 4.3, we can prove convergence on open subsets of $\mathbb{C} \cup\{\infty\} \backslash\left[\mathcal{L} \cup\left\{{\overline{\gamma\left(w_{k}\right)}}^{-1}\right\}_{\gamma \in \Gamma, k=1, \ldots]}\right.$ with poles at $\left\{{\overline{\gamma\left(w_{k}\right)}}^{-1}\right\}_{\gamma \in \Gamma, k=1, \ldots}$.

Proof. $B$ is analytic in a neighborhood of $\overline{\mathcal{F}} \cap \mathbb{D}$, as noted in 4.30), $\left|B^{\prime}\left(e^{i \theta}\right)\right| \neq 0$, and, of course, $\left|B\left(e^{i \theta}\right)\right|=1$. Thus, for nonzero constants, $c, d$, and for all $w \in \mathcal{F}$,

$$
c(1-|B(w)|) \leq 1-|w| \leq d(1-|B(w)|)
$$

from which 4.62 is immediate. (Notice that 4.65 only holds on $\mathcal{F}$, not on $\mathbb{D}$, and is where the condition $w_{k} \in \mathcal{F}$ is used.)

To prove (i), we need only note that

$$
\left|B\left(z, w_{k}\right)\right| \leq\left|b\left(z, w_{k}\right)\right|
$$

and use Lemma 4.1(a).

To prove (ii), we note that $\prod_{k=1}^{\infty} B\left(z, w_{k}\right)$ is a product of Blaschke products, so to prove 4.64 , it suffices to prove that

$$
\sum_{\gamma \in \Gamma, k}\left(1-\left|\gamma\left(w_{k}\right)\right|\right)<\infty
$$

Since $\sum_{\gamma \in \Gamma}(1-|\gamma(0)|)<\infty$ and zero occurs at most finitely often in the sequence $\left(w_{k}\right)_{k=1}^{\infty}$ if 4.63 holds, we can suppose that no $w_{k}$ is zero, in which case, since $w_{k} \in \mathcal{F}$ implies (by 2.13)

$$
\left|w_{k}\right|=\inf _{\gamma \in \Gamma}\left|\gamma\left(w_{k}\right)\right|
$$

we have

$$
\inf _{\gamma \in \Gamma, k}\left|\gamma\left(w_{k}\right)\right|>0
$$


This implies that 4.67) is equivalent to

$$
\prod_{\gamma \in \Gamma, k}\left|\gamma\left(w_{k}\right)\right|>0
$$

which, by 4.22 , is equivalent to

$$
\prod_{k}\left|B\left(w_{k}\right)\right|>0
$$

Now, 4.71 is implied by

$$
\sum_{k}\left(1-\left|B\left(w_{k}\right)\right|\right)<\infty
$$

As we have seen, (4.63) implies 4.72), and thus (4.67).

We are especially interested in the case where $w_{k}$ is determined by $w_{k} \in \mathcal{F}$ and $\mathbf{x}\left(w_{k}\right)$ real (so $w_{k} \in\left[\cup_{j=1}^{\ell} C_{\ell}^{+}\right] \cup(-1,1)$ ). In that case,

Proposition 4.8. Let $\left(x_{k}\right)_{k=1}^{\infty}$ be a sequence in $\mathbb{R} \backslash \mathfrak{e}$ and let $w_{k} \in \mathcal{F}$ be uniquely determined by

$$
\mathbf{x}\left(w_{k}\right)=x_{k}
$$

Then the following are equivalent:

$$
\begin{array}{ll}
\text { (i) } & \sum_{k=1}^{\infty}\left(1-\left|w_{k}\right|\right)<\infty \\
\text { (ii) } & \sum_{k=1}^{\infty} G_{\mathfrak{e}}\left(x_{k}\right)<\infty \\
\text { (iii) } & \sum_{k=1}^{\infty} \operatorname{dist}\left(x_{k}, \mathfrak{e}\right)^{1 / 2}<\infty
\end{array}
$$

Proof. By 4.45- 4.46), we have (ii) $\Leftrightarrow$ (iii). By 4.62 , we have that (i) is equivalent to

$$
\sum_{k=1}^{\infty}\left(1-\left|B\left(w_{k}\right)\right|\right)<\infty
$$

which, by 4.41 and 4.73), is equivalent to

$$
\sum_{k=1}^{\infty}\left|1-e^{-G_{\mathfrak{e}}\left(x_{k}\right)}\right|<\infty
$$

In turn, 4.78 is easily seen to be equivalent to 4.75).

Finally, we need to discuss alternating Blaschke products. We will discuss a case with points approaching the top of a gap (or $\alpha_{1}$ ). A similar result holds for approach to a $\beta_{j}$. 
Theorem 4.9. Suppose $\left(\zeta_{k}\right)_{k=1}^{\infty},\left(\rho_{k}\right)_{k=1}^{\infty}$ obey, for some $j$,

$$
\beta_{j-1}<\zeta_{1}<\rho_{1}<\zeta_{2}<\rho_{2}<\cdots<\alpha_{j}
$$

(where $\left.\beta_{0} \equiv-\infty\right)$ and

$$
\lim _{k \rightarrow \infty} \zeta_{k}=\alpha_{j}
$$

Let $\left\{z_{k}\right\} \cup\left\{p_{k}\right\} \subset \mathcal{F}$ and $a_{j} \in \overline{\mathcal{F}}$ be given by

$$
\mathbf{x}\left(z_{k}\right)=\zeta_{k} \quad \mathbf{x}\left(p_{k}\right)=\rho_{k} \quad \mathbf{x}\left(a_{j}\right)=\alpha_{j}
$$

Then as $N \rightarrow \infty$,

$$
\prod_{k=1}^{N} \frac{B\left(z, z_{k}\right)}{B\left(z, p_{k}\right)} \rightarrow B_{\infty}(z)
$$

uniformly in $z$ on compact subsets of

$$
\mathbb{C} \cup\{\infty\} \backslash\left[\mathcal{L} \cup\left\{\gamma\left(p_{k}\right), \gamma\left(z_{k}^{-1}\right)\right\}_{\gamma \in \Gamma, k=1, \ldots} \cup\left\{\gamma\left(a_{j}\right)\right\}_{\gamma \in \Gamma}\right]
$$

to a function which is analytic on the set in 4.82 with simple poles at points in $\left\{\gamma\left(p_{k}\right), \gamma\left(z_{k}^{-1}\right)\right\}_{\gamma \in \Gamma, k=1, \ldots}$ and with zeros only at the points $\left\{\gamma\left(p_{k}^{-1}\right), \gamma\left(z_{k}\right)\right\}_{\gamma \in \Gamma, k=1, \ldots}$.

Moreover,

$$
z \in \partial \mathbb{D} \backslash\left[\mathcal{L} \cup\left\{\gamma\left(a_{j}\right)\right\}_{\gamma \in \Gamma}\right] \Rightarrow\left|B_{\infty}(z)\right|=1
$$

and for some constant $C$ (e-dependent),

$$
z \in \mathcal{F} \Rightarrow\left|\arg \left(B_{\infty}(z)\right)\right| \leq C
$$

if $\arg \left(B_{\infty}\right)$ is determined by requiring one value of $\arg \left(B_{\infty}(0)\right)$ to be zero.

In addition, if we remove the arc of $C_{j-1}^{+}$(or segment of $[-1,0)$ if $j=1)$ that runs from $z_{1}$ to $a_{j}$ and all its images under $\gamma \in \Gamma$, we get a region, $\mathcal{B}$, free of zeros and poles of $B_{\infty}$, on which

$$
z \in \mathcal{B} \backslash \mathcal{R}_{n+1} \Rightarrow\left|\arg \left(B_{\infty}(z)\right)\right| \leq(2 n+1) C
$$

Remarks. 1. If $z_{k} \in C_{j}^{+}$for some $j$, then $\gamma_{j}^{-1}\left(z_{k}\right)=\bar{z}_{k}$, so $\left\{\gamma\left(z_{k}^{-1}\right)\right\}_{\gamma \in \Gamma}=\left\{\gamma\left(\bar{z}_{k}^{-1}\right)\right\}_{\gamma \in \Gamma}$, which is why we do not need to put complex conjugates in 4.82.

2. The analog of this result for $\mathfrak{e}=[-2,2]$ is from Simon [57].

Sketch (see [65] for details). One first shows that if $\zeta, \omega$ run through a compact set, $Q$, in a single $C_{j}^{+}$(including the endpoints) or $[-1,0$ ) or $(0,1]$ and $z$ through a compact subset, $K$, of $\mathbb{C} \backslash \mathcal{L} \cup\left[\{\gamma(Q)\}_{\gamma \in \Gamma} \cup\right.$ $\left.\left\{\gamma\left(Q^{-1}\right)\right\}_{\gamma \in \Gamma}\right]$, then there is $C<\infty$ so that for all $\gamma \in \Gamma, \zeta, \omega \in Q$ and $z \in K$,

$$
|b(z, \gamma(\zeta))-b(z, \gamma(\omega))| \leq C|\gamma(\zeta)-\gamma(\omega)|
$$


This comes from looking at the three parts of

$$
b(z, w)=\frac{|w|}{w} \frac{w-z}{1-\bar{w} z}
$$

From this and telescoping, one gets

$$
|B(z, \zeta)-B(z, \omega)| \leq C \sum_{\gamma \in \Gamma}|\gamma(\zeta)-\gamma(\omega)| \leq C_{1}|\zeta-\omega|
$$

Since

$$
\inf _{\substack{z \in K \\ w \in Q}}|B(z, w)|>0
$$

4.88) implies that

$$
\left|1-\frac{B(z, \zeta)}{B(z, \omega)}\right| \leq C_{2}|\zeta-\omega|
$$

which leads to the convergence of 4.81 if we note that

$$
\sum_{k=1}^{\infty}\left|z_{k}-p_{k}\right|<\infty
$$

since the sum is bounded by the arclength of $C_{j}^{+}$(or by 1 if $\alpha_{j}=\alpha_{1}$ ). This easily leads to all the statements except those about $\arg \left(B_{\infty}\right)$.

For any smooth function, $f$, on a circle $C=\left\{z \mid z=z_{0}+r e^{i \theta}\right\}$, define

$$
\operatorname{Var}_{C}(f)=\int_{0}^{2 \pi}\left|\frac{d}{d \theta} f(z)\right| d \theta
$$

For $w$ outside $C$, let

$$
f_{w}(z)=\arg (w-z)
$$

Then this arg is increasing on one arc between the tangents to $C$ from $w$ and decreasing on the other, so

$$
\operatorname{Var}_{C}\left(f_{w}\right)=2 \times \text { angle between tangents }
$$

This shows that

$$
\operatorname{Var}_{C}\left(f_{w}\right) \leq 2 \pi
$$

and

$$
\operatorname{Var}_{C}\left(f_{w}\right) \leq \frac{4 \operatorname{radius}(C)}{\operatorname{dist}(w, C)}
$$

Since $\arg (B(z, w))$ is built out of $\operatorname{such} \arg (z-\gamma(w))$ and $\arg (z-$ $\left.\overline{\gamma(w)}^{-1}\right)$, and the radii of the circles containing $\gamma\left(C_{j}^{+}\right)$decrease so fast that, by Beardon's theorem,

$$
\sum_{\gamma \in \Gamma} \operatorname{radius}\left(\gamma\left(C_{j}^{+}\right)\right)<\infty
$$


we find, uniformly for $z \in \mathcal{F}$, that

$$
\operatorname{Var}_{C_{j}^{+}}(\arg (B(z, \cdot))) \leq C_{0}
$$

for some finite constant $C_{0}$.

Consider $\arg \left(B_{\infty}\left(z ;\left\{z_{k}\right\},\left\{p_{k}\right\}\right)\right)$ as $p_{k}$ is changed from $z_{k}$ to its final value. At $p_{k} \equiv z_{k}$, this arg is 0 and the total change is bounded by the variation of $\arg (B(z, w))$ as $w$ varies over $C_{j-1}^{+}$. We conclude that on $\mathcal{F}$

$$
\left|\arg \left(B_{\infty}(z)\right)\right| \leq C_{0}
$$

proving (4.84). Since $B_{\infty}$ is character automorphic, the variation of $\arg \left(B_{\infty}(z)\right)$ over any $\gamma(\mathcal{F})$ is at most $2 C_{0}$, from which 4.85$)$ is immediate.

\section{Theta Functions And Abel's Theorem}

Given a general compact Riemann surface, $\mathcal{S}$, of genus $\ell$, one can construct a natural map, $\mathfrak{A}$, called the Abel map from $\mathcal{S}$ to a $2 \ell$-dimensional real torus, called the Jacobi variety, realized as $\mathbb{C}^{\ell} / \mathbb{L}$ where $\mathbb{L}$ is a $2 \ell$ dimensional lattice. Once a base point in $\mathcal{S}$ is fixed, the group structure comes into play. The theory of meromorphic functions - essentially, which finite subsets of $\mathcal{S}$ can occur as zeros and poles - is described using $\mathfrak{A}$ via a result called Abel's theorem.

As we will see in the next section, certain $m$-functions of Jacobi matrices with $\sigma_{\text {ess }}(J)=\mathfrak{e}$ define meromorphic functions on the Riemann surface, $\mathcal{S}$, constructed in Section 2. Their zeros and poles lie only at $\infty_{+}, \infty_{-}$, or in the sets $G_{j}$ of $(2.7)$. $\mathfrak{A}$ takes $\cup_{j} G_{j}$ into an $\ell$-dimensional torus inside the $2 \ell$-dimensional Jacobi variety (the real part of the Jacobi variety), which is also a subgroup with a suitable choice of base point. The traditional construction of the isospectral torus $([21,24,39,44,78])$ uses this general theory of the Abel map and Abel's theorem.

Here, following Sodin-Yuditskii [66], we restrict ourselves to meromorphic functions with poles and zeros only at $\infty_{+}, \infty_{-}$, and in $\cup_{j} G_{j}$. In that case everything can be made explicit in a way that the real part of the Jacobi variety becomes just the $\ell$-dimensional torus, $\Gamma^{*}$, of characters for the Fuchsian group, $\Gamma$. The key is the definition of some natural functions on $\mathbb{C} \cup\{\infty\} \backslash \mathcal{L}$ parametrized by points in $\cup_{j=1}^{\ell} \tilde{C}_{j}^{+}$ (defined below). Our construction is motivated by the one in [66] but is more explicit. 
We will need to use a fundamental set of the action of $\Gamma$ on $\mathbb{C} \cup$ $\{\infty\} \backslash \mathcal{L}$

$$
\tilde{\mathcal{F}}=\left(\overline{\mathcal{F}} \cup \overline{\mathcal{F}}^{-1}\right) \backslash \bigcup_{j=1}^{\ell} \tilde{C}_{j}^{-}
$$

where closure is taken in $\mathbb{C}$, and $\tilde{C}_{j}^{ \pm}$are the complete orthocircles (obtained by adding the two missing points on $\partial \mathbb{D}$ to $C_{j}^{ \pm} \cup\left(C_{j}^{\mp}\right)^{-1}$, respectively); see Figure 4 below. $\tilde{\mathcal{F}}^{\text {int }}$ will denote its interior, this is a fundamental region. $\tilde{\mathcal{F}}$ is then $\tilde{\mathcal{F}}^{\text {int }}$ with $\cup_{j=1}^{\ell} \tilde{C}_{j}^{+}$added.

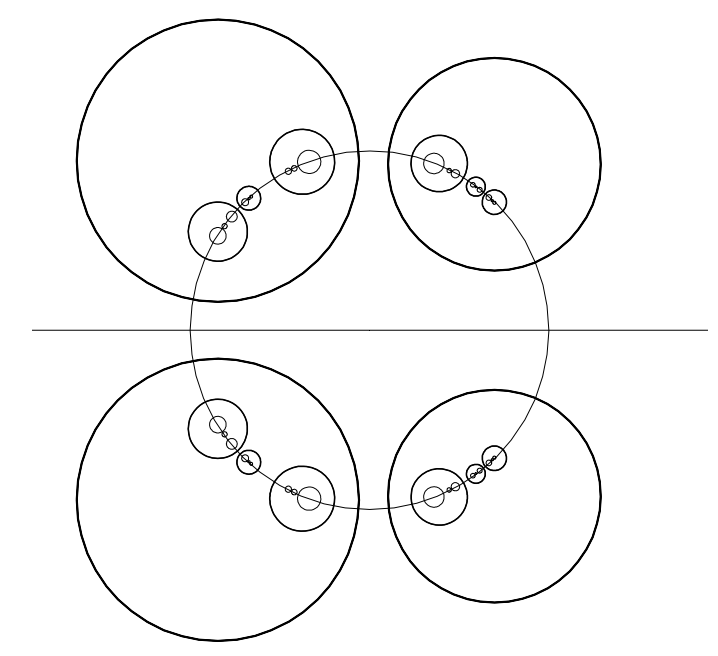

Figure 4. Complete orthocircles

$\mathbf{x}^{\sharp}$ maps $\tilde{C}_{j}^{+}$onto $G_{j}$ bijectively. We will use $\zeta_{1}, \ldots, \zeta_{\ell}$ for the unique points on $\tilde{C}_{1}^{+}, \ldots, \tilde{C}_{\ell}^{+}$that map to $\beta_{1}, \ldots, \beta_{\ell}$, that is,

$$
\zeta_{j} \in \tilde{C}_{j}^{+} \cap \partial \mathbb{D} \quad \mathbf{x}^{\sharp}\left(\zeta_{j}\right)=\beta_{j}
$$

We need the following lemma:

Lemma 5.1. Let $f$ be a character automorphic meromorphic function on $\mathbb{C} \cup\{\infty\} \backslash \mathcal{L}$. Suppose

(i) $f$ has no zeros or poles in $\tilde{\mathcal{F}}^{\text {int }}$ (so in $\tilde{\mathcal{F}}$, the only zeros and poles are on $\left.\cup_{j=1}^{\ell} \tilde{C}_{j}^{+}\right)$.

(ii) Every zero or pole of $f$ has even order. 
(iii) If $D_{j}$ is a counterclockwise contour that is just outside $\tilde{C}_{j}^{+}$(say, a circle with the same center but a slightly larger radius), then

$$
\frac{1}{2 \pi i} \oint_{D_{j}} \frac{f^{\prime}(z)}{f(z)} d z=0
$$

(iv)

$$
f(0)>0
$$

Then there is a (unique) character automorphic function, $g$, which we will denote as $\sqrt{f}$, with

$$
g(0)>0
$$

and so that for all $z \in \mathbb{C} \cup\{\infty\} \backslash \mathcal{L}$,

$$
g(z)^{2}=f(z)
$$

Proof. By (5.3), we can define a single-valued function $h(z)$ on $\tilde{\mathcal{F}}^{\text {int }}$ by

$$
h(z)=\log (f(0))+\oint_{0}^{z} \frac{f^{\prime}(w)}{f(w)} d w
$$

with any contour in $\tilde{\mathcal{F}}^{\text {int }}$ used. Then

$$
g(z)=\exp \left(\frac{1}{2} h(z)\right)
$$

obeys $5.5-5.6)$ and is defined and analytic on $\tilde{\mathcal{F}}^{\text {int }}$.

Since all poles and zeros on $\tilde{C}_{j}^{+}$are of even order, $g(z)$ can be meromorphically continued to a neighborhood, $N$, of the closure of $\tilde{\mathcal{F}}$. Then for each $j, S_{j} \equiv\left\{z \in \tilde{\mathcal{F}}^{\text {int }} \mid \gamma_{j}(z) \in N\right\}$ is open and nonempty, and by decreasing $N$, one can suppose each $S_{j}$ is connected.

If $z \in S_{j}$, we have $g\left(\gamma_{j}(z)\right)^{2}=c_{f}\left(\gamma_{j}\right) g(z)^{2}$. Hence, by continuity and connectedness, there is a single square root, $c_{g}\left(\gamma_{j}\right)$, so that

$$
z \in S_{j} \Rightarrow g\left(\gamma_{j}(z)\right)=c_{g}\left(\gamma_{j}\right) g(z)
$$

We can use this to extend $g$ to $\cup_{j} \gamma_{j}(\tilde{\mathcal{F}})$ and also to $\cup_{j} \gamma_{j}^{-1}(\tilde{\mathcal{F}})$, and so (5.9) holds for all $z$ with $z$ and $\gamma_{j}(z)$ in the domain of current definition. In this way, one gets a character automorphic continuation of $g$ to $\mathbb{C} \cup\{\infty\} \backslash \mathcal{L}$.

Lemma 5.2. Let $\zeta \in \tilde{C}_{j}^{+}$for some $j$. Then

$$
f(z)=\frac{\mathbf{x}(z)-\mathbf{x}(\zeta)}{\mathbf{x}(z)-\mathbf{x}\left(\zeta_{j}\right)} \eta(z) \eta(0)^{-1}
$$

where

$$
\eta(z)=\left\{\begin{array}{cl}
B(z, \zeta) & \text { if } \zeta \in \mathbb{D} \\
1 & \text { if } \zeta \in \partial \mathbb{D} \\
B\left(z, \bar{\zeta}^{-1}\right)^{-1} & \text { if } \zeta \in \mathbb{C} \backslash \overline{\mathbb{D}}
\end{array}\right.
$$


obeys properties (i)-(iv) of Lemma 5.1. If $\zeta \neq \zeta_{j}$, $f$ has double zeros at $\{\gamma(\zeta)\}_{\gamma \in \Gamma}$, double poles at $\left\{\gamma\left(\zeta_{j}\right)\right\}_{\gamma \in \Gamma}$, and is otherwise finite and nonvanishing.

Remarks. 1. If $\zeta=\zeta_{j}, f \equiv 1$.

2. When $z \in\{\gamma(0)\}_{\gamma \in \Gamma}, \mathbf{x}(z)=\infty$ and the first factor in (5.10) is interpreted as 1 . Thus, because of $\eta(0)^{-1}$, we have $f(0)=1$.

Proof. (i) and (iv) are obvious. Moreover, if $\zeta \neq \zeta_{j}$ then $f(z) / \eta(z)$ has double poles at $\left\{\gamma\left(\zeta_{j}\right)\right\}_{\gamma \in \Gamma}$ (since $\mathbf{x}^{\prime}(z)=0, \mathbf{x}^{\prime \prime}(z) \neq 0$ at such points), double zeros at $\{\gamma(\zeta)\}_{\gamma \in \Gamma}$ if $\zeta$ is the other point on $\tilde{C}_{j}^{+}$in $\partial \mathbb{D}$ (i.e., if $\mathbf{x}^{\sharp}(\zeta)=\alpha_{j}$ ), and simple zeros at $\{\gamma(\zeta)\}_{\gamma \in \Gamma} \cup\left\{\gamma\left(\bar{\zeta}^{-1}\right)\right\}_{\gamma \in \Gamma}$ if $\zeta \notin \partial \mathbb{D}$ ( since $\mathbf{x}^{\prime}(z) \neq 0$ at such points and $\mathbf{x}(\zeta)$ is real). Thus, there are precisely the claimed zeros/poles for $f$ since $\eta$ cancels the zeros at $\left\{\gamma\left(\bar{\zeta}^{-1}\right)\right\}_{\gamma \in \Gamma}$ and doubles the zeros at $\{\gamma(\zeta)\}_{\gamma \in \Gamma}$. This proves (ii).

To prove (iii), we need only check (5.3) if $f$ is replaced by $\eta$ or by $f / \eta . f / \eta$ is real on $\partial \mathbb{D}$, so if $q$ is the composition of this function and a conformal map of $\mathbb{C}$ taking $\mathbb{R}$ to $\partial \mathbb{D}, q$ is real on the set of points in its domain which lie on $\mathbb{R}$. So

$$
\frac{1}{2 \pi i} \oint_{\tilde{D}} q(z) d z=0
$$

for any conjugate symmetric curve, and so by contour deformation, for $D_{j}$ and $(f / \eta)^{\prime} /(f / \eta)$.

For $\eta$, we note that if $f$ in $(5.3)$ is replaced by a finite product $\prod_{\gamma \in G} b(z, \gamma(\zeta))$, the integral is zero since the finite product is meromorphic inside $D_{j}$ with an equal number of (simple) zeros and (simple) poles. By taking limits, (5.3) holds for $B(z, \zeta)$, and by $(1 / g)^{\prime} /(1 / g)=-g^{\prime} / g$ for $B\left(z, \bar{\zeta}^{-1}\right)^{-1}$. Thus, (5.3) holds for $f$.

Definition. Let $y \in G_{j}$ for some $j$ and let $\zeta$ be the unique point in $\tilde{C}_{j}^{+}$with

$$
\mathbf{x}^{\sharp}(\zeta)=y
$$

We define $\Theta(\cdot ; y)$ to be the character automorphic function

$$
\Theta(z ; y)=\left[\left(\frac{\mathbf{x}(z)-\mathbf{x}(\zeta)}{\mathbf{x}(z)-\mathbf{x}\left(\zeta_{j}\right)}\right) \eta(z) \eta(0)^{-1}\right]^{1 / 2}
$$

and denote by $\mathfrak{A}(y) \in \Gamma^{*}$ its character. Moreover, we define $\mathfrak{A}(\infty)$ to be the character of $B(z)$.

By the lemma, $\Theta(\cdot ; y)$ is indeed a character automorphic function on $\mathbb{C} \cup\{\infty\} \backslash \mathcal{L}$ with simple zeros at $\{\gamma(\zeta)\}_{\gamma \in \Gamma}$ and simple poles at $\left\{\gamma\left(\zeta_{j}\right)\right\}_{\gamma \in \Gamma}$ (and otherwise nonvanishing and finite). By definition,

$$
\Theta(0 ; y)=1
$$


We also define

$$
\tilde{\mathfrak{A}}: \mathbb{G} \rightarrow \Gamma^{*}
$$

by

$$
\tilde{\mathfrak{A}}\left(y_{1}, \ldots, y_{\ell}\right)=\mathfrak{A}\left(y_{1}\right) \cdots \mathfrak{A}\left(y_{\ell}\right)
$$

using the product in $\Gamma^{*}$. Here

$$
\mathbb{G}=G_{1} \times \cdots \times G_{\ell} .
$$

Theorem 5.3. The map $\tilde{\mathfrak{A}}$ of (5.16)-(5.17) is a real analytic homeomorphism of $\ell$-dimensional tori.

Remark. By real analytic functions, we do not mean real-valued but functions of real parameters which are given locally by convergent series of those parameters - they are, of course, $C^{\infty}$.

Proof. By construction, $\Theta(z ; y)$, as a map of $\mathbb{D} \times \cup_{j=1}^{\ell} G_{j}$ to $\mathbb{C}$, is jointly real analytic. Since

$$
\mathfrak{A}(y)(\gamma)=\frac{\Theta(\gamma(0) ; y)}{\Theta(0 ; y)}
$$

$\mathfrak{A}$ and so $\tilde{\mathfrak{A}}$ are real analytic maps.

Suppose $\vec{y}=\left(y_{1}, \ldots, y_{\ell}\right)$ and $\vec{w}=\left(w_{1}, \ldots, w_{\ell}\right)$ lie in $\mathbb{G}$ and $\tilde{\mathfrak{A}}(\vec{y})=$ $\tilde{\mathfrak{A}}(\vec{w})$. Then

$$
f(z)=\prod_{j=1}^{\ell} \frac{\Theta\left(z ; y_{j}\right)}{\Theta\left(z ; w_{j}\right)}
$$

is automorphic since the characters cancel. Hence, there is a unique meromorphic function $F$ on $\mathcal{S}$ so that

$$
f(z)=F\left(\mathbf{x}^{\sharp}(z)\right)
$$

Let $m$ be the number of $j$ with $y_{j} \neq w_{j} . \quad F$ has exactly $m$ poles and $m$ zeros, all simple (the poles/zeros where $y_{j}=w_{j}$ cancel), and so has degree $m \leq \ell$. If $m \neq 0, F \circ \tau \not \equiv F$ since there is a gap with a single simple zero (and if $F \circ \tau=F, F$ has either two zeros or a double zero at a branch point). Thus, if $m \neq 0$, we get a contradiction to Theorem 2.2. It follows that $m=0$, that is, $\vec{y}=\vec{w}$ and $\tilde{\mathfrak{A}}$ is one-one.

Any smooth one-one map between two smooth, orientable compact manifolds of the same dimension has degree \pm 1 , and so is also surjective (see [25, 31, 38, 42, 45, 67] for expositions of degree theory).

We saw above that Theorem 2.2 is the key to the proof of Theorem 5.3. It is also very powerful in connection with Theorem 5.3 as the following theorems show:

Theorem 5.4. Let $f$ be a character automorphic function on $\mathbb{C} \cup\{\infty\} \backslash$ $\mathcal{L}$ with no zeros or poles. Then $f$ is constant. 
Proof. Let $c_{f} \in \Gamma^{*}$ be the character of $f$. By Theorem 5.3, find $\vec{y} \in \mathbb{G}$ with $\tilde{\mathfrak{A}}(\vec{y})=c_{f}$. Let

$$
h(z)=\frac{f(z)}{\prod_{j=1}^{\ell} \Theta\left(z ; y_{j}\right)}
$$

Then $h$ is automorphic, so there is $H$ meromorphic on $\mathcal{S}$ with

$$
h(z)=H\left(\mathbf{x}^{\sharp}(z)\right)
$$

$H$ has degree $m$ where $m=\#\left\{j \mid y_{j} \neq \beta_{j}\right\}$. By Theorem 2.2, $m=0$, that is, $\vec{y}=\left(\beta_{1}, \ldots, \beta_{\ell}\right)$ so $\tilde{\mathfrak{A}}(\vec{y})=1$ and $f$ is automorphic. But then

$$
f(z)=F\left(\mathbf{x}^{\sharp}(z)\right)
$$

with $F$ analytic on $\mathcal{S}$, and therefore $f$ is constant.

Corollary 5.5. Let $\zeta \in \tilde{C}_{j}^{+}$and suppose $h$ is a character automorphic meromorphic function with zeros only at $\{\gamma(\zeta)\}_{\gamma \in \Gamma}$ and poles only at $\left\{\gamma\left(\zeta_{j}\right)\right\}_{\gamma \in \Gamma}$, all simple. If $h(0)=1$, then

$$
h(z)=\Theta\left(z ; \mathbf{x}^{\sharp}(\zeta)\right)
$$

Moreover,

$$
\Theta(\bar{z} ; y)=\overline{\Theta(z ; y)}
$$

and, in particular, $\Theta(\cdot ; y)$ is real and strictly positive on $\mathbb{R}$.

Remark. Thus, $\Theta$ and so $\mathfrak{A}$ are unique.

Proof. Apply Theorem 5.4 to $h(z) / \Theta\left(z ; \mathbf{x}^{\sharp}(\zeta)\right)$.

Definition. By a divisor, we mean a finite subset $\Delta \subset \cup_{j=1}^{\ell} G_{j}$ and the assignment of a nonzero integer $n_{x}$ to each $x \in \Delta$ plus an assignment of an integer $n_{+}$to $\infty_{+}$and

$$
n_{-}=-n_{+}
$$

to $\infty_{-}$. We write the divisor formally as

$$
n_{+} \delta_{\infty_{+}}+n_{-} \delta_{\infty_{-}}+\sum_{x \in \Delta} n_{x} \delta_{x}
$$

Definition. By a special meromorphic function, we mean a meromorphic function on $\mathcal{S}$ so that

(i) All zeros and poles lie in $\left[\cup_{j=1}^{\ell} G_{j}\right] \cup\left\{\infty_{ \pm}\right\}$.

(ii) If $n_{ \pm}$are the order of the zeros and poles at $\infty_{ \pm}\left(n_{ \pm}>0\right.$ means a zero of order $n_{ \pm}, n_{ \pm}<0$ means a pole of order $-n_{ \pm}$), then (5.27) holds. 
Definition. The divisor, $\delta(F)$, of a special meromorphic function $F$ is given by (5.28) where $\Delta$ is the set of zeros and poles of $F$, and $n_{x}$ is the order of the zero/pole at $x$.

Notice that we have chosen a base point $\zeta_{j}$ that depends on which $G_{j}$ the point $y$ lies in. For our later applications, where for each $j$,

$$
\sum_{x \in G_{j}} n_{x}=0
$$

that is very convenient. But when (5.29) does not hold, we will need a factor to move the base point to a fixed point, say $\zeta_{1}$. So we define

$$
\Psi_{j}(z)=\sqrt{\frac{\mathbf{x}(z)-\mathbf{x}\left(\zeta_{j}\right)}{\mathbf{x}(z)-\mathbf{x}\left(\zeta_{1}\right)}}
$$

where we can take special roots by Lemma 5.1, verifying (5.3) as we $\operatorname{did}$ for $(\mathbf{x}(z)-\mathbf{x}(\zeta)) /\left(\mathbf{x}(z)-\mathbf{x}\left(\zeta_{j}\right)\right)$.

We also need to define $\mathfrak{A}_{j}$ to be the character of the character automorphic function $\Psi_{j}$. For $x \in G_{j}$, we let

$$
\mathfrak{A}^{\sharp}(x)=\mathfrak{A}_{j} \mathfrak{A}(x)
$$

Theorem 5.6 (Abel's theorem for special meromorphic functions). $A$ divisor is the divisor of a special meromorphic function $F$ if and only if

$$
\begin{array}{ll}
\text { (a) } & \sum_{x \in \Delta} n_{x}=0 \\
\text { (b) } & \mathfrak{A}(\infty)^{n_{+}} \prod_{x \in \Delta} \mathfrak{A}^{\sharp}(x)^{n_{x}}=1
\end{array}
$$

the identity element of $\Gamma^{*}$.

Remarks. 1. The proof provides an explicit formula for $F$, namely,

$$
F\left(\mathbf{x}^{\sharp}(z)\right)=B(z)^{n_{+}} \prod_{j=1}^{\ell} \prod_{x \in \Delta \cap G_{j}} \Theta(z ; x)^{n_{x}} \Psi_{j}(z)^{n_{x}}
$$

2. Notice that if 5.29 holds, we can drop the $\Psi_{j}$ factors from (5.34) and change $\mathfrak{A}^{\sharp}$ to $\mathfrak{A}$ in $(5.33)$.

Proof. (5.32) is an expression of the constancy of degree, that is, that the number of zeros of $F$ is equal, counting multiplicities, to the number of poles. So really we need to prove, assuming (5.32), that a divisor is a $\delta(F)$ if and only if $(5.33)$ holds.

If the divisor obeys (5.33), then the right side of $(5.34)$ is automorphic, so 5.34 holds for some meromorphic $F$ with the proper zeros 
and poles. Hence, the divisor is a $\delta(F)$. Because of the $\Psi_{j}$ factors, the poles of $\Theta(z ; x)$ at $\zeta_{j}$ are all moved to $\zeta_{1}$ and the poles at $\zeta_{1}$ cancel each other by (5.32).

On the other hand, if $F$ is meromorphic and $\delta(F)$ is its divisor, then letting $f(z)$ be the right side of (5.34),

$$
\frac{F\left(\mathbf{x}^{\sharp}(z)\right)}{f(z)} \equiv h(z)
$$

is character automorphic with no zeros and poles (again, the poles and zeros at $\zeta_{j}$ cancel because of the $\Psi_{j}$ 's and at $\zeta_{1}$ by (5.32). By Theorem 5.4, $h$ is constant, so automorphic. Thus, since $F\left(\mathbf{x}^{\sharp}(z)\right)$ is automorphic, so is $f$, which implies (5.33).

\section{The Isospectral Torus}

Once one has the Abel map and Abel's theorem, the construction of the isospectral torus along the lines pioneered for $\mathrm{KdV}$ [21, 44] is straightforward (see [65, Ch. 5] for an exposition of the original papers 24, 39, 78 ) but in the covering map guise has a more explicit feel. For additional discussions of the isospectral torus, see [7, 11, 28, 29, 71].

Definition. A minimal Herglotz function for $\mathfrak{e}$ is a meromorphic function $m$ on $\mathcal{S}$ with degree precisely $\ell+1$ and which obeys

$$
z \in \mathcal{S}_{+} \cap \mathbb{C}_{+} \Rightarrow \operatorname{Im} m(z)>0
$$

(ii) Near $\infty_{+}$,

$$
m(z)=-\frac{1}{z}+O\left(z^{-2}\right)
$$

(iii) $m(z)$ has a pole at $\infty_{-}$.

In the usual way (see 65]), $m \uparrow \mathcal{S}_{+} \cap \mathbb{C}_{+}$determines a probability measure, $d \mu$, with

$$
m(z)=\int \frac{d \mu(x)}{x-z}
$$

for $z \in \mathcal{S}_{+} \backslash \mathbb{R}$. Moreover, the continuity properties of $m$ as one approaches $\mathfrak{e}$ (and the fact that we will see that all poles of $m$ are simple) implies that

$$
d \mu(x)=w(x) d x+d \mu_{\mathrm{s}}(x)
$$

where $w$ is real analytic on $\mathfrak{e}^{\text {int }}$ and nonvanishing there, and $d \mu_{\mathrm{s}}$ is a pure point measure with pure points only in the open gaps $\cup_{j=1}^{\ell}\left(\beta_{j}, \alpha_{j+1}\right)$ and at most one pure point per gap.

Condition (iii) may seem ad hoc. We mention now that one can show ([65, Ex. 5.13.4]) that if (iii) is dropped, the once-stripped $m$-function, 
$m_{1}$, (i.e., $m(z)=\mathcal{M}\left(a_{1}, b_{1}, m_{1}(z)\right)$ in terms of (1.24)) obeys condition (iii). Thus, the extra possibilities allowed if (iii) is dropped result from taking the Jacobi matrix of a minimal Herglotz function as we have defined it and extending by one row and column, with the "wrong" values of $a_{0}$ or $b_{0}$.

The main elements of the theory are:

(i) The minimal Herglotz functions are in one-one correspondence with $\mathbb{G}$, and so form an $\ell$-dimensional torus, $\mathcal{T}_{\mathfrak{e}}$.

(ii) The correspondence is that the coordinates of $\left(y_{1}, \ldots, y_{\ell}\right) \in \mathbb{G}$ are the positions of $\ell$ of the poles of $m$ - the last pole is at $\infty_{-}$. The zeros are then determined via the Abel map.

(iii) The Abel map "linearizes" coefficient stripping (i.e., the map (1.24) ) since the zeros of $m$ are the poles of the once-stripped $m$-function, $m_{1}$. Explicitly,

$$
\tilde{\mathfrak{A}}: \mathcal{T}_{\mathfrak{e}} \rightarrow \Gamma^{*}
$$

and coefficient stripping corresponds to multiplying by the inverse of the character of $B$.

(iv) The linearization shows that the corresponding Jacobi parameters, $\left\{a_{n}, b_{n}\right\}_{n=1}^{\infty}$, are almost periodic sequences with almost periods given by the harmonic measures $\left\{\rho_{\mathfrak{e}}\left(\left[\alpha_{1}, \beta_{j}\right]\right)\right\}_{j=1}^{\ell}$. In particular, one has periodicity if and only if these numbers are all rational.

(v) The construction provides explicit formulae for $m$ and, thus, $a_{1}, b_{1}$ (and so, via the Abel map, $a_{n}, b_{n}$ ) in terms of theta functions and the logarithmic capacity of $\mathfrak{e}$.

(vi) Uniformly on $\mathcal{T}_{\mathfrak{e}}$, there are bounds on the weight $w$ in (6.4) of the form

$$
C \sqrt{|R(x)|} \leq w(x) \leq D \sqrt{|R(x)|^{-1}}
$$

where $0<C, D<\infty$.

We begin by recalling what one can get without using the covering or Abel maps.

Theorem 6.1. (i) Every minimal Herglotz function has exactly one simple pole in each gap, one at $\infty_{-}$and no others.

(ii) For every choice $\left(y_{1}, \ldots, y_{\ell}\right) \in \mathbb{G}$, there is exactly one minimal Herglotz function with poles exactly at $y_{1}, \ldots, y_{\ell}\left(\right.$ and $\left.\infty_{-}\right)$.

(iii) For every minimal Herglotz function, the once-stripped Herglotz function is also a minimal Herglotz function.

(iv) Every minimal Herglotz function has one zero in each gap, one at $\infty_{+}$and no others. 
Remark. Of course, (i) and (ii) set up a one-one correspondence between $\mathcal{T}_{\mathfrak{e}}$, the set of minimal Herglotz functions, and $\mathbb{G}$. We will often refer to $\mathbb{G}$ as the isospectral torus.

Sketch. (See [65, Ch. 5] for details.) (i) Every minimal degree meromorphic function, $m$, on $\mathcal{S}$ with $m \circ \tau \not \equiv m$ has the form

$$
m(z)=\frac{p(z)+\sqrt{R(z)}}{a(z)}
$$

where $R(z)$ is given by (2.2), and $p, a$ have degree at most $\ell+1$. Since $m$ has a zero at $\infty_{+}, p(z)$ must cancel the leading $O\left(z^{\ell+1}\right)$ term in $\sqrt{R(z)}$ at $\infty_{+}$, so

$$
\operatorname{deg}(p)=\ell+1
$$

Because this cancellation takes place at $\infty_{+}$, it does not at $\infty_{-}$(since $\sqrt{R}$ flips sign but $p$ does not). For $m$ to have a simple pole at $\infty_{-}$, we must have

$$
\operatorname{deg}(a)=\ell
$$

$m$ is real on each $\left[\beta_{j}, \alpha_{j+1}\right]$ so, by analyticity, on the entire gap $G_{j}$. Thus, $m(z)$ is real on $\mathbb{R} \backslash \mathfrak{e}$ on both sheets. Since $\sqrt{R(z)}$ is real on $\mathbb{R} \backslash \mathfrak{e}$, we conclude first that $a$ is real and then that $p$ is real. In particular, on $\overline{\mathcal{S}}_{+} \cap \mathfrak{e}$,

$$
\operatorname{Im} m(x+i 0)=\frac{\operatorname{Im} \sqrt{R(x)}}{a(x)}
$$

Since $R$ has two zeros between bands, Im $\sqrt{R(x)}$ changes sign between successive bands. As $\operatorname{Im} m(x+i 0) \geq 0, a$ must change signs between bands, that is, have an odd number of zeros in each gap. Since, by (6.9), $a$ has only $\ell$ zeros and there are $\ell$ gaps, $a$ has one zero per gap.

If $a$ has a zero at $x_{0} \in\left(\beta_{j}, \alpha_{j+1}\right)$, then

$$
(p+\sqrt{R})-(p-\sqrt{R})=2 \sqrt{R} \neq 0
$$

at $x_{0}$, so on one sheet or the other, $m$ must have a pole. If $a$ has a zero at $x_{0} \in\left\{\beta_{j}, \alpha_{j+1}\right\}$, the numerator is at best $O\left(\left(x-x_{0}\right)^{1 / 2}\right)$ and the denominator is $O\left(\left(x-x_{0}\right)\right)$. So again, $m$ has a pole at $x_{0}$.

Thus, $m$ has at least one pole per gap. So, since $\operatorname{deg}(m)=\ell+1$ and $m$ has a pole at $\infty_{-}, m$ has exactly one simple pole in each gap.

(ii) Write $y_{j}=\left(\pi\left(y_{j}\right), \sigma_{j}\right)$ if $\pi\left(y_{j}\right) \in\left(\beta_{j}, \alpha_{j+1}\right)$ with $\sigma_{j}=1$ (resp. $-1)$ if $y_{j} \in \mathcal{S}_{+}$(resp. $\left.\mathcal{S}_{-}\right)$. Since $a$ has to vanish at $\pi\left(y_{j}\right)$ to get a pole at $y_{j}$, we see that $m$ has a pole at $y_{j}$ and not at $\tau\left(y_{j}\right)$ if and only if $a\left(\pi\left(y_{j}\right)\right)=0$ and

$$
p\left(\pi\left(y_{j}\right)\right)=\sigma_{j} \sqrt{R\left(y_{j}\right)}
$$


If $\pi\left(y_{j}\right) \in\left\{\beta_{j}, \alpha_{j+1}\right\}$, then to avoid a double pole, $p\left(\pi\left(y_{j}\right)\right)=0$, that is, 6.12 still holds (since $\sqrt{R\left(y_{j}\right)}=0$, it does not matter that $\sigma_{j}$ is undefined).

At $\infty_{+}, a(z)$ is $O\left(z^{\ell}\right)$. Thus, for $m(z)$ to vanish at $\infty_{+}$, we must have

$$
p(z)+\sqrt{R(z)}=O\left(z^{\ell-1}\right)
$$

near $\infty_{+}$. Since $\sqrt{R(z)}=O\left(z^{\ell+1}\right)$, 6.13) determines the top two coefficients (with the top one nonzero) and then, by standard polynomial interpolation, the $\ell$ conditions 6.12 determine the remaining $\ell$ coefficients of $p$.

We have thus proven that given $\vec{y}=\left(y_{1}, \ldots, y_{\ell}\right) \in \mathbb{G}$, there is a meromorphic function of degree precisely $\ell+1$ with poles at $y_{1}, \ldots, y_{\ell}$ and $\infty_{-}$, and a zero at $\infty_{+}$. Moreover, it is unique up to a single overall constant - for the above determines $p$ and

$$
a(z)=c \prod_{j=1}^{\ell}\left(z-\pi\left(y_{j}\right)\right)
$$

for some constant $c$.

The fact that $a$ changes sign in each gap shows that the sign of $c$ in (6.14) can be picked so that

$$
\operatorname{Im} m(x+i 0)>0
$$

on all bands in $\mathcal{S}_{+}$. Keeping track of the argument of $\sqrt{R(z)}$ as one crosses a branch point shows that with this choice at each $y_{j} \in \mathcal{S}_{+}$such that $\pi\left(y_{j}\right) \in\left(\beta_{j}, \alpha_{j+1}\right)$ and $\sigma_{j}>0$,

$$
m(z)=\frac{c_{j}}{\pi\left(y_{j}\right)-z}+O(1)
$$

with $c_{j}>0$. Thus, any limit point in the values of $\operatorname{Im} m(z)$ as $z$ approaches $\mathbb{R}$ is nonnegative. Since $\operatorname{Im} m(z) \rightarrow 0$ at $\infty_{+}$, the maximum principle applied to the harmonic function $\operatorname{Im} m(z)$ on $\mathcal{S}_{+} \cap \mathbb{C}_{+}$shows that (6.1) holds.

Any function obeying 6.1 with real boundary values on $\mathbb{R} \backslash \mathfrak{e}$ has (6.2) holding up to a positive constant. We can thus adjust $c$ in (6.14) so that 6.2 holds. We have thus proven that there exists precisely one meromorphic Herglotz function with poles at $y_{1}, \ldots, y_{\ell}$.

(iii) $m$ and the once-stripped function, $m_{1}$, are related by

$$
m(z)=\frac{1}{-z+b_{1}-a_{1}^{2} m_{1}(z)}
$$

where $\left(a_{1}, b_{1}\right)$ are (and can be) chosen so that (6.2) holds for $m_{1}$. It is always true, of course, that $m_{1}$ obeys 6.1 and 6.2. 
6.17) sets up a one-one correspondence between poles of $m_{1}$ in $\mathcal{S}$ $\left\{\infty_{ \pm}\right\}$and zeros of $m$ there. Since $m$ has degree $\ell+1$, it has $\ell+1$ zeros and only a simple zero at $\infty_{+}$by $(6.2)$. Thus, $m$ has precisely $\ell$ zeros in $\mathcal{S} \backslash\left\{\infty_{ \pm}\right\}$.

Therefore, $m_{1}$ has exactly $\ell$ poles on $\mathcal{S} \backslash\left\{\infty_{ \pm}\right\}$and, obviously, no pole at $\infty_{+}$. Since $m(z)$ has a pole at $\infty_{-}, 6.17$ shows that near $\infty_{-}$,

$$
a_{1}^{2} m_{1}(z)=-z+b_{1}+O\left(z^{-1}\right)
$$

that is, $m_{1}$ has a simple pole at $\infty_{-}$. Thus, $m_{1}$ has degree exactly $\ell+1$ and we have proven condition (iii) in the definition of a minimal Herglotz function.

(iv) Since $m_{1}$ has a pole on each $G_{j}$, by (6.17), $m$ has a zero on each $G_{j}$. There is a zero at $\infty_{+}$, and this accounts for all $\ell+1$.

The above construction also lets us prove 6.6.

Theorem 6.2. (i) There are constants, $A, B$, so that uniformly in $\vec{y} \in \mathbb{G}$, we have (with $c$ the constant in (6.14))

$$
A c^{-1} \sqrt{|R(x)|} \leq w_{\vec{y}}(x) \leq B c^{-1} \sqrt{|R(x)|^{-1}}
$$

and the residues $c_{j}$ of 6.16 obey

$$
0 \leq c_{j} \leq B c^{-1}
$$

(ii) Uniformly in $\vec{y} \in \mathbb{G}$, the constant $c$ in (6.14) is bounded and bounded away from zero. Moreover, uniformly in $x \in \mathfrak{e}$ and $\vec{y} \in \mathbb{G}$, (6.6) holds.

Proof. (i) Since $a$ has the form 6.14), we have

$$
\sup _{x \in \mathfrak{e}}|a(x)| \leq c\left(\beta_{\ell+1}-\alpha_{1}\right)^{\ell}
$$

which, given (6.10) and the relation

$$
w_{\vec{y}}(x)=\frac{1}{\pi} \operatorname{Im} m(x+i 0)
$$

implies the first inequality in 6.19.

Next, note that if we fix $x \in \mathfrak{e}$, the distance to the nearest zero of $a$ is at least $\operatorname{dist}(x, \mathbb{R} \backslash \mathfrak{e})$ and the distance to all the other zeros of $a$ at least $\frac{1}{2} \min _{j}\left|\beta_{j}-\alpha_{j}\right|$. Thus, for $x \in \mathfrak{e}$,

$$
|a(x)| \geq c\left(\frac{1}{2} \min _{j}\left|\beta_{j}-\alpha_{j}\right|\right)^{\ell-1} \operatorname{dist}(x, \mathbb{R} \backslash \mathfrak{e})
$$

On the other hand, for $x \in \mathfrak{e}$,

$$
|R(x)| \leq\left(\beta_{\ell+1}-\alpha_{1}\right)^{2 \ell+1} \operatorname{dist}(x, \mathbb{R} \backslash \mathfrak{e})
$$


and

$$
|R(x)| \geq\left(\frac{1}{2} \min _{j}\left|\beta_{j}-\alpha_{j}\right|\right)^{2 \ell+1} \operatorname{dist}(x, \mathbb{R} \backslash \mathfrak{e})
$$

We get the second inequality in 6.19 from $6.23-6.25,6.10$, and 6.22 .

By 6.10 , the residue $c_{j}$ in 6.16 is given by

$$
\begin{aligned}
c_{j} & =2 c^{-1} \sqrt{\left|R\left(y_{j}\right)\right|} \prod_{k \neq j}\left|y_{k}-y_{j}\right|^{-1} \\
& \leq 2 c^{-1}\left(\beta_{\ell+1}-\alpha_{1}\right)^{\ell+1}\left(\min _{j}\left|\beta_{j}-\alpha_{j}\right|\right)^{-\ell}
\end{aligned}
$$

(ii) By 6.19) and 6.20,

$$
\begin{aligned}
A c^{-1} \int_{\mathfrak{e}} \sqrt{|R(x)|} d x & \leq \int_{\mathfrak{e}} w(x) d x+\sum_{j} c_{j} \\
& \leq B c^{-1}\left[\ell+\int_{\mathfrak{e}} \sqrt{|R(x)|^{-1}} d x\right]
\end{aligned}
$$

The total weight of the measure is 1 , so we get the claimed upper and lower bounds on $c$. Given those, 6.19) yields (6.6).

Given $\vec{y} \in \mathbb{G}$, we denote by $m_{\vec{y}}$ the associated minimal Herglotz function. The once-stripped $m$-function is also a minimal Herglotz function and thus corresponds to some $\vec{w} \in \mathbb{G}$. We define a map $U: \mathbb{G} \rightarrow \mathbb{G}$ by

$$
U(\vec{y})=\vec{w}
$$

so that $m_{U(\vec{y})}$ is the once-stripped $m$-function.

Now we can combine Theorem 6.1 with the Abel map:

Theorem 6.3. Suppose $\vec{y}=\left(y_{1}, \ldots, y_{\ell}\right) \in \mathbb{G}$ and let $U$ be defined by 6.29).

(i) With $\tilde{\mathfrak{A}}$ defined in 5.16)-(5.17), we have

$$
\tilde{\mathfrak{A}}(U(\vec{y}))=\tilde{\mathfrak{A}}(\vec{y}) \mathfrak{A}(\infty)^{-1}
$$

(ii) Let

$$
M_{\vec{y}}(z)=-m_{\vec{y}}(\mathbf{x}(z))
$$

Then we have that

$$
M_{\vec{y}}(z)=\frac{B(z)}{\operatorname{cap}(\mathfrak{e})} \prod_{j=1}^{\ell} \frac{\Theta\left(z ; U(\vec{y})_{j}\right)}{\Theta\left(z ; y_{j}\right)}
$$


Remark. Since

$$
m(z)=-\frac{1}{z}-\frac{b_{1}}{z 2}-\frac{a_{1} 2+b_{1} 2}{z 3}+O\left(z^{-4}\right)
$$

6.32 implies an explicit formula for $b_{1}$ and $a_{1}$ in terms of theta functions.

Proof. $M_{\vec{y}}(z)$ is a meromorphic function with divisor

$$
\delta_{\infty_{+}}-\delta_{\infty_{-}}+\sum_{j=1}^{\ell}\left(\delta_{U(\vec{y})_{j}}-\delta_{y_{j}}\right)
$$

so 6.30 is just 5.33 .

By (5.34) (note that 5.29 holds so there are no $\Psi_{j}$ factors), we have 6.32 with $\operatorname{cap}(\mathfrak{e})$ replaced by a constant.

Since

$$
m_{\vec{y}}(\mathbf{x}(z))=-\frac{1}{\mathbf{x}(z)}+O(z 2)=-\frac{z}{x_{\infty}}+O(z 2)
$$

and 4.43) holds, the constant is $\operatorname{cap}(\mathfrak{e})$.

Corollary 6.4. Under the map $\tilde{\mathfrak{A}}$ from $\mathcal{T}_{\mathfrak{e}}$ to $\Gamma^{*},\left\{U^{n}(\vec{y})\right\}_{n=0}^{\infty}$ is mapped to the "equally spaced" orbit $\left\{\tilde{\mathfrak{A}}(\vec{y}) \mathfrak{A}(\infty)^{-n}\right\}_{n=0}^{\infty}$ in $\Gamma^{*}$. In particular,

$$
n \rightarrow m_{U^{n}(\vec{y})} \quad n \rightarrow\left\{a_{n}, b_{n}\right\}
$$

are almost periodic sequences (indeed, real analytic quasiperiodic sequences) with almost periods $\left\{\rho_{\mathfrak{e}}\left(\left[\alpha_{1}, \beta_{j}\right]\right)\right\}_{j=1}^{\ell}$. These sequences are periodic with period $p$ for one point in $\mathcal{T}_{\mathfrak{e}}$ if and only if they are for all points, and that holds if and only if (4.53) holds.

Remark. By a quasiperiodic sequence, $X_{n}$, we mean a sequence given by

$$
X_{n}=x\left(e^{i n \omega_{1}}, \ldots, e^{i n \omega_{k}}\right)
$$

where $x$ is a continuous function on the $k$-torus $(\partial \mathbb{D})^{k}$. It is called real analytic if $x$ is real analytic. $\left(\omega_{1}, \ldots, \omega_{k}\right)$ are called the almost periods.

Proof. 6.30 immediately implies that

$$
\tilde{\mathfrak{A}}\left(U^{n}(\vec{y})\right)=\tilde{\mathfrak{A}}(\vec{y}) \mathfrak{A}(\infty)^{-n}
$$

so the orbit is as claimed.

Realize $\Gamma^{*}$ as $(\partial \mathbb{D})^{\ell}$ by

$$
c \sim\left(c\left(\gamma_{1}\right), \ldots, c\left(\gamma_{\ell}\right)\right)
$$

Then, by 4.44,

$$
\tilde{\mathfrak{A}}\left(U^{n}(\vec{y})\right)_{j}=\tilde{\mathfrak{A}}(\vec{y})_{j} e^{-2 \pi i \rho_{\mathfrak{e}}\left(\left[\alpha_{1}, \beta_{j}\right]\right) n}
$$


which, given that $\tilde{\mathfrak{A}}$ is real analytic and $\Theta(z ; y)$ (and so, $m, a_{1}, b_{1}$ ) are real analytic in the $y$ 's, proves that the sequences (6.35) are almost periodic, indeed, real analytic quasiperiodic.

For the final statement, note that, by (6.37), periodicity for one or for all $\tilde{\mathfrak{A}}(\vec{y})$ is equivalent to $\mathfrak{A}(\infty)^{p}=1$. Now use Corollary 4.5 .

\section{Raw Jost Functions and the Jost Isomorphism}

Recall that if $d \mu$ is a measure on $\mathbb{R}$ of the form (6.4) so that off $[-2,2], d \mu$ only has pure points, $\left\{x_{j}\right\}_{j=1}^{N}$, ( $N$ finite or infinite) with

$$
\sum_{j=1}^{N}\left(\left|x_{j}\right|-2\right)^{1 / 2}<\infty
$$

and so that $w$ obeys a Szegő condition,

$$
\int_{-2}^{2}\left(4-x^{2}\right)^{-1 / 2} \log (w(x))>-\infty
$$

one defines (see $[19$, 36, 50, 60]) the Jost function, $u(z)$, on $\mathbb{D}$ by

$$
u(z)=z B_{\infty}(z) \exp \left(\int \frac{z+e^{i \theta}}{z-e^{i \theta}} \log \left[\frac{\sin \theta}{\operatorname{Im} M\left(e^{i \theta}\right)}\right] \frac{d \theta}{4 \pi}\right)
$$

where $M\left(e^{i \theta}\right)$ is the boundary value of

$$
M(z)=-m\left(z+z^{-1}\right)
$$

(expressible in terms of $w$ via $\left.\operatorname{Im} M\left(e^{i \theta}\right)=\pi w(2 \cos \theta)\right)$ and $B_{\infty}$ is the Blaschke product of $b\left(z, \zeta_{j}\right)$ with $\zeta_{j} \in \mathbb{D}, \zeta_{j}+\zeta_{j}^{-1}=x_{j}$ (by (7.1) and Lemma 4.1, this is a convergent Blaschke product). In this section and the next, we will begin to discuss the analog for elements of the isospectral torus, but in a way that connects up to definitions that work in much greater generality and will be the key to later papers in this series.

Surprisingly, our initial definition will be equal, up to a constant, to an object that is based on the theta function formulae of the last section, and we will find there is a representation of the form (7.3). We regard this as one of the more interesting discoveries in the present paper.

Because there is only one natural choice for the reference measure on $[-2,2]$, it is somewhat obscured that (7.3) involves not only $d \mu$ but a reference measure which in 7.3 is

$$
d \mu_{0}(x)=\frac{1}{2 \pi} \sqrt{4-x^{2}} \chi_{[-2,2]}(x) d x
$$


the measure of the free Jacobi matrix with $a_{n} \equiv 1, b_{n} \equiv 0$. After a change of variables via $x=2 \cos \theta$ and scaling, $\sqrt{4-x^{2}}$ turns into $\sin \theta$, which is where that factor in 7.3 comes from.

When one shifts to multiple gap situations, there is also a reference measure needed. Our eventual choice will be to use a particular point on the isospectral torus - and the next section will explain change of reference measure. In this section, the reference measure will be a different measure on the isospectral torus, so we will call the resulting object the "raw" Jost function.

As a bonus, we will also see that for any point on the isospectral torus,

$$
n \rightarrow \frac{a_{1} \cdots a_{n}}{\operatorname{cap}(\mathfrak{e})^{n}}
$$

is an almost periodic sequence.

Let $\vec{y}_{0}=\left(\beta_{1}, \beta_{2}, \ldots, \beta_{\ell}\right)$ be the point in $\mathbb{G}$ which serves as the base point for our $\Theta$ 's. Given $\vec{y}=\left(y_{1}, \ldots, y_{\ell}\right) \in \mathbb{G}$, we let $\xi_{j}$ be the unique point on $\tilde{C}_{j}^{+}$such that

$$
\mathbf{x}^{\sharp}\left(\xi_{j}\right)=y_{j}, \quad j=1, \ldots, \ell
$$

Moreover, we denote by $w_{\vec{y}}(x)$ the weight of the measure $d \mu_{\vec{y}}$ associated to the $m$-function, $m_{\vec{y}}$, in the isospectral torus.

For each $\vec{y} \in \mathbb{G}$, we define a function on $\mathbb{D}$, the raw Jost function, by

$$
\mathcal{R}(z ; \vec{y})=\prod_{\left\{j|| \xi_{j} \mid<1\right\}} B\left(z, \xi_{j}\right) \exp \left(\frac{1}{4 \pi} \int \frac{e^{i \theta}+z}{e^{i \theta}-z} \log \left[\frac{w_{\overrightarrow{y_{0}}}\left(\mathbf{x}\left(e^{i \theta}\right)\right)}{w_{\vec{y}}\left(\mathbf{x}\left(e^{i \theta}\right)\right)}\right] d \theta\right)
$$

Here we use the estimate $(6.6)$ to be sure that

$$
\int \log \left(w_{\vec{y}}\left(\mathbf{x}\left(e^{i \theta}\right)\right) \frac{d \theta}{2 \pi}>-\infty\right.
$$

for all $\vec{y} \in \mathbb{G}$.

We will call the $\exp (\cdots)$ factor in (7.8), the Szegö part, and the first factor, the Blaschke part. It is easy to see that each is continuous in $\vec{y}$, so $\mathcal{R}(z ; \vec{y})$ is also continuous in $\vec{y}$. The Blaschke factor is only piecewise $C^{1}$ and not $C^{1}$ because whenever $\xi_{j}$ moves from inside $\mathbb{D}$ to outside, a factor disappears. We have $B\left(0, \xi_{j}\right)=\left|\xi_{j}\right|$ and

$$
\xi_{j} \rightarrow \begin{cases}\left|\xi_{j}\right| & \text { if }\left|\xi_{j}\right| \leq 1 \\ 1 & \text { if }\left|\xi_{j}\right| \geq 1\end{cases}
$$

has a discontinuous derivative as $\left|\xi_{j}\right|$ passes from below 1 to above. Nevertheless, we will see below that for many cases (where the harmonic 
measures $\left\{\rho_{\mathfrak{e}}\left(\left[\alpha_{1}, \beta_{j}\right]\right)\right\}_{j=1}^{\ell}$ obey a Diophantine condition $), \vec{y} \mapsto \mathcal{R}(z ; \vec{y})$ is real analytic. It is an interesting open question if this is always true!

We need one more piece of notation. Each $\vec{y} \in \mathbb{G}$ determines a unique $m_{\vec{y}}$ and, thereby, a unique two-sided Jacobi matrix, $\tilde{J}_{\vec{y}}$. Its Jacobi parameters will be denoted $\left\{a_{n}(\vec{y}), b_{n}(\vec{y})\right\}_{n=-\infty}^{\infty}$. Here is the main theorem of this section:

Theorem 7.1. There exists a continuous, everywhere strictly positive function, $\varphi$, on $\mathbb{G}$ so that

$$
\mathcal{R}(z ; \vec{y})=\varphi(\vec{y}) \prod_{j=1}^{\ell} \Theta\left(z ; y_{j}\right)
$$

Moreover, $\varphi$ obeys

$$
\begin{gathered}
\varphi\left(\vec{y}_{0}\right)=1 \\
\frac{a_{1}(\vec{y})}{\operatorname{cap}(\mathfrak{e})}=\frac{\varphi(U(\vec{y}))}{\varphi(\vec{y})}
\end{gathered}
$$

Remarks. 1. In (7.13), $U$ is the map from 6.29).

2. If the harmonic measures $\left\{\rho_{\mathfrak{e}}\left(\left[\alpha_{1}, \beta_{j}\right]\right)\right\}_{j=1}^{\ell}$ are rationally independent, the orbit $\left\{U^{n}\left(\vec{y}_{0}\right)\right\}_{n=0}^{\infty}$ is dense in $\Gamma^{*}$ and $7.12-7.13$ determine $\varphi$ uniquely. In general, $\varphi$ is continuous in $\left\{\alpha_{j}, \beta_{j}\right\}_{j=1}^{\ell}$, so this, in principle, determines it uniquely.

3. It is useful to define, for $\vec{y}=\left(y_{1}, \ldots, y_{\ell}\right) \in \mathbb{G}$,

$$
\tilde{\Theta}(z ; \vec{y})=\prod_{j=1}^{\ell} \Theta\left(z ; y_{j}\right)
$$

We want to note some interesting corollaries before we turn to the proof:

Corollary 7.2. For each $\vec{y} \in \mathbb{G}, \mathcal{R}(z ; \vec{y})$ has a meromorphic continuation to $\mathbb{C} \cup\{\infty\} \backslash \mathcal{L}$ with poles, all simple, only at $\left\{\gamma\left(\zeta_{j}\right)\right\}_{\gamma \in \Gamma, j=1, \ldots, \ell}$, and zeros, all simple, at $\left\{\gamma\left(\xi_{j}\right)\right\}_{\gamma \in \Gamma, j=1, \ldots, \ell}$, where $\zeta_{j}$ and $\xi_{j}$ are given by (5.2) and (7.7), respectively.

Remark. Thus, the Szegö part cancels the poles of $B\left(z, \xi_{j}\right)$ at $\left\{\gamma\left(\bar{\xi}_{j}^{-1}\right)\right\}_{\gamma \in \Gamma}$ for $j$ with $\left|\xi_{j}\right|<1$.

Proof. Obvious from 7.11.

Define the raw Jost isomorphism from $\mathbb{G}$ to $\Gamma^{*}$ by

$$
\mathcal{J}_{r}(\vec{y})=\tilde{\mathfrak{A}}(\vec{y})
$$

By Theorem 5.3, it is a real analytic homeomorphism. The following corollary of Theorem 7.1 is so important, we call it a theorem. 
Theorem 7.3. For each $\vec{y} \in \mathbb{G}, \mathcal{R}(\cdot ; \vec{y})$ is a character automorphic function and its character is $\mathcal{J}_{r}(\vec{y})$. $\mathcal{J}_{r}$ is a real analytic bijection between the isospectral torus and $\Gamma^{*}$.

Remark. The Blaschke part of $\mathcal{R}$ is character automorphic, and we will show directly in Lemma 8.1 that the Szegö part is, too. What is difficult without (7.11) is that the map from $\vec{y}$ to the character of $\mathcal{R}(\cdot ; \vec{y})$ is a bijection. That this map is a bijection will be critical in our proof of Szegö asymptotics in [15].

Proof. Immediate from 7.11 and the definition of $\tilde{\mathfrak{A}}$.

Corollary 7.4. For each $\vec{y} \in \mathbb{G}$, the sequence

$$
n \rightarrow \frac{a_{1}(\vec{y}) \cdots a_{n}(\vec{y})}{\operatorname{cap}(\mathfrak{e})^{n}}
$$

is bounded and almost periodic, also bounded away from 0 . The upper and lower bounds are bounded uniformly in $\vec{y}$.

Remark. That $\left(a_{1} \cdots a_{n}\right)^{1 / n} \rightarrow \operatorname{cap}(\mathfrak{e})$ is a general fact about regular measures, and each $d \mu_{\vec{y}}$ is regular by a theorem of Widom [80] and Van Assche [77] (see [68, 61]). This more subtle result is a special case of a theorem of Widom [81] (see also [3]).

Proof. By (7.13),

$$
\frac{a_{1}(\vec{y}) \cdots a_{n}(\vec{y})}{\operatorname{cap}(\mathfrak{e})^{n}}=\frac{\varphi\left(U^{n}(\vec{y})\right)}{\varphi(\vec{y})}
$$

is given by the values of a continuous function (namely, $\varphi \circ \tilde{\mathfrak{A}}^{-1}$ ) on $\Gamma^{*}$ along the orbit $\tilde{\mathfrak{A}}(\vec{y}) \mathfrak{A}(\infty)^{-n}$. This function is bounded and bounded away from 0 .

One key to the proof of Theorem 7.1 is a nonlocal step-by-step sum rule. Such sum rules began with Killip-Simon [36] for $[-2,2]$, formalized by Simon [57], and one version was found for periodic Jacobi matrices by Damanik-Killip-Simon [18]. The extension of the sum rules to the covering map context, which relies on Beardon's theorem, is a major theme in this paper and especially in the second paper [15] in this series. Here is the version for the isospectral torus:

Theorem 7.5. Let $m_{\vec{y}}$ be the $m$-function for a point $\vec{y} \in \mathbb{G}$. Define for $z \in \mathbb{D}$,

Then for all $z \in \mathbb{D}$,

$$
M_{\vec{y}}(z)=-m_{\vec{y}}(\mathbf{x}(z))
$$

$$
a_{1}(\vec{y}) M_{\vec{y}}(z)=B(z) \frac{\mathcal{R}(z ; U(\vec{y}))}{\mathcal{R}(z ; \vec{y})}
$$


Proof. Let $B^{(\vec{y})}(z)$ be the Blaschke part of $\mathcal{R}$, that is, the Blaschke product in 7.8 . Define

$$
B_{\infty}^{(\vec{y})}(z)=B(z) \frac{B^{(U(\vec{y}))}(z)}{B^{(\vec{y})}(z)}
$$

Then $B_{\infty}^{(\vec{y})}(z)$ has zeros and poles precisely at all the zeros and poles of $M_{\vec{y}}(z)$ inside $\mathbb{D}$, so

$$
h(z)=\frac{a_{1}(\vec{y}) M_{\vec{y}}(z)}{B_{\infty}^{(\vec{y})}(z)}
$$

is analytic and nonvanishing in $\mathbb{D}$.

The same argument that led to (4.84) shows that

$$
z \in \mathcal{F} \Rightarrow\left|\arg \left(B_{\infty}^{(\vec{y})}(z)\right)\right| \leq C
$$

for some constant $C$. Moreover,

$$
z \in \mathcal{F} \Rightarrow\left|\arg \left(M_{\vec{y}}(z)\right)\right| \leq \pi
$$

so

$$
z \in \mathcal{F} \Rightarrow|\arg (h(z))| \leq C_{1}
$$

and, in particular, $\arg (h)$ varies by at most $2 C_{1}$ over $\mathcal{F}$.

$B_{\infty}^{(\vec{y})}$ is character automorphic and $M_{\vec{y}}$ is automorphic, so $h(z)$ is character automorphic. Thus, $\arg (h(z))$ varies by at most $2 C_{1}$ over any $\gamma(\mathcal{F})$, which means that

$$
z \in \mathbb{D}_{k} \Rightarrow|\arg (h(z))| \leq(2 k+1) C_{1}
$$

Thus, by 2.18, for any $r$,

$$
\left\{\theta|| \operatorname{Im} \log \left(h\left(r e^{i \theta}\right)\right) \mid \geq(2 k+1) C_{1}\right\} \subset \partial \mathcal{R}_{k}
$$

So, by Beardon's theorem in the form (3.12), for any $p<\infty$,

$$
\sup _{r} \int\left|\operatorname{Im} \log \left(h\left(r e^{i \theta}\right)\right)\right|^{p} \frac{d \theta}{2 \pi}<\infty
$$

By M. Riesz's theorem (see Rudin [56]),

$$
\log (h) \in \bigcap_{p<\infty} H^{p}(\mathbb{D})
$$

so by the standard representation for $H^{p}$ functions, $p \geq 1$ (see, e.g., [56]), we get

$$
a_{1}(\vec{y}) M_{\vec{y}}(z)=B_{\infty}^{(\vec{y})}(z) \exp \left(\frac{1}{2 \pi} \int \frac{e^{i \theta}+z}{e^{i \theta}-z} \log \left(a_{1}(\vec{y})\left|M_{\vec{y}}\left(e^{i \theta}\right)\right|\right) d \theta\right)
$$


where we used that for a.e. $\theta,\left|B_{\infty}^{(\vec{y})}\left(e^{i \theta}\right)\right|=1$, so

$$
\log \left|h\left(e^{i \theta}\right)\right|=\log \left|M_{\vec{y}}\left(e^{i \theta}\right)\right|
$$

Taking boundary values in

$$
M_{\vec{y}}(z)^{-1}=\mathbf{x}(z)-b_{1}-a_{1}^{2} M_{U(\vec{y})}(z)
$$

we see that

or

$$
\frac{\operatorname{Im} M_{\vec{y}}\left(e^{i \theta}\right)}{\left|M_{\vec{y}}\left(e^{i \theta}\right)\right|^{2}}=a_{1}^{2} \operatorname{Im} M_{U(\vec{y})}\left(e^{i \theta}\right)
$$

$$
\begin{aligned}
\log \left(a_{1}(\vec{y})\left|M_{\vec{y}}\left(e^{i \theta}\right)\right|\right) & =\frac{1}{2} \log \left[\frac{\operatorname{Im} M_{\vec{y}}\left(e^{i \theta}\right)}{\operatorname{Im} M_{U(\vec{y})}\left(e^{i \theta}\right)}\right] \\
& =\frac{1}{2} \log \left[\frac{\operatorname{Im} M_{\vec{y}}\left(e^{i \theta}\right)}{\operatorname{Im} M_{\overrightarrow{y_{0}}}\left(e^{i \theta}\right)}\right]-\frac{1}{2} \log \left[\frac{\operatorname{Im} M_{U(\vec{y})}\left(e^{i \theta}\right)}{\operatorname{Im} M_{\overrightarrow{y_{0}}}\left(e^{i \theta}\right)}\right]
\end{aligned}
$$

Since $\operatorname{Im} M_{\vec{y}}\left(e^{i \theta}\right)=\pi w_{\vec{y}}\left(\mathbf{x}\left(e^{i \theta}\right)\right)$, (7.29) plus (7.34) and the definition (7.3) implies (7.19).

Corollary 7.6. For any $\vec{y} \in \mathbb{G}$, we have

$$
\frac{\mathcal{R}(z ; U(\vec{y}))}{\tilde{\Theta}(z ; U(\vec{y}))}=\frac{a_{1}(\vec{y})}{\operatorname{cap}(\mathfrak{e})} \frac{\mathcal{R}(z ; \vec{y})}{\tilde{\Theta}(z ; \vec{y})}
$$

Proof. Immediate from 6.32 and 7.19.

Proof of Theorem 7.1. For $\vec{y}_{0}$, we have

$$
\tilde{\Theta}\left(z ; \vec{y}_{0}\right)=\mathcal{R}\left(z ; \vec{y}_{0}\right)=1
$$

Thus, by (7.35), we have 7.11 for $\vec{y}=U^{n}\left(\vec{y}_{0}\right)$ with

$$
\varphi\left(U^{n}\left(\vec{y}_{0}\right)\right)=\frac{a_{1}\left(\vec{y}_{0}\right) \cdots a_{n}\left(\vec{y}_{0}\right)}{\operatorname{cap}(\mathfrak{e})^{n}}
$$

since

$$
a_{1}\left(U^{n}\left(\vec{y}_{0}\right)\right)=a_{n+1}\left(\vec{y}_{0}\right)
$$

Thus, (7.13) also holds for $\vec{y}=U^{n}\left(\vec{y}_{0}\right)$.

Suppose now $\left\{\alpha_{j}, \beta_{j}\right\}_{j=1}^{\ell+1}$ are such that $\left\{\rho_{\mathfrak{e}}\left(\left[\alpha_{1}, \beta_{j}\right]\right)\right\}_{j=1}^{\ell}$ are rationally independent. Then $S_{0} \equiv\left\{U^{n}\left(\vec{y}_{0}\right)\right\}$ is dense in the isospectral torus. Define

$$
\varphi(\vec{y}) \equiv \frac{\mathcal{R}(z=0 ; \vec{y})}{\Theta(z=0 ; \vec{y})}
$$

Since $\mathcal{R}$ is continuous in $\vec{y}$, so is $\varphi$. As 7.11 holds on the dense set $S_{0}$ and both sides are continuous, (7.11) holds for all $\vec{y} \in \mathbb{G}$. Similarly, both sides of (7.13) are continuous and as (7.13) holds on $S_{0}$, it holds in general. 
To handle general $\left\{\alpha_{j}, \beta_{j}\right\}_{j=1}^{\ell+1}$, we just repeat the continuity argument "at a higher level." Fix $\alpha_{1}, \ldots, \alpha_{\ell+1}$ and $\beta_{\ell+1}$ and vary $\left(\beta_{1}, \ldots, \beta_{\ell}\right)$. By a theorem of Totik [72], the map $\left(\beta_{1}, \ldots, \beta_{\ell}\right) \rightarrow\left\{\rho_{\mathfrak{e}}\left(\left[\alpha_{j}, \beta_{j}\right]\right)\right\}_{j=1}^{\ell}$ is a $C^{\infty}$ local bijection so the set of $\beta$ 's with rationally independent $\left\{\rho_{\mathfrak{e}}\left(\left[\alpha_{j}, \beta_{j}\right]\right)\right\}_{j=1}^{\ell}$ is dense. By conveniently labelling $\mathbb{G}$ (say, measure angles in $\tilde{C}_{j}^{+}$about its center starting at the point closest to 0 ) in a way that is independent of $\left\{\alpha_{j}, \beta_{j}\right\}_{j=1}^{\ell+1}$, all objects, that is, $\mathcal{R}(z ; \vec{y})$ and $\Theta(z ; \vec{y})$, are continuous in $\left(\beta_{1}, \ldots, \beta_{\ell}\right)$ according to Theorem 2.1. Therefore, by repeating the above argument, we get 7.11 and $(7.13$ by continuity.

Finally, we want to note that sometimes $\varphi$ and so $\mathcal{R}$ are real analytic in $\vec{y}$. We say that $\mathfrak{e}$ obeys a Diophantine condition if there are a constant, $C$, and an integer, $k$, so that for $\left(n_{1}, \ldots, n_{\ell}\right) \neq(0, \ldots, 0)$ in $\mathbb{Z}^{\ell}$

$$
\left|\sum_{j=1}^{\ell} n_{j} \rho_{\mathfrak{e}}\left(\left[\alpha_{1}, \beta_{j}\right]\right)\right| \geq C(1+|n|)^{-k}
$$

As is well known, given Totik's theorem quoted above, Lebesgue a.e. $\left\{\alpha_{j}, \beta_{j}\right\}_{j=1}^{\ell+1}$ lead to a Diophantine $\mathfrak{e}$.

Theorem 7.7. If $\mathfrak{e}$ is Diophantine, the function $\varphi$ of 7.11 is real analytic in $\vec{y}$ and thus, $\mathcal{R}(z ; \vec{y})$ is real analytic in $\vec{y}$.

Proof. $\Theta$ is real analytic in $y$, so by 7.11 , the statement about $\varphi$ implies that for $\mathcal{R}$.

Let

$$
L(\vec{y})=\log \left(\frac{a_{1}(\vec{y})}{\operatorname{cap}(\mathfrak{e})}\right)
$$

Then

$$
S(\vec{y}) \equiv \log (\varphi(\vec{y}))
$$

obeys

$$
S(U(\vec{y}))-S(\vec{y})=L(\vec{y})
$$

Since $L$ is real analytic on the torus, its Fourier coefficients, $l_{\left(n_{1}, \ldots, n_{\ell}\right)}$, obey

$$
\left|l_{\left(n_{1}, \ldots, n_{\ell}\right)}\right| \leq C e^{-D|n|}
$$

for some $C, D>0$. By 7.42 , the Fourier coefficients $s_{\left(n_{1}, \ldots, n_{\ell}\right)}$ for $S$ obey

where

$$
\left(e^{i n \cdot \omega}-1\right) s_{\left(n_{1}, \ldots, n_{\ell}\right)}=l_{\left(n_{1}, \ldots, n_{\ell}\right)}
$$

$$
n \cdot \omega=\sum_{j=1}^{\ell} n_{j} \rho_{\mathfrak{e}}\left(\left[\alpha_{1}, \beta_{j}\right]\right)
$$


(7.44) implies $\ell_{0}=0$ and $\left.(7.43)-7.44\right)$ together with 7.39 imply that for any $\varepsilon>0$,

$$
\left|s_{\left(n_{1}, \ldots, n_{\ell}\right)}\right| \leq C_{\varepsilon} e^{-(D-\varepsilon)|n|}
$$

This implies that $S(\vec{y})$ and so $\varphi(\vec{y})=e^{S(\vec{y})}$ are real analytic.

\section{Change of Reference Measure in Jost Functions}

As is well known, OPRL obey a difference equation, (1.6). We will also be interested in other solutions. Since we have labelled the Jacobi parameters starting at $n=1$, it will be useful to label solutions that way too, that is, to look for solutions of

$$
a_{n} u_{n+1}+\left(b_{n}-z\right) u_{n}+a_{n-1} u_{n-1}=0
$$

for $n=1,2, \ldots$ where $a_{0}$ is often picked to be 1 , but in the isospectral torus will be the natural two-sided almost periodic $a_{0}$. Note that

$$
u_{n}=p_{n-1}(z)
$$

$n=1,2, \ldots$ is a solution of 8.1 with $p_{-1}=0$ (so $a_{0}$ is unimportant).

For any bounded Jacobi matrix, $J$, and $z \in \mathbb{C}_{+}$, there is a unique solution of (8.1) which is $\ell^{2}$ at infinity, unique up to an overall constant (see, e.g., 65]). One natural choice is the Weyl solution,

$$
W_{n}(z)=\left\langle\delta_{n},(z-J)^{-1} \delta_{1}\right\rangle
$$

It obeys 8.1 for $n=2,3, \ldots$ At $n=1$, one has

$$
a_{1} W_{2}+\left(b_{1}-z\right) W_{1}=-1
$$

(since $\left.(J-z)(J-z)^{-1} \delta_{1}=\delta_{1}\right)$, and so 8.1 holds at $n=1$ if we define

$$
W_{0}=a_{0}^{-1}
$$

One defect of this solution is that, of course, $W_{n}(z)$ has a pole at each discrete eigenvalue of $J$. To get around this, Jost had the idea (for continuum Schrödinger operators) of multiplying $W_{n}(z)$ by a $z$-dependent constant $u_{0}(z)$ with a zero at the eigenvalues. Then $u_{0}(z) W_{n}(z)$ can have a removable singularity at such $z$ 's. Our raw Jost functions have zeros at the $\xi_{j}$ 's, so when we look at solutions in the next two sections, the poles in the Weyl solutions will be cancelled by these zeros. But we are going to want to continue these solutions up to the bands also, and our raw Jost functions have poles at the $\zeta_{j}$ 's. These poles are not intrinsic to the $y$ 's, but come from the $w_{\vec{y}_{0}}$ term in (7.8). We thus want to consider modifying that. 
Definition. By the Szegő class for $\mathfrak{e}$, we mean the set of measures $d \mu$ of the form (6.4) where $w$ is supported on $\mathfrak{e}$ and obeys the Szego" condition (4.56) (with $f=w$ ), and so that outside $\mathfrak{e}, d \mu_{\mathrm{s}}$ only has countably many pure points $\left\{x_{j}\right\}_{j=1}^{N}$ obeying a Blaschke condition of the form 4.76).

We are now prepared to define the Jost functions with reference measure $d \mu_{1}$.

Definition. Suppose $d \mu, d \mu_{1}$ are two measures in the Szegö class for $\mathfrak{e}$. The Jost function, $\mathcal{J}\left(z ; \mu, \mu_{1}\right)$, with reference measure $d \mu_{1}$, is the meromorphic function on $\mathbb{D}$ defined by

$$
\mathcal{J}\left(z ; \mu, \mu_{1}\right)=\frac{\prod_{j=1}^{N} B\left(z, z_{j}\right)}{\prod_{j=1}^{N_{1}} B\left(z, z_{j}^{(1)}\right)} \exp \left(\frac{1}{4 \pi} \int \frac{e^{i \theta}+z}{e^{i \theta}-z} \log \left[\frac{w_{1}\left(\mathbf{x}\left(e^{i \theta}\right)\right)}{w\left(\mathbf{x}\left(e^{i \theta}\right)\right)}\right] d \theta\right)
$$

where $\left\{z_{j}\right\}_{j=1}^{N}$ are the points in $\mathcal{F}$ with $\mathbf{x}\left(z_{j}\right)=x_{j}$ (and similarly for $z_{j}^{(1)}$ and $\left.x_{j}^{(1)}\right)$.

Notice that, by Proposition 4.8 and condition (4.76), the Blaschke products converge. By Corollary 4.6 and (4.56), the integral in (8.6) converges. Notice that if $d \mu_{1}$ has pure points in the gaps, then $\mathrm{d}$ has poles in $\mathbb{D}$. For this reason, we will normally consider only $d \mu_{1}$ 's with no such pure points, but since we want to consider the entire isospectral torus later in this section, we allow for the possibility.

We are heading towards proving that $\mathcal{J}$ is character automorphic. The key is

Lemma 8.1. Suppose $f$ is a real-valued function on $\partial \mathbb{D}$ so that

$$
\int\left|f\left(e^{i \theta}\right)\right| \frac{d \theta}{2 \pi}<\infty
$$

and for all $\gamma \in \Gamma$,

$$
f\left(\gamma\left(e^{i \theta}\right)\right)=f\left(e^{i \theta}\right)
$$

Define for $z \in \mathbb{D}$,

$$
S_{f}(z)=\exp \left(\int \frac{e^{i \theta}+z}{e^{i \theta}-z} f\left(e^{i \theta}\right) \frac{d \theta}{2 \pi}\right)
$$

Then $S_{f}$ is character automorphic.

Remark. If

$$
f\left(e^{i \theta}\right)=\log \left[\frac{\operatorname{Im} w_{\vec{y} 0}\left(\mathbf{x}\left(e^{i \theta}\right)\right)}{\operatorname{Im} w_{\vec{y}}\left(\mathbf{x}\left(e^{i \theta}\right)\right)}\right]
$$


where $\vec{y}$ has no points in $\mathbb{D}$, then $S_{f}$ is a raw Jost function which, if $\vec{y} \neq \vec{y}_{0}$, is not automorphic but only character automorphic (by Theorem 7.3). Thus, $S_{f}$ may have a nontrivial character.

Proof. Suppose first there is $a>0$ so that

$$
-a \leq f\left(e^{i \theta}\right) \leq a
$$

for all $\theta$. Then, since

$$
\int \operatorname{Re}\left(\frac{e^{i \theta}+z}{e^{i \theta}-z}\right) \frac{d \theta}{2 \pi}=1
$$

with positive integrand, we have

$$
e^{-a} \leq\left|S_{f}(z)\right| \leq e^{a}
$$

In particular, if $\gamma \in \Gamma$, then

$$
h(z) \equiv \frac{S_{f}(\gamma(z))}{S_{f}(z)}
$$

is analytic and

$$
e^{-2 a} \leq|h(z)| \leq e^{2 a}
$$

so one can define $\log (h)$ on $\mathbb{D}$ and it belongs to $H^{\infty}(\mathbb{D})$.

By (8.9), for Lebesgue a.e. $\theta$,

$$
\lim _{r \uparrow 1}\left|S_{f}\left(r e^{i \theta}\right)\right|=e^{f\left(e^{i \theta}\right)}
$$

Since $\gamma$ maps $\mathbb{D}$ to $\mathbb{D}$ and boundary values are nontangential limits, for Lebesgue a.e. $\theta$,

$$
\lim _{r \uparrow 1}\left|S_{f}\left(\gamma\left(r e^{i \theta}\right)\right)\right|=e^{f\left(\gamma\left(e^{i \theta}\right)\right)}=e^{f\left(e^{i \theta}\right)}
$$

by the hypothesis 8.8 .

It follows that for a.e. $\theta$,

$$
\lim _{r \uparrow 1} \operatorname{Re}\left(\log \left(h\left(r e^{i \theta}\right)\right)\right)=\lim _{r \uparrow 1} \log \left|h\left(r e^{i \theta}\right)\right|=0
$$

Since $\log |h|$ is a bounded harmonic function, $\operatorname{Re} \log (h(z))=0$, so $\log (h(z))=i \psi_{\gamma}$ for some real $\psi_{\gamma}$, that is,

$$
S_{f}\left(\gamma\left(e^{i \theta}\right)\right)=e^{i \psi_{\gamma}} S_{f}\left(e^{i \theta}\right)
$$

As usual, this implies that $\gamma \rightarrow e^{i \psi_{\gamma}}$ is a character, and so $S_{f}$ is character automorphic.

If $f$ does not obey (8.11), it is easy to write it as an $L^{1}$ limit of functions that do. Thus, $S_{f}$ is a uniform (on compact subsets of $\mathbb{D}$ ) limit of character automorphic functions. By the compactness of $\Gamma^{*}$, it is easy to see that any such limit is character automorphic. 
Theorem 8.2. For any $d \mu, d \mu_{1}$ in the Szegö class, $\mathcal{J}\left(z ; \mu, \mu_{1}\right)$ is a character automorphic function.

Remark. For $d \mu, d \mu_{1}$ both in the isospectral torus, this follows from Theorem 7.3 and Theorem 8.3 below.

Proof. Immediate from (8.6), 4.20), and Lemma 8.1.

Theorem 8.3 (Change of reference measure in $\mathcal{J}$ ). Let $d \mu, d \mu_{1}, d \mu_{2}$ be three measures in the Szegö class. Then for all $z \in \mathbb{D}$,

$$
\mathcal{J}\left(z ; \mu, \mu_{1}\right)=\frac{\mathcal{\partial}\left(z ; \mu, \mu_{2}\right)}{\mathcal{J}\left(z ; \mu_{1}, \mu_{2}\right)}
$$

In particular, for $\vec{y}, \vec{y}_{1} \in \mathbb{G}$, we have

$$
\mathcal{J}\left(z ; \mu_{\vec{y}}, \mu_{\vec{y}_{1}}\right)=\frac{\mathcal{R}(z ; \vec{y})}{\mathcal{R}\left(z ; \vec{y}_{1}\right)}
$$

Remark. By "all $z \in \mathbb{D}$," we either mean except for the discrete set of poles and zeros or else in the sense of meromorphic functions.

Proof. In (8.6), the Blaschke products and the $\log \left[w_{1}\right], \log [w]$ factors can be separated out and cancelled and recombined.

Corollary 8.4. For any Szegö class measure, $d \mu_{1}$, the character $\mathcal{J}_{\mu_{1}}(\vec{y})$ of $\mathcal{J}\left(z ; \mu_{\vec{y}}, \mu_{1}\right)$ defines a real analytic bijection of $\mathbb{G}$ and $\Gamma^{*}$.

Proof. By (8.20),

$$
\mathcal{J}_{\mu_{1}}(\vec{y})=\mathcal{J}_{r}(\vec{y}) \mathcal{J}_{\mu_{1}}\left(\vec{y}_{0}\right)
$$

Since $\vec{y} \mapsto \mathcal{J}_{r}(\vec{y})$ is a bijection of $\mathbb{G}$ and $\Gamma^{*}$ (by Theorem 7.3) and $\chi \mapsto \chi \mathcal{J}_{\mu_{1}}\left(\vec{y}_{1}\right)$ is a bijection of $\Gamma^{*}, \vec{y} \mapsto \mathcal{J}_{\mu_{1}}(\vec{y})$ is a bijection.

Corollary 8.5. For any $\vec{y}, \vec{y}_{1} \in \mathbb{G}$,

$$
\mathcal{J}\left(z ; \mu_{\vec{y}}, \mu_{\vec{y}_{1}}\right)=\varphi(\vec{y}) \varphi\left(\vec{y}_{1}\right)^{-1} \frac{\tilde{\Theta}(z ; \vec{y})}{\tilde{\Theta}\left(z ; \vec{y}_{1}\right)}
$$

where $\varphi$ is the function of Theorem 7.1 and $\tilde{\Theta}$ is given by (7.14).

Proof. Immediate from (7.11) and (8.21).

Since we want to make the poles of $\mathcal{J}$ as far from $\mathcal{S}_{+}$as possible, we define $\vec{w}$ to be the point on $\mathbb{G}$ whose coordinates $\left(w_{1}, \ldots, w_{\ell}\right)$ have points $\tilde{\zeta}_{1}, \ldots, \tilde{\zeta}_{\ell}$ in $\tilde{C}_{j}^{+}$with $\mathbf{x}^{\sharp}\left(\tilde{\zeta}_{j}\right)=w_{j}$ and

$$
\left|\tilde{\zeta}_{j}\right|=\max _{\zeta \in \tilde{C}_{j}^{+}}|\zeta|
$$


Definition. Let $d \nu$ be the measure in $\mathcal{T}_{\mathfrak{e}}$ associated to $\vec{w}$. For any $d \mu$ in the Szegő class, we define the Jost function, $u(z ; \mu)$, by

$$
u(z ; \mu)=\mathcal{J}(z ; \mu, \nu)
$$

For $\vec{y} \in \mathbb{G}$, we use $u(z ; \vec{y})$ for $u\left(z ; \mu_{\vec{y}}\right)$.

$u(z ; \mu)$ will play a major role in the later papers of this series. $u(z ; \vec{y})$ will concern us in the rest of this paper. We begin by noting

Theorem 8.6. There is a neighborhood, $N$, of $\overline{\mathcal{F}}$ (closure and neighborhood in $\mathbb{C}$ ) so that each $u(\cdot ; \vec{y})$ is analytic in $N$ and $u$ is uniformly bounded on $N$ and in $\vec{y} \in \mathbb{G}$.

Proof. Obvious from 8.23), which says that

$$
u(z ; \vec{y})=\varphi(\vec{y}) \varphi(\vec{w})^{-1} \frac{\tilde{\Theta}(z ; \vec{y})}{\tilde{\Theta}(z ; \vec{w})}
$$

and the fact that the ratio of $\tilde{\Theta}$ 's has poles only at $\left\{\gamma\left(\tilde{\zeta}_{j}\right)\right\}_{\gamma \in \Gamma, j=1, \ldots, \ell}$.

Proposition 8.7. For $z \in \mathbb{R}, u(z ; \vec{y})>0$.

Proof. Follows from (8.26) and the facts that $\varphi$ is positive and that $\Theta$ is positive on $\mathbb{R}$.

\section{Jost Solutions}

One big benefit of the covering map formalism is that it provides explicit information about solutions of (8.1) for $J$ in the isospectral torus and, thereby, of ground states, spectral theorist's Green's function, etc. We begin by moving the Weyl solutions, 8.3), to $\mathbb{D}$ :

Definition. For $z \in \mathbb{D}$, the Weyl solution is defined by

$$
W_{n}(z)=\left\langle\delta_{n},(\mathbf{x}(z)-J)^{-1} \delta_{1}\right\rangle
$$

For the case $\mathfrak{e}=[-2,2]$, this function is studied in Section 13.9 of 60]. The proof of Proposition 13.9.3 of [60] is purely algebraic and immediately extends to our context:

Theorem 9.1. Suppose $J$ is the Jacobi matrix of any OPRL with $\sigma_{\text {ess }}(J)=\mathfrak{e}$ and define $M$ by

$$
M(z)=-m(\mathbf{x}(z))
$$

for $z \in \mathbb{D}$. Let $J^{(n)}$ be the $n$-times stripped Jacobi matrix, that is,

$$
a_{j}^{(n)}=a_{n+j} \quad b_{j}^{(n)}=b_{n+j}
$$


and denote by $M^{(n)}$ its $m$-function on $\mathbb{D}$. Then

$$
W_{n}(z)=M(z)\left(a_{1} M^{(1)}(z)\right) \cdots\left(a_{n-1} M^{(n-1)}(z)\right)
$$

Definition. Suppose $d \mu$ lie in the Szegö class. Then the Jost solution is defined for $z \in \mathbb{D}$ by

$$
u_{n}(z)=u(z ; \mu) W_{n}(z)
$$

We focus here on the case $d \mu=d \mu_{\vec{y}}$ for $\vec{y} \in \mathbb{G}$, in which case we use the notation $u_{n}(z ; \vec{y})$.

Theorem 9.2. For $n \geq 1$, we have

$$
u_{n}(z ; \vec{y})=a_{n}^{-1} B(z)^{n} u\left(z ; U^{n}(\vec{y})\right)
$$

If (9.6) is used to define $u_{n}$ for all $n \in \mathbb{Z}$, then

(i) $u_{n}$ solves (8.1).

(ii) There is a neighborhood, $N$, of $\overline{\mathcal{F}}$ so that $u_{n}(z ; \vec{y})$ has an analytic continuation to $N$ and is real analytic in $\vec{y}$.

(iii) $B(z)^{-n} u_{n}(z ; \vec{y})$ is almost periodic in $n$. Indeed, uniformly for $z \in$ $N$ and $\vec{y} \in \mathbb{G}$, it is real analytic quasiperiodic.

Proof. By 7.19,

$$
a_{j+1} M^{(j)}(z)=B(z) \frac{u\left(z ; U^{j+1}(\vec{y})\right)}{u\left(z ; U^{j}(\vec{y})\right)}
$$

Thus, by (9.4),

$$
\begin{aligned}
a_{n} W_{n}(z) & =\prod_{j=0}^{n-1} a_{j+1} M^{(j)}(z) \\
& =B(z)^{n} \frac{u\left(z ; U^{n}(\vec{y})\right)}{u(z ; \vec{y})}
\end{aligned}
$$

which is 9.6 .

By (9.6), we have

$$
a_{n+j} u_{n+j}(z ; \vec{y})=a_{n} B(z)^{j} u_{n}\left(z ; U^{j}(\vec{y})\right)
$$

for all $n, j \in \mathbb{Z}$. Since $W_{n}$ solves (8.1) for $n \geq 1, u_{n}$ does also, and then by (9.9), $u_{n}$ solves 8.1 for all $n \in \mathbb{Z}$. (ii) is immediate from Theorem 8.6. (iii) is then immediate from $(9.6)$ and the fact that under $\tilde{\mathfrak{A}}, U$ is transformed to multiplication $\left(\right.$ by $\mathfrak{A}(\infty)^{-1}$ ) on $\Gamma^{*}$.

For $x \in \mathfrak{e}$, define $u^{+}$by picking $\mathbf{z}(x) \in \overline{\mathcal{F}}$ with $\operatorname{Im} \mathbf{z} \geq 0$, so that $\mathbf{x}(\mathbf{z}(x))=x$, and letting

$$
u_{n}^{+}(x ; \vec{y})=u_{n}(\mathbf{z}(x) ; \vec{y})
$$


We also let

$$
u_{n}^{-}(x ; \vec{y})=\overline{u_{n}^{+}(x ; \vec{y})}=u_{n}(\overline{\mathbf{z}(x)} ; \vec{y})
$$

Theorem 9.3. Define the Wronskian, $\operatorname{Wr}(f, g)$, of two solutions of (8.1) by

$$
\mathrm{Wr}(f, g)=a_{n}\left(f_{n+1} g_{n}-f_{n} g_{n+1}\right)
$$

(which is $n$-independent). Then, if $x=\mathbf{x}(\mathbf{z}(x))$ and

$$
\operatorname{Im} \mathbf{z}>0 \quad x \in \mathfrak{e}^{\mathrm{int}}
$$

we have

$$
\operatorname{Wr}\left(u_{.}^{+}(x ; \vec{y}), u_{.}^{-}(x ; \vec{y})\right)=2 \pi i|u(\mathbf{z}(x) ; \vec{y})|^{2} w_{\vec{y}}(x)
$$

where $w_{\vec{y}}$ is the weight in the spectral measure, $d \mu_{\vec{y}}$.

Proof. Since $W_{0}=a_{0}^{-1}$,

$$
\begin{aligned}
\operatorname{Wr}(\bar{W}, W) & =a_{0}\left(\bar{W}_{1} a_{0}^{-1}-W_{1} a_{0}^{-1}\right) \\
& =-2 i \operatorname{Im} W_{1}
\end{aligned}
$$

Taking into account that

$$
\frac{1}{\pi} \operatorname{Im} m\left(x_{0}+i 0\right)=w\left(x_{0}\right)
$$

and

$$
\operatorname{Wr}(\bar{c} \bar{f}, c f)=|c|^{2} \operatorname{Wr}(\bar{f}, f)
$$

we get $(9.14)$.

Recall ([65, Ch. 3]) that the transfer matrix, $T_{n}(z)$, for a Jacobi matrix updates solutions of (8.1) via

$$
\left(\begin{array}{c}
u_{n+1} \\
a_{n} u_{n}
\end{array}\right)=T_{n}(z)\left(\begin{array}{c}
u_{1} \\
a_{0} u_{0}
\end{array}\right)
$$

and is given by

$$
T_{n}(z)=\left(\begin{array}{cc}
p_{n}(z) & -q_{n}(z) \\
a_{n} p_{n-1}(z) & -a_{n} q_{n-1}(z)
\end{array}\right)
$$

where $q_{n}$ are the second kind polynomials. As usual, if we want to indicate the underlying point in the isospectral torus, we write $T_{n}(z ; \vec{y})$.

Theorem 9.4. There is a constant $C$ so that uniformly for $\vec{y} \in \mathbb{G}$ and $x \in \mathfrak{e}^{\text {int }}$,

$$
\left\|T_{n}(x ; \vec{y})\right\| \leq C \operatorname{dist}(x, \mathbb{R} \backslash \mathfrak{e})^{-1 / 2}
$$

Remark. This result is used in Proposition 7.2 of [10]. 
Proof. Let $U_{n}(x ; \vec{y})$ be the matrix

$$
U_{n}(x ; \vec{y})=\left(\begin{array}{cc}
u_{n+1}^{+}(x ; \vec{y}) & u_{n+1}^{-}(x ; \vec{y}) \\
a_{n} u_{n}^{+}(x ; \vec{y}) & a_{n} u_{n}^{-}(x ; \vec{y})
\end{array}\right)
$$

Then

$$
T_{n}(x ; \vec{y}) U_{0}(x ; \vec{y})=U_{n}(x ; \vec{y})
$$

SO

$$
T_{n}(x ; \vec{y})=U_{n}(x ; \vec{y}) U_{0}(x ; \vec{y})^{-1}
$$

and, since $2 \times 2$ matrices obey $\left\|C^{-1}\right\|=|\operatorname{det}(C)|^{-1}\|C\|$,

$$
\left\|T_{n}(x ; \vec{y})\right\| \leq\left|\operatorname{det}\left(U_{0}(x ; \vec{y})\right)\right|^{-1}\left\|U_{0}(x ; \vec{y})\right\|\left\|U_{n}(x ; \vec{y})\right\|
$$

As $u_{n}$ is uniformly bounded in $n, \vec{y}$, and $x \in \mathfrak{e}$, and $\operatorname{det}\left(U_{0}\right)$ is the Wronskian, by (9.14),

$$
\left\|T_{n}(x ; \vec{y})\right\| \leq C w_{\vec{y}}(x)^{-1}
$$

which yields (9.20, given 6.6.

Corollary 9.5. Uniformly in $n, x \in \mathfrak{e}^{\text {int }}$, and $\vec{y} \in \mathbb{G}$,

$$
C_{1} \operatorname{dist}(x, \mathbb{R} \backslash \mathfrak{e}) \leq\left|p_{n}(x)\right|^{2}+\left|p_{n-1}(x)\right|^{2} \leq C_{2} \operatorname{dist}(x, \mathbb{R} \backslash \mathfrak{e})^{-1}
$$

for suitable constants $C_{1}$ and $C_{2}$.

Proof. Immediate from (9.19), $\operatorname{det}\left(T_{n}\right)=1$ (so $\left.\left\|T_{n}^{-1}\right\|=\left\|T_{n}\right\|\right)$, and (9.20), recalling that the $a_{n}$ 's are bounded and bounded away from 0 .

Next, we look at band edges where $\left\|T_{n}\right\|$ can diverge. We begin with a critical fact about the outer edges:

Theorem 9.6. There are positive, finite constants $C_{1}$ and $C_{2}$, so that uniformly in $n$ and $\vec{y}$,

$$
\begin{aligned}
& C_{1} \leq u_{n}\left(\beta_{\ell+1} ; \vec{y}\right) \leq C_{2} \\
& C_{1} \leq(-1)^{n} u_{n}\left(\alpha_{1} ; \vec{y}\right) \leq C_{2}
\end{aligned}
$$

Remark. (9.25) says, in the language of [26], that each whole-line $\tilde{J}_{\vec{y}}$ has a regular ground state (see [26, Example 1.5]). It implies critical Lieb-Thirring bounds for perturbations of $\tilde{J}_{\vec{y}}$ in $\left(\beta_{\ell+1}, \infty\right)$. 9.26 implies similar bounds for $\left(-\infty, \alpha_{1}\right)$.

Proof. $B(z) / z$ is positive at $z=0$ and real and nonvanishing on $(-1,1)$, so $B(x)>0$ on $(0,1]$ and $B(x)<0$ on $[-1,0)$. Since $|B( \pm 1)|=1$, we conclude that

$$
B( \pm 1)= \pm 1
$$

Since $u(x ; \vec{y})$ is bounded, strictly positive and continuous in $x$ and $\vec{y}$ for $x \in[-1,1]$ and $\vec{y} \in \mathbb{G},(9.6)$ implies $9.25-(9.26)$. 
$u_{n}^{+}(x)$ is real at $x \in\left\{\alpha_{j}, \beta_{j}\right\}_{j=1}^{\ell+1}$, so $u_{n}^{-}=u_{n}^{+}$and the Jost solutions are not linearly independent. The following gives a second solution which grows linearly in $n$.

Theorem 9.7. Uniformly in $\vec{y} \in \mathbb{G}$ and $z \in \partial \mathcal{F} \cap \mathbb{D}$,

(i)

$$
\left|\frac{\partial u_{n}^{+}(\mathbf{x}(z) ; \vec{y})}{\partial z}\right| \leq C(|n|+1)
$$

$$
\liminf _{n \rightarrow \infty}\left|\frac{1}{n} \frac{\partial u_{n}^{+}(\mathbf{x}(z) ; \vec{y})}{\partial z}\right|>0
$$

(iii) At $\mathbf{x}(z) \in\left\{\alpha_{j}, \beta_{j}\right\}_{j=1}^{\ell+1}$,

$$
v_{n}(\vec{y})=\frac{\partial u_{n}^{+}}{\partial z}(\mathbf{x}(z) ; \vec{y})
$$

is a solution of 8.1, linearly independent of $u_{n}^{+}(\mathbf{x}(z) ; \vec{y})$.

Proof. (i), (ii) By (9.6),

$$
\begin{array}{rl}
\frac{\partial u_{n}^{+}(\mathbf{x}(z) ; \vec{y})}{\partial z}=a_{n}^{-1} & n B^{n-1}(z) B^{\prime}(z) u\left(z ; U^{n}(\vec{y})\right) \\
& +a_{n}^{-1} B^{n}(z) \frac{\partial}{\partial z} u\left(z ; U^{n}(\vec{y})\right)
\end{array}
$$

Since $u\left(z ; U^{n}(\vec{y})\right)$ and $\frac{\partial}{\partial z}\left(u\left(z ; U^{n}(\vec{y})\right)\right.$ are uniformly bounded in $\vec{y}$ and $n$, and $B^{\prime}\left(e^{i \theta}\right)>0$ for all $\theta, 9.28-9.29$ are immediate.

(iii) $u_{n}^{+}(\mathbf{x}(z))$ obeys 8.1 with $z$ replaced by $\mathbf{x}(z)$. Since $\mathbf{x}^{\prime}(z)=0$ at points with $\mathbf{x}(z) \in\left\{\alpha_{j}, \beta_{j}\right\}_{j=1}^{\ell+1}$, we see that $v_{n}$ also solves 8.1. Since $u_{n}^{+}$is bounded and $v_{n}$ is not, they are linearly independent.

Corollary 9.8. For $z \in \mathfrak{e}$, (8.1) has no solution which belongs to $\ell^{2}$ at $+\infty$ or at $-\infty$.

Remark. This result is used in [10].

Proof. If $z \in \mathfrak{e}^{\text {int }}$, this follows from the fact that $\left\|T_{n}(z)^{-1}\right\|$ is bounded, and for $z \in \partial \mathfrak{e}$, it follows from Theorem 9.7.

Corollary 9.9. If $x \in\left\{\alpha_{j}, \beta_{j}\right\}_{j=1}^{\ell+1}$, then

$$
\left\|T_{n}(x)\right\| \leq C(1+|n|)
$$

Proof. Let

$$
\tilde{U}_{n}(x)=\left(\begin{array}{cc}
u_{n+1}^{+}(x) & v_{n+1}(x) \\
a_{n} u_{n}^{+}(x) & a_{n} v_{n}(x)
\end{array}\right)
$$

As in 9.23,

$$
T_{n}(x)=\tilde{U}_{n}(x) U_{0}(x)^{-1}
$$


Since $u^{+}, v$ are independent, $U_{0}$ is invertible and, clearly, $\left\|\tilde{U}_{n}\right\| \leq C(1+$ $|n|)$.

The bound 9.20 diverges as $x$ approaches a point in $\left\{\alpha_{j}, \beta_{j}\right\}_{j=1}^{\ell+1}$. Since $\left\|T_{n}\right\|$ is not bounded at these points, it must. However, we are heading towards a uniform (on $\mathfrak{e}) O(n)$ bound. As a starting point, we need to know more about the right side of (9.14) than the crude bound from (6.6).

Proposition 9.10. For any $\vec{y} \in \mathbb{G}$ and fixed $x_{0} \in\left\{\alpha_{j}, \beta_{j}\right\}_{j=1}^{\ell+1}$,

$$
\lim _{\substack{x \rightarrow x_{0} \\ x \in \mathfrak{e}^{\text {int }}}}\left|x-x_{0}\right|^{-1 / 2}|u(\mathbf{z}(x) ; \vec{y})|^{2} w_{\vec{y}}(x)
$$

exists, is finite and nonvanishing, and is continuous in $\vec{y}$. In (9.35), $\mathbf{z}(x)$ is the point obeying (9.13) with $\mathbf{z} \in \overline{\mathcal{F}} \cap \mathbb{C}_{+}$and $x=\mathbf{x}(\mathbf{z}(x))$.

Remark. This result is subtle because $|u(z ; \vec{y})|^{2}$ can vanish at $x_{0}$. In that case, $w_{\vec{y}}(x)$ has $O\left({\sqrt{\left|x-x_{0}\right|}}^{-1}\right)$ asymptotics rather than $O\left(\sqrt{x-x_{0}}\right)$ asymptotics.

Proof. By the explicit formula for $w_{\vec{y}}(6.10)$ and $(6.22)$, each factor in $w_{\vec{y}}(x)\left|x-x_{0}\right|^{-1 / 2}$ is continuous in $x$ and $\vec{y}$ except for the $\left|x-y_{j}\right|^{-1}$ factor with the $y_{j}$ closest to $x_{0}$. There is a cancelling factor in $|u(z ; \vec{y})|^{2}$, so the limit exists and is continuous in $\vec{y}$.

Theorem 9.11. There is a constant $C$ so that uniformly in $x \in \mathfrak{e}$ and $\vec{y} \in \mathbb{G}$,

$$
\left\|T_{n}(x ; \vec{y})\right\| \leq C(|n|+1)
$$

Proof. For each $x_{0} \in\left\{\alpha_{j}, \beta_{j}\right\}_{j=1}^{\ell+1}$, we prove (9.36) in the half-band starting at $x_{0}$. Form a matrix $\tilde{U}_{n}$ like (9.21) but with $u^{-}$replaced by $\tilde{v}=\left(u^{-}-u^{+}\right) /\left|x-x_{0}\right|^{1 / 2}$. As we have seen, $\tilde{v}$ has a limit as $x$ approaches $x_{0}$ from $\mathfrak{e}^{\text {int }}$. By the Wronskian calculation, $\operatorname{det}\left(U_{n}\right)$ (which is $n$-independent) is bounded as $x$ approaches $x_{0}$.

Finally, writing $\left(u^{-}-u^{+}\right)$as the integral of a derivative and using (9.28), we get

$$
\left|\tilde{U}_{n}\right| \leq C(|n|+1)
$$

SO

$$
\left\|\tilde{U}_{n}\right\| \leq C(|n|+1)
$$

which implies (9.36).

At last, we want to note that (9.6) implies a result about the Jost solutions used in 64]. 
Theorem 9.12. For any compact interval $I \subset \mathfrak{e}^{\text {int }}$, we can write

$$
u_{n}^{+}(x)=e^{i n \theta(x)} f_{n}(x)
$$

where $\theta^{\prime}(x)=\pi \rho_{\mathfrak{e}}(x)$ and $f_{n}$ is real analytic in $x$ with derivatives uniformly bounded in $n$.

Proof. Let $\mathbf{z}(x) \in \overline{\mathcal{F}} \cap \mathbb{C}_{+}$with $\mathbf{x}(\mathbf{z}(x))=x$ and write

$$
B(\mathbf{z}(x))=e^{i \theta(x)}
$$

By $(9.6), u_{n}^{+}$has the form $(9.39)$ and we see that $f_{n}$ has the required properties. By the calculation that led to (4.61), we get the expression for $\theta^{\prime}$.

In [64], this was used to prove that for any $d \mu_{\vec{y}}$, with $\vec{y} \in \mathbb{G}$,

$$
\frac{1}{n} K_{n}(x, x) \rightarrow \frac{\rho_{\mathfrak{e}}(x)}{w_{\vec{y}}(x)}
$$

uniformly on $I$, where $K_{n}$ is the CD kernel (see 62] for definition and background on the classical work of Máté-Nevai-Totik and Totik on limits like (9.40) ). Using the calculations in [5] and identifying $u_{n}^{+}$as a multiple of the Deift-Simon eigenfunctions of [5], one sees that (9.40) implies that

Proposition 9.13. Uniformly on compact subsets of $\mathfrak{e}^{\mathrm{int}} \times \mathbb{G}$,

$$
\frac{1}{n} \sum_{k=0}^{n-1} \operatorname{Re}\left[u_{k}^{+}(x ; \vec{y})^{2}\right] \rightarrow 0 \text { as } n \rightarrow \infty
$$

Remark. That is, $\left(\operatorname{Re} u_{n}^{+}\right)^{2}$ and $\left(\operatorname{Im} u_{n}^{+}\right)^{2}$ have the same average.

\section{Bounds on (Spectral Theorist's) Green's Function}

For $\vec{y} \in \mathbb{G}$, let $J_{\vec{y}}$ be the associated Jacobi matrix and $\tilde{J}_{\vec{y}}$ the associated full-line Jacobi matrix. The (spectral theorist's) Green's functions are defined by

$$
\begin{aligned}
& G_{n m}(z)=\left\langle\delta_{n},\left(J_{\vec{y}}-z\right)^{-1} \delta_{m}\right\rangle \quad n, m=1,2, \ldots \\
& \tilde{G}_{n m}(z)=\left\langle\delta_{n},\left(\tilde{J}_{\vec{y}}-z\right)^{-1} \delta_{m}\right\rangle \quad n, m \in \mathbb{Z}
\end{aligned}
$$

We will use $G_{n m}(z ; \vec{y})$ when we need $\vec{y}$ to be explicit. It is unfortunate that "Green's function" is used both for these objects and for $G_{\mathfrak{e}}(z)$, the potential theorist's Green's function, but both names are ubiquitous. $G_{\mathfrak{e}}$ will appear below in our discussions of $G_{n m}$. 
The analogs of $G_{n m}$ and $\tilde{G}_{n m}$ for $-d^{2} / d x^{2}$ on $L^{2}(0, \infty)$ or $L^{2}(-\infty, \infty)$ are given by

$$
\begin{aligned}
\tilde{G}(x, y ; E) & =\frac{e^{-\kappa|x-y|}}{2 \kappa} \\
G(x, y ; E) & =\frac{e^{-\kappa x>} \sinh \left(\kappa x_{<}\right)}{\kappa}
\end{aligned}
$$

where $E=-\kappa^{2}, x_{>}=\max (x, y), x_{<}=\min (x, y)$. The bounds

$$
\begin{aligned}
& |\tilde{G}(x, y ; E)| \leq(2 \kappa)^{-1} \\
& |G(x, y ; E)| \leq x_{<}
\end{aligned}
$$

play important roles in the analysis of bound states of Schrödinger operators with short-range potentials. Here we find analogs of these bounds for Jacobi matrices in the isospectral torus for $z$ in $\mathbb{R} \backslash \mathfrak{e}$. These bounds were used in [35] to obtain bounds on perturbations of $J_{\vec{y}}$ and $\tilde{J}_{\vec{y}}$.

We need to begin by defining $u_{n}^{ \pm}(x ; \vec{y})$ for $x \in \mathbb{R} \backslash \mathfrak{e}$. Define $\mathbf{z}(x)$ to be the unique point in $\left[\cup_{j=1}^{\ell} C_{j}^{+}\right] \cup(-1,1)$ with

$$
\mathbf{x}(\mathbf{z}(x))=x
$$

Then we define

$$
\begin{aligned}
& u_{n}^{+}(x ; \vec{y})=a_{n}^{-1} B(\mathbf{z}(x))^{n} u\left(\mathbf{z}(x) ; U^{n}(\vec{y})\right) \\
& u_{n}^{-}(x ; \vec{y})=a_{n}^{-1} B(\mathbf{z}(x))^{-n} \overline{u\left(1 / \overline{\mathbf{z}(x)} ; U^{n}(\vec{y})\right)}
\end{aligned}
$$

$u_{n}^{+}$is the analytic continuation of $u_{n}^{+}$as defined for $x \in \mathfrak{e}$ in the last section if we keep $x \in \overline{\mathbb{C}}_{+}$. So is $u_{n}^{-}$since $u_{n}^{-}$is defined on the lower lip of the cuts, so continuing in $\overline{\mathbb{C}}_{+}$brings us to the second sheet and $1 \overline{\mathbf{z}(x)}$. The $-n$ in $u_{n}^{-}$comes from

$$
\overline{B(1 / \overline{\mathbf{z}(x)})}=B(\mathbf{z}(x))^{-1}
$$

Since

$$
|B(\mathbf{z}(x))|=e^{-G_{\mathfrak{e}}(x)}
$$

$u_{n}^{ \pm}$decays exponentially as $n \rightarrow \pm \infty$ and grows exponentially as $n \rightarrow$ $\mp \infty$. It follows that $u_{n}^{ \pm}$must have constant phase (and perhaps we should redefine them to be real). Indeed, the phase is constant on each gap and $u^{+}$and $u^{-}$have opposite phases.

Theorem 10.1. For $x \in \mathbb{R} \backslash \mathfrak{e}$ and $n \leq m$, we have

$$
\tilde{G}_{n m}(x)=\frac{u_{n}^{-}(x) u_{m}^{+}(x)}{\operatorname{Wr}(x)}
$$


where

$$
\mathrm{Wr}(x)=a_{n}\left(u_{n+1}^{+}(x) u_{n}^{-}(x)-u_{n+1}^{-}(x) u_{n}^{+}(x)\right)
$$

Uniformly in $x \in \mathbb{R} \backslash \mathfrak{e}$ and $\vec{y} \in \mathbb{G}$, we have

$$
\left|\tilde{G}_{n m}(x)\right| \leq C e^{-G_{\mathfrak{e}}(x)|n-m|} \operatorname{dist}(x, \mathfrak{e})^{-1 / 2}
$$

Proof. 10.12-110.13) is a standard formula for $\tilde{G}$ in terms of any solutions decaying at $\pm \infty$. As $x$ runs through $\mathbb{R} \backslash \mathfrak{e}, \mathbf{z}(x)$ runs through $\left[\cup_{j=1}^{\ell} C_{j}^{+}\right] \cup(-1,1) \backslash\{0\}$, and $u(\mathbf{z}(x) ; \vec{y})$ is uniformly bounded there. So, by 10.11 , we get 10.14 from

$$
|\operatorname{Wr}(x)| \geq C \operatorname{dist}(x, \mathfrak{e})^{1 / 2}
$$

This is trivial, except near the points $|x|=\infty$ and $x \in\left\{\alpha_{j}, \beta_{j}\right\}_{j=1}^{\ell+1}$ since $\operatorname{Wr}(x)$ is nonvanishing and continuous away from those points.

Since $u$ is regular at $z=0$ and $z=\infty$, the dominant term in 10.8 (10.9) is the $B(z)$ term. In

$$
\operatorname{Wr}(x)=a_{0}\left(u_{1}^{+} u_{0}^{-}-u_{1}^{-} u_{0}^{+}\right)
$$

the dominant term is $B(z)^{-1}$ in $u_{1}^{-}$, so

$$
|\mathrm{Wr}(x)| \sim C|\mathbf{z}(x)|^{-1} \sim C|x| \geq C|x|^{1 / 2}
$$

and (10.15) holds near $|x|=\infty$.

Near points $x_{0} \in\left\{\alpha_{j}, \beta_{j}\right\}_{j=1}^{\ell+1}$, we are looking at the Wronskian of two solutions $u^{+}$and $u^{-}$which approach each other. Thus, we get a Wronskian which goes to zero as $\left(\mathbf{z}(x)-\mathbf{z}\left(x_{0}\right)\right)$ times the Wronskian of $u^{+}$and $d u^{-} / d z$. We have already seen in the last section that these are two linearly independent solutions, so their Wronskian is nonzero and thus, near $x_{0} \in\left\{\alpha_{j}, \beta_{j}\right\}_{j=1}^{\ell+1}$, for some $C>0$,

$$
\operatorname{Wr}(x) \geq C\left|\mathbf{z}(x)-\mathbf{z}\left(x_{0}\right)\right|=C \cdot O\left(\left|x-x_{0}\right|^{1 / 2}\right)
$$

proving 10.15.

As $x$ approaches a point $x_{0} \in\left\{\alpha_{j}, \beta_{j}\right\}_{j=1}^{\ell+1}$ from $\mathbb{R} \backslash \mathfrak{e}$, typically (i.e., except for special values of $\vec{y}, n$, and $m), \tilde{G}_{n m}(x) \rightarrow \infty$. For $G_{n m}(x)$, this is normally not true, which is why one expects bounds in this case that are not divergent at $x_{0}$. However, there are special values of $\vec{y}$ for which this is not the case. One sees this for $n=m=1$ since

$$
G_{11}(z)=m(z)
$$

the $m$-function of $(6.3) . m$ is meromorphic on $\mathcal{S}$, so it normally has a finite value at $x_{0}$ but might have a pole there. 
Definition. Fix $\vec{y} \in \mathbb{G}$. A point $x_{0} \in\left\{\alpha_{j}, \beta_{j}\right\}_{j=1}^{\ell+1}$ is said to be a resonance if and only if it is a pole of $m_{\vec{y}}(z)$ (in the sense of poles on $\mathcal{S}$ which means $\left(x-x_{0}\right)^{-1 / 2}$ divergence since $x_{0}$ is a branch point). Otherwise, we say $x_{0}$ is nonresonant.

It is easy to see that resonances are equivalent to $u_{0}^{+}\left(x_{0}\right)=0$.

Theorem 10.2. Fix $\vec{y} \in \mathbb{G}$. For $n, m \geq 1$ and $x \in \mathbb{R} \backslash \mathfrak{e}$, we have

$$
G_{n m}(x)=\tilde{G}_{n m}(x)-\tilde{G}_{0 n}(x) \tilde{G}_{0 m}(x) \tilde{G}_{00}(x)^{-1}
$$

Suppose $x_{0}$ is nonresonant for $\vec{y}$. Let $I$ be the open interval with one end at $x_{0}$ and the other at the middle of the gap if $x_{0}$ is a boundary point of a finite gap and $I \subset \mathbb{R} \backslash \mathfrak{e}$ with $|I|=1$ if $x_{0} \in\left\{\alpha_{1}, \beta_{\ell+1}\right\}$. Then for all $n, m \geq 1$ and $x \in I$, we have

$$
\begin{aligned}
& \left|G_{n m}(x)\right| \leq C \min (n, m) \\
& \left|G_{n m}(x)\right| \leq C\left|x-x_{0}\right|^{-1 / 2}
\end{aligned}
$$

for some constant $C$.

Proof. Since, for $x \in \mathbb{R} \backslash \mathfrak{e}$ fixed,

$$
u_{0}^{+} u_{n}^{-}-u_{0}^{-} u_{n}^{+} \equiv q_{n}
$$

vanishes at $n=0, q_{n}(x)=C(x) p_{n}(x)$ for some constant (depending on $x)$. Thus, by the standard formula for $G_{n m}$,

$$
G_{n m}=\frac{p_{n} u_{m}^{+}}{\operatorname{Wr}\left(u^{+}, p\right)}, \quad 1 \leq n \leq m
$$

we get

$$
G_{n m}(x)=q_{n}(x) u_{m}^{+}(x) \widetilde{\operatorname{Wr}}(x)^{-1}
$$

where $\widetilde{\mathrm{Wr}}$ is the Wronskian of $u^{+}$and $q$.

By 10.22,

$$
\widetilde{\mathrm{Wr}}(x)=u_{0}^{+}(x) \mathrm{Wr}(x)
$$

so 10.24 becomes

$$
G_{n m}(x)=\frac{u_{0}^{+}(x) u_{n}^{-}(x) u_{m}^{+}(x)-u_{0}^{-}(x) u_{n}^{+}(x) u_{m}^{+}(x)}{u_{0}^{+}(x) \operatorname{Wr}(x)}
$$

The first term in 10.26 is, by $10.12, \tilde{G}_{n m}(x)$. If we note that (also by (10.12)

$$
\tilde{G}_{0 n}(x) \tilde{G}_{0 m}(x) \tilde{G}_{00}(x)^{-1}=\frac{u_{0}^{-}(x) u_{n}^{+}(x) u_{m}^{+}(x)}{u_{0}^{+}(x) \operatorname{Wr}(x)}
$$

we see that the second term in 10.26 is the second term in 10.19, so we have proven (10.19. 
As we noted above, $x_{0}$ nonresonant implies that $u_{0}^{+}\left(x_{0}\right) \neq 0$. Thus, (10.26) shows that

$$
\sup _{\substack{n, m \geq 1 \\ x \in I}}\left|G_{n m}(x)\right| \leq C \operatorname{Wr}(x)^{-1}
$$

which, by (10.16), proves 10.21).

We claim first that (10.20) is implied by

$$
\left|q_{n}(x) B(\mathbf{z}(x))^{n}\right| \leq C n\left|x-x_{0}\right|^{1 / 2}
$$

For, by 10.26 and 10.16),

$\left|G_{n m}(x)\right| \leq C\left|u_{m}^{+}(x) B(\mathbf{z}(x))^{-m}\right||B(\mathbf{z}(x))|^{m-n}\left|q_{n}(x) B(\mathbf{z}(x))^{n}\right|\left|x-x_{0}\right|^{-1 / 2}$

so 10.29 together with $|B(\mathbf{z}(x))| \leq 1$ and the fact that $\left|u_{m}^{+}(x) B(\mathbf{z}(x))^{-m}\right|$ is bounded implies 10.20 .

Next, note that because of the definitions 110.8$)-(10.9)$ and the constancy of the phase of $u_{n}^{ \pm}$, we see that for all $n$,

$$
q_{n}\left(x_{0}\right)=0
$$

Define

$$
h_{n}(z)=q_{n}(\mathbf{x}(z)) B(z)^{n}
$$

By (10.31) and $\left|\mathbf{z}(x)-\mathbf{z}\left(x_{0}\right)\right|=O\left(\left|x-x_{0}\right|^{1 / 2}\right)$, (10.29) follows from

$$
\sup _{z \in \mathbf{z}(I)}\left|\frac{d h_{n}(z)}{d z}\right| \leq C n
$$

$h_{n}$ is built out of $u$ 's which have bounded derivatives and $B(z)^{n}$ which has a derivative bounded by $C n$, so 10.33 holds.

\section{REFERENCES}

[1] L. V. Ahlfors, Complex Analysis. An Introduction to the Theory of Analytic Functions of One Complex Variable, McGraw-Hill, New York, 1978.

[2] L. Ahlfors and L. Bers, Riemann's mapping theorem for variable metrics Ann. of Math. (2) $\mathbf{7 2}$ (1960), 385-404.

[3] A. I. Aptekarev, Asymptotic properties of polynomials orthogonal on a system of contours, and periodic motions of Toda chains, Math. USSR Sb. $\mathbf{5 3}$ (1986), 233-260; Russian original in Mat. Sb. (N.S.) 125(167) (1984), 231258. Math. USSR-Sb. 53 (1986), 233-260.

[4] M. A. Armstrong, Basic Topology, corrected reprint of the 1979 original, Undergraduate Texts in Mathematics, Springer-Verlag, New York-Berlin, 1983.

[5] A. Avila, Y. Last, and B. Simon, Bulk universality and clock spacing of zeros for ergodic Jacobi matrices with a.c. spectra, preprint.

[6] D. Barrios Rolanía and G. López Lagomasino, Ratio asymptotics for polynomials orthogonal on arcs of the unit circle, Constr. Approx. 15 (1999), 1-31. 
[7] V. Batchenko and F. Gesztesy, On the spectrum of Jacobi operators with quasi-periodic algebro-geometric coefficients, Int. Math. Res. Papers No. 10, $511-563(2005)$.

[8] A. F. Beardon, Inequalities for certain Fuchsian groups, Acta Math. 127 (1971), 221-258.

[9] M. Bello Hernández and G. López Lagomasino, Ratio and relative asymptotics of polynomials orthogonal on an arc of the unit circle, J. Approx. Theory 92 (1998), 216-244.

[10] J. Breuer, Y. Last, and B. Simon, The Nevai condition, preprint.

[11] W. Bulla, F. Gesztesy, H. Holden, and G. Teschl, Algebro-geometric quasiperiodic finite-gap solutions of the Toda and Kac-van Moerbeke hierarchies, Memoirs Amer. Math. Soc. 135, No. 641 (1998)

[12] W. Burnside On a class of automorphic functions, Proc. London Math. Soc. 23 (1891), 49-88

[13] W. Burnside Further note on automorphic functions, Proc. London Math. Soc. 23 (1891), 281-295.

[14] J. Christiansen, B. Simon, and M. Zinchenko, Finite gap Jacobi matrices: An announcement, to appear in Proc. 2007 OPSFA, Marseille.

[15] J. Christiansen, B. Simon, and M. Zinchenko, Finite gap Jacobi matrices, II. The Szegö class, in preparation.

[16] J. Christiansen, B. Simon, and M. Zinchenko, Finite gap Jacobi matrices, III. Beyond the Szegö class, in preparation.

[17] W. Craig, The trace formula for Schrödinger operators on the line, Comm. Math. Phys. 126 (1989), 379-407.

[18] D. Damanik, R. Killip, and B. Simon, Perturbations of orthogonal polynomials with periodic recursion coefficients, preprint.

[19] D. Damanik and B. Simon, Jost functions and Jost solutions for Jacobi matrices, I. A necessary and sufficient condition for Szegö asymptotics, Invent. Math. 165 (2006), 1-50.

[20] S. A. Denisov, On Rakhmanov's theorem for Jacobi matrices, Proc. Amer. Math. Soc. 132 (2004), 847-852.

[21] B. A. Dubrovin, V. B. Matveev, and S. P. Novikov, Nonlinear equations of Korteweg-de Vries type, finite-band linear operators and Abelian varieties, Uspekhi Mat. Nauk 31 (1976), no. 1(187), 55-136. [Russian]

[22] P. Erdös and P. Turán, On interpolation. III. Interpolatory theory of polynomials, Annals of Math. (2) 41 (1940), 510-553.

[23] H. M. Farkas and I. Kra, Riemann Surfaces, Graduate Texts in Mathematics, 71, Springer, New York-Berlin, 1980.

[24] H. Flaschka and D. W. McLaughlin, Canonically conjugate variables for the Korteweg-de Vries equation and the Toda lattice with periodic boundary conditions, Progr. Theoret. Phys. 55 (1976), 438-456.

[25] I. Fonseca and W. Gangbo, Degree Theory in Analysis and Applications, Oxford Lecture Series in Mathematics and Its Applications, 2, The Clarendon Press, Oxford Univ. Press, New York, 1995.

[26] R. Frank, B. Simon, and T. Weidl, Eigenvalue bounds for perturbations of Schrödinger operators and Jacobi matrices with regular ground states, to appear in Comm. Math. Phys.

[27] G. Freud, Orthogonal Polynomials, Pergamon Press, Oxford-New York, 1971. 
[28] F. Gesztesy and H. Holden, Soliton Equations and Their Algebro-Geometric Solutions. Vol. I: (1+1)-Dimensional Continuous Models, Cambridge Studies in Advanced Mathematics, 79, Cambridge University Press, 2003.

[29] F. Gesztesy, H. Holden, J. Michor, and G. Teschl, Soliton Equations and Their Algebro-Geometric Solutions. Vol. II: $(1+1)$-Dimensional Discrete Models, Cambridge Studies in Advanced Mathematics, 114, Cambridge University Press, Cambridge, 2008.

[30] P. Griffiths and J. Harris, Principles of Algebraic Geometry, John Wiley \& Sons, New York, 1978.

[31] V. Guillemin and A. Pollack, Differential Topology, Prentice-Hall, Englewood Cliffs, N.J., 1974.

[32] A. Hatcher, Algebraic Topology, Cambridge University Press, Cambridge, 2002.

[33] D. A. Hejhal, Universal covering maps for variable regions, Math. Z. 137 (1974), 7-20.

[34] L. L. Helms, Introduction to Potential Theory, Pure and Applied Mathematics, 22, Wiley-Interscience, New York, 1969.

[35] D. Hundertmark and B. Simon, Eigenvalue bounds in the gaps of Schrödinger operators and Jacobi matrices, J. Math. Anal. Appl. 340 (2008), 892-900.

[36] R. Killip and B. Simon, Sum rules for Jacobi matrices and their applications to spectral theory, Ann. of Math. 158 (2003), 253-321.

[37] S. Katok, Fuchsian Groups, University of Chicago Press, Chicago, 1992.

[38] W. Krawcewicz and J. Wu, Theory of Degrees With Applications to Bifurcations and Differential Equations, Canadian Mathematical Society Series of Monographs and Advanced Texts, John Wiley \& Sons, New York, 1997.

[39] I. M. Krichever, Algebraic curves and nonlinear difference equations, Uspekhi Mat. Nauk 33 (1978), no. 4(202), 215-216. [Russian]

[40] N. S. Landkof, Foundations of Modern Potential Theory, Springer-Verlag, Berlin-New York, 1972.

[41] Y. Last and B. Simon, The essential spectrum of Schrödinger, Jacobi, and CMV operators, J. Anal. Math. 98 (2006), 183-220.

[42] N. G. Lloyd, Degree Theory, Cambridge Tracts in Mathematics, 73, Cambridge University Press, Cambridge-New York-Melbourne, 1978.

[43] A. Martínez-Finkelshtein, Equilibrium problems of potential theory in the complex plane, in "Orthogonal Polynomials and Special Functions," pp. 79117, Lecture Notes in Mathematics, 1883, Springer, Berlin, 2006.

[44] H. P. McKean and P. van Moerbeke, The spectrum of Hill's equation, Invent. Math. 30 (1975), 217-274.

[45] J. W. Milnor, Topology From the Differentiable Viewpoint, revised reprint of the 1965 original, Princeton Landmarks in Mathematics, Princeton University Press, Princeton, N.J., 1997.

[46] R. Miranda, Algebraic Curves and Riemann Surfaces, Graduate Studies in Mathematics, 5, American Mathematical Society, Providence, R.I., 1995.

[47] P. Nevai, Orthogonal polynomials, Mem. Amer. Math. Soc. 18 (1979), no. 213, $185 \mathrm{pp}$.

[48] S. J. Patterson, The limit set of a Fuchsian group, Acta Math. 136 (1976), 241-273. 
[49] F. Peherstorfer and P. Yuditskii, Asymptotics of orthonormal polynomials in the presence of a denumerable set of mass points, Proc. Amer. Math. Soc. 129 (2001), 3213-3220.

[50] F. Peherstorfer and P. Yuditskii, Asymptotic behavior of polynomials orthonormal on a homogeneous set, J. Anal. Math. 89 (2003), 113-154.

[51] F. Peherstorfer and P. Yuditskii, Remark on the paper "Asymptotic behavior of polynomials orthonormal on a homogeneous set", arXiv math.SP/0611856.

[52] H. Poincaré, Mémoire sur les fonctions fuchsiennes, Acta Math. 1 (1882), 193-294.

[53] T. Radó, Über die Fundamentalabbildungen schlichter Gebiete, Acta Litt. ac. Scient. Univ. Hung. 1 (1923), 240-251.

[54] T. Ransford, Potential Theory in the Complex Plane, Press Syndicate of the University of Cambridge, New York, 1995.

[55] C. Remling, The absolutely continuous spectrum of Jacobi matrices, preprint.

[56] W. Rudin, Real and Complex Analysis, 3rd edition, McGraw-Hill, New York, 1987.

[57] B. Simon, A canonical factorization for meromorphic Herglotz functions on the unit disk and sum rules for Jacobi matrices, J. Funct. Anal. 214 (2004), 396-409.

[58] B. Simon, Ratio asymptotics and weak asymptotic measures for orthogonal polynomials on the real line, J. Approx. Theory 126 (2004), 198-217.

[59] B. Simon, Orthogonal Polynomials on the Unit Circle, Part 1: Classical Theory, AMS Colloquium Publications 54.1, American Mathematical Society, Providence, R.I., 2005.

[60] B. Simon, Orthogonal Polynomials on the Unit Circle, Part 2: Spectral Theory, AMS Colloquium Publications 54.2, American Mathematical Society, Providence, R.I., 2005.

[61] B. Simon, Equilibrium measures and capacities in spectral theory, Inverse Problems and Imaging 1 (2007), 713-772.

[62] B. Simon, The Christoffel-Darboux kernel, to appear in "Perspectives in PDE, Harmonic Analysis and Applications" in honor of V. G. Maz'ya's 70th birthday, to be published in Proceedings of Symposia in Pure Mathematics.

[63] B. Simon, Regularity and the Cesàro-Nevai class, to appear in J. Approx. Theory.

[64] B. Simon, Two extensions of Lubinsky's universality theorem, to appear in J. Anal. Math.

[65] B. Simon, Szegö's Theorem and Its Descendants: Spectral Theory for L2 Perturbations of Orthogonal Polynomials, in preparation; to be published by Princeton University Press.

[66] M. Sodin and P. Yuditskii, Almost periodic Jacobi matrices with homogeneous spectrum, infinite-dimensional Jacobi inversion, and Hardy spaces of character-automorphic functions, J. Geom. Anal. 7 (1997), 387-435.

[67] M. Spivak, A Comprehensive Introduction to Differential Geometry, Volume I, 2nd ed., Publish or Perish, Wilmington, Del., 1979.

[68] H. Stahl and V. Totik, General Orthogonal Polynomials, in "Encyclopedia of Mathematics and its Applications," 43, Cambridge University Press, Cambridge, 1992. 
[69] D. Sullivan, The density at infinity of a discrete group of hyperbolic motions, Inst. Hautes Études Sci. Publ. Math. 50 (1979), 171-202.

[70] G. Szegö, Orthogonal Polynomials, Amer. Math. Soc. Colloq. Publ. 23, American Mathematical Society, Providence, R.I., 1939; third ed., 1967.

[71] G. Teschl, Jacobi Operators and Completely Integrable Nonlinear Lattices, Math. Surv. Monographs, 72, American Mathematical Society, Providence, R.I., 2000.

[72] V. Totik, Polynomial inverse images and polynomial inequalities, Acta Math. 187 (2001), 139-160.

[73] M. Tsuji, Potential Theory in Modern Function Theory, reprinting of the 1959 original, Chelsea, New York, 1975.

[74] J. L. Ullman, On the regular behaviour of orthogonal polynomials, Proc. London Math. Soc. (3) 24 (1972), 119-148.

[75] J. L. Ullman, Orthogonal polynomials for general measures. I, in "Rational Approximation and Interpolation" (Tampa, FL, 1983), pp. 524-528, Lecture Notes in Mathematics, 1105, Springer, Berlin, 1984.

[76] J. L. Ullman, Orthogonal polynomials for general measures. II, in "Orthogonal Polynomials and Applications" (Bar-le-Duc, 1984), pp. 247-254, Lecture Notes in Mathematics, 1171, Springer, Berlin, 1985.

[77] W. Van Assche, Invariant zero behaviour for orthogonal polynomials on compact sets of the real line, Bull. Soc. Math. Belg. Ser. B 38 (1986), 1-13.

[78] P. van Moerbeke, The spectrum of Jacobi matrices, Invent. Math. 37 (1976), $45-81$.

[79] V. A. Vassiliev, Introduction to Topology, Student Mathematical Library, 14, American Mathematical Society, Providence, R.I., 2001.

[80] H. Widom, Polynomials associated with measures in the complex plane, J. Math. Mech. 16 (1967), 997-1013.

[81] H. Widom, Extremal polynomials associated with a system of curves in the complex plane, Adv. in Math. 3 (1969), 127-232. 